\title{
High-Energy Atmospheric Physics: Terrestrial Gamma-Ray Flashes and Related Phenomena
}

\author{
Joseph R. Dwyer · David M. Smith • Steven A. Cummer
}

Received: 1 December 2011 / Accepted: 4 May 2012 / Published online: 3 June 2012

(C) The Author(s) 2012. This article is published with open access at Springerlink.com

\begin{abstract}
It is now well established that both thunderclouds and lightning routinely emit $\mathrm{x}$-rays and gamma-rays. These emissions appear over wide timescales, ranging from submicrosecond bursts of x-rays associated with lightning leaders, to sub-millisecond bursts of gamma-rays seen in space called terrestrial gamma-ray flashes, to minute long glows from thunderclouds seen on the ground and in or near the cloud by aircraft and balloons. In particular, terrestrial gamma-ray flashes (TGFs), which are thought to be emitted by thunderclouds, are so bright that they sometimes saturate detectors on spacecraft hundreds of kilometers away. These TGFs also generate energetic secondary electrons and positrons that are detected by spacecraft in the inner magnetosphere. It is generally believed that these $\mathrm{x}$-ray and gamma-ray emissions are generated, via bremsstrahlung, by energetic runaway electrons that are accelerated by electric fields in the atmosphere. In this paper, we review this newly emerging field of High-Energy Atmospheric Physics, including the production of runaway electrons, the production and propagation of energetic radiation, and the effects of both on atmospheric electrodynamics.
\end{abstract}

Keywords Thunderstorms · Lightning · x-rays · Gamma-rays · Energetic radiation · Atmospheric electricity

\footnotetext{
J.R. Dwyer $(\bowtie)$

Department of Physics and Space Sciences, Florida Institute of Technology, Melbourne, FL 32901, USA

e-mail: jdwyer@fit.edu

D.M. Smith

Physics Department and Santa Cruz Institute for Particle Physics, University of California, Santa Cruz, CA 95064, USA

e-mail: dsmith8@ucsc.edu
}

S.A. Cummer

Electrical and Computer Engineering Department, Duke University, Durham, NC 27708, USA

e-mail: cummer@ee.duke.edu 


\section{Introduction}

Despite the ubiquity of thunderstorms, lightning, and related electrical phenomena, many important electromagnetic processes in our atmosphere are poorly understood. For example, many questions remain about thundercloud electrification and discharge mechanisms, lightning initiation, propagation and attachment processes, compact intra-cloud discharges, the global electrical circuit, and transient luminous events (Rakov and Uman 2003). Traditionally, these topics have been studied using classical electromagnetism. However, in the last few years, a growing body of literature has emerged that describes the production, transport and interactions of energetic particles in our atmosphere. Specifically, it is now well established that thunderclouds, lightning, and long laboratory sparks in air all produce energetic runaway electrons and accompanying $\mathrm{x}$-ray and gamma-ray emissions. Terrestrial gamma-ray flashes (TGFs), bright bursts of multi-MeV gamma-rays that are seen hundreds of kilometers away by spacecraft, are particularly impressive examples of runaway electron production in our atmosphere. Moreover, such high-energy particles interact with air atoms, forming low-energy electron and ion populations that may greatly increase the conductivity of air, potentially affecting the physics of thunderclouds and lightning. We shall refer to the rapidly expanding field of energetic particle and radiation physics in terrestrial and planetary atmospheres, and their effects, as High-Energy Atmospheric Physics. Not only does this field impact traditional atmospheric electricity and lightning physics, it also has implications for the study of cosmic-ray extensive air showers, discharge physics, space physics, plasma physics, and aviation safety.

In this paper, we shall review runaway electron production mechanisms, including relativistic runaway electron avalanches, thermal runaway electron production and the relativistic feedback mechanism, bremsstrahlung emissions, and x-ray and gamma-ray propagation. We shall also review energetic radiation observations, including terrestrial gammaray flashes (TGFs) and terrestrial electron beams (TEBs), gamma-ray glows from thunderclouds, neutron observations, x-rays from lightning and laboratory sparks, and radio frequency emissions and observations. Finally, we shall discuss recent modeling work and implications for atmospheric phenomena such as sprites and other transient luminous events (TLEs), compact intra-cloud discharges (CIDs), thunderstorm electrification and lightning initiation, and radiation doses to individuals in aircraft.

\section{High-Energy Atmospheric Physics Theory}

\subsection{Wilson Runaway Electrons}

In 1925, C.T.R. Wilson discovered the runaway electron mechanism in which fast electrons may obtain large energies from static electric fields in air (Wilson 1925). Specifically, when the rate of energy gain from an electric field exceeds the rate of energy loss from interactions with air then the energy of an electron will increase and it will "run away." Runaway electrons are produced in electric fields greater than the so-called break-even field, $E_{b}=2.18 \times 10^{5} \mathrm{~V} / \mathrm{m} \times n$, corresponding to the rate that minimum ionizing electrons lose energy, where $n$ is the density of air with respect to that at sea level. Simulations have shown that due to elastic scattering, the field required for runaway electrons to propagate large distances is actually about $30 \%$ higher than $E_{b}$ (Dwyer 2003). This field is about a factor of ten below the conventional breakdown field and is comparable to maximum fields seen inside thunderclouds (Rakov and Uman 2003). Indeed, Marshall et al. (1995) found that 


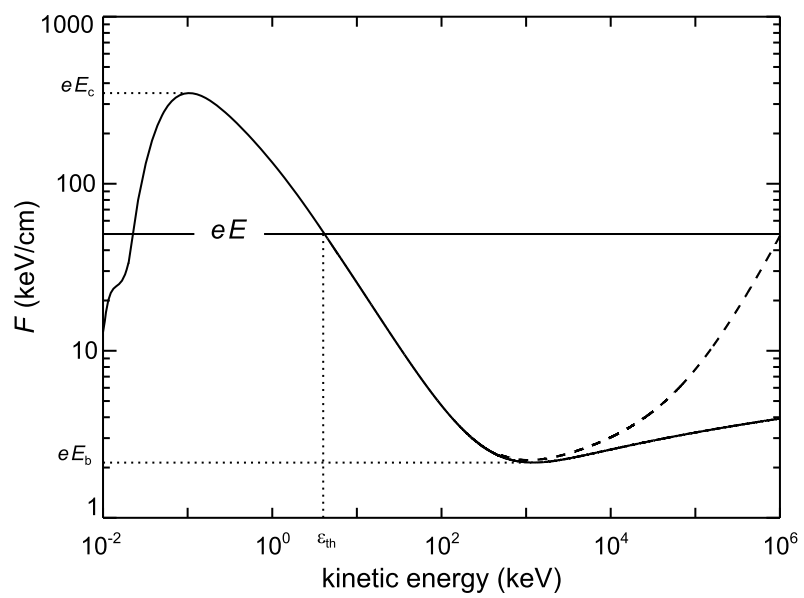

Fig. 1 The effective frictional force experienced by a free electron (or positron) moving through air at STP as a function of kinetic energy. The solid curve is due to inelastic scattering of the electron by air molecules, and the dashed curve indicates the effects of bremsstrahlung emission. The horizontal line shows the electric force from a $5.0 \times 10^{6} \mathrm{~V} / \mathrm{m}$ electric field. Runaway electrons occur for kinetic energies greater than the threshold energy, $\varepsilon>\varepsilon_{t h}$. In the figure, $E_{c}$ is the critical electric field strength for which low-energy thermal electrons will run away, and $E_{b}$ is the so-called break-even field

balloon soundings inside thunderclouds often measured maximum electric fields near the break-even field, suggesting a possible connection between lightning initiation and runaway election production (also see Stolzenburg et al. 2007).

Figure 1 shows the rate of energy loss of an energetic electron moving in air (effective frictional force). The plot also shows that rate of energy gain from a strong electric field (horizontal line). As can be seen, in order for an electron to run away, it must have an initial kinetic energy above the threshold, $\varepsilon_{t h}$. Such energetic "seed" electron, with energies above $\varepsilon_{t h}$, may be provided from an external source such as cosmic-rays or radioactive decays. Note that the kinetic energy, $\varepsilon_{t h}$, required for the seed particles decreases rapidly with increasing electric field. When the electric field is increased above the critical field, $E_{c}$, above the energy loss curve for all kinetic energies, then all free electrons may run away, and, in particular, the thermal population created at low energies may run away. This mechanism is usually called "cold runaway" or "thermal runaway," and does not require any external seed particles (Gurevich 1961).

According to Wilson's original work, the energetic seeds that result in runaway electrons are all provided by external sources such as cosmic-rays (or radioactive decays). Therefore, for each atmospheric cosmic-ray secondary electron that arrives, at most one runaway electron may be generated. More concisely, each atmospheric cosmic-ray secondary electron may become a runaway electron in a sufficiently large electric field. Although a runaway electron may gain energy and travel farther than the seed particle without an electric field, this mechanism will not produce large fluxes of runaway electrons (McCarthy and Parks 1992), especially the large fluxes known to be associated with terrestrial gamma-ray flashes (TGFs). On the other hand, it is possible for thermal runaway to provide the energetic seeds, which subsequently experience additional energy gain and avalanche multiplication. This combination could potentially explain TGFs, as will be discussed below. 


\subsection{Relativistic Runaway Electron Avalanches (RREAs)}

\subsubsection{Introduction}

In 1992, Gurevich, Milikh and Roussel-Dupré showed that when Møller scattering (electronelectron elastic scattering) is included, the runaway electrons described by Wilson will undergo avalanche multiplication, resulting in a large number of relativistic runaway electrons for each energetic seed electron injected into the high-field region (Gurevich et al. 1992; Gurevich and Zybin 2001). This avalanche mechanism is commonly referred to as the Relativistic Runaway Electron Avalanche (RREA) mechanism (Babich et al. 1998, 2001a). Although Wilson appears to have been aware of the runaway electron avalanche multiplication (Williams 2010), referring to it as a "snowball effect," he did not provide quantitative calculations of the avalanche properties. The runaway electron avalanche threshold electric field was estimated by Symbalisty et al. (1998) to be in the range $2.83-3.05 \times 10^{5} \mathrm{~V} / \mathrm{m} \times n$, where $n$ is the density of air with respect to that at sea level. Dwyer (2003) investigated the avalanche threshold in detail and found it to be

$$
E_{t h}=2.84 \times 10^{5} \mathrm{~V} / \mathrm{m} \times n,
$$

in agreement with the value $2.83 \times 10^{5} \mathrm{~V} / \mathrm{m} \times n$ by Babich et al. (2004a). This threshold field is slightly larger than the break-even field, which is the minimum value of the ionization energy loss curve seen in curve in Fig. 1. If the runaway electrons traveled exactly along the electric field lines, then this would be the threshold for runaway electrons propagation and avalanche multiplication. However, elastic scattering of the electrons with atomic nuclei (Coulomb scattering) and the atomic electrons (Møller scattering) causes deviations in the electron trajectories. In addition, secondary electrons from Møller scattering are usually not created along the field line. As a result, about $30 \%$ larger electric fields are required in order for the electrons to run away and avalanche multiply. The runaway electron avalanche threshold is near the maximum electric field strength measured inside thunderclouds (Rakov and Uman 2003), suggesting that runaway electron avalanches may be common inside thunderclouds.

Following Gurevich et al. (1992), a series of papers by several groups developed the underlying physics involved in RREAs: Roussel-Dupré et al. (1994) first derived the kinetic equation (Boltzmann equation) for relativistic runaway electron avalanches. Symbalisty et al. (1998) also investigated runaway electron avalanches using the kinetic equation with the ionization integral derived by Gurevich et al. (1998). In their paper, they pointed out that the numerical solutions of Roussel-Dupré et al. (1994) suffered from a numerical instability and so were not valid. Lehtinen et al. (1999) found substantial disagreement between their avalanche rates and those of Symbalisty et al. (1998), and attributed this difference to the formulation of the ionization process used by Symbalisty et al. (1998). The work by Symbalisty et al. (1998) was superseded by the work of Babich et al. (2001a), who improved the formulation of the ionization processes, bringing the avalanche rates into better agreement with the Lehtinen et al. (1999) results, although the avalanche rates found by the more sophisticated ELIZA Monte Carlo code still disagreed. This discrepancy was later addressed by Babich et al. (2004a). Gurevich et al. (1997) and Gurevich and Milikh (1999) modeled x-ray emissions from RREAs, and Gurevich et al. (2000) considered pair production. Gurevich et al. (2001b) developed the kinetic theory for inhomogeneous electric fields. Babich (2004) derived the collision operator for relativistic electrons. Finally, Babich et al. (2007b) and Carlson et al. (2008) investigated the seeding process by atmospheric cosmic-rays. 


\subsubsection{RREA Simulation Techniques}

In this review, we shall compare recent results from four groups, including three Monte Carlo codes and one Boltzmann equation code: The first paper to accurately calculate avalanche rates and the runaway electron energy spectrum was Lehtinen et al. (1999). They developed a Monte Carlo simulation that calculated the propagation of energetic electrons in electric and magnetic fields and included energy losses from ionization and atomic excitation, Møller scattering and angular diffusion from elastic scattering with atomic nuclei. The code did not include bremsstrahlung production and energy losses.

Following the approach of Lehtinen et al. (1999), Dwyer (2003) developed a Monte Carlo simulation called REAM (Runaway Electron Avalanche Model) that included, in an accurate form, all the important interactions involving runaway electrons, including energy losses through ionization and atomic excitation and Møller scattering (also see Dwyer 2007). Unlike the earlier work, however, this simulation fully models elastic scattering using a shielded-Coulomb potential, rather than relying on a diffusion approximation, and also includes bremsstrahlung production of $\mathrm{x}$-rays and gamma-rays and the subsequent propagation of the photons, including photoelectric absorption, Compton scattering, pair production and Rayleigh scattering. In addition, new features included the incorporation of positron propagation, annihilation, bremsstrahlung production and the generation of energetic seed electrons via Bhabha scattering of positrons and via Compton scattering and photoelectric absorption of energetic photons.

An independent Monte Carlo code, the VNIIEF code ELIZA (Babich et al. 2001a, 2004a, 2005), includes the following elementary processes: for photons, the code includes Compton scattering with allowance for bound electrons, Rayleigh scattering, photo absorption with emission of fluorescent photons and Auger electrons, and production of electron-positron pairs and triplets. For electrons, the code includes elastic scattering by atomic nuclei, ionization and excitation of atomic electron shells, and bremsstrahlung. For positrons, it includes elastic scattering by nuclei, scattering by free electrons, bremsstrahlung and two body annihilation.

Roussel-Dupré et al. (2008) compiled accurate energy loss rates and interaction crosssections and further developed the Fokker-Planck form of the relativistic Boltzmann equation for RREAs. They then solved the equations numerically using a finite volume, cell centered, time explicit, spatially second order accurate algorithm.

Most recently, Celestin and Pasko (2010) developed a Monte Carlo code that includes the relativistic binary-encounter-Bethe (RBEB) electron impact model to study RREAs. The RBEB differential ionization cross-sections give slightly different avalanche rates than the Møller scattering cross-sections commonly used in other work. Their Monte Carlo closely followed the work by Lehtinen et al. (1999) and in particular included an angular diffusion approximation, rather than fully modeling the elastic scattering as in the REAM Monte Carlo code. Their code also did not simulate the photons or positrons. The photons, in particular, play an important role in the avalanche development at low fields and so care should be taken when comparing simulations that include bremsstrahlung production and photons propagation (e.g., ELIZA and REAM) and codes that do not such as that of Celestin and Pasko (2010) and Lehtinen et al. (1999), especially at very low field values near the avalanche threshold.

In principle, the widely available code GEANT4 (Agostinelli et al. 2003) could perform similar calculations, although to our knowledge no such work has been published with the exception of Carlson et al. (2007) and Carlson (2009). 


\subsubsection{Avalanche Length Comparison}

Figure 2 shows a plot of the avalanche (e-folding) length, $\lambda$, as a function of electric field strength at sea level as calculated by the four codes discussed above. As can be seen, all the results are in good agreement over a wide range of electric field strengths. To calculate the avalanche length at other altitudes, the electric field on the horizontal axis should be multiplied by $n$ and the avalanche length on the vertical axis should be divided by $n$, where $n$ is the density of air relative to that at sea level. In Fig. 2, we show Monte Carlo calculations by Lehtinen et al. (1999) with an empirical fit by Inan and Lehtinen (2005); Monte Carlo calculations presented by Babich et al. (2004a, 2005) with an empirical fit to the same Monte Carlo results by Babich et al. (2004a); Monte Carlo calculations by Dwyer (2003) and Coleman and Dwyer (2006) with an empirical fit given by Coleman and Dwyer (2006); Boltzmann equation calculations by Roussel-Dupré et al. (2008) with an empirical fit by Milikh and Roussel-Dupré (2010); and Monte Carlo calculations by Celestin and Pasko (2010) for the Møller scattering cross-section and an alternative electron impact ionization model for the secondary electron production. Because some work found the avalanche lengths $(\lambda)$ directly and some work found the avalanche times $(\tau)$, we convert all data to avalanche lengths using the work of Coleman and Dwyer (2006) as follows $\lambda=\tau v$, where $v=0.89 c$ is the average avalanche propagation speed, which is in good agreement with the speed found by Babich and Bochkov (2011).

We next consider a simple description of the runaway electrons in an avalanche. Let $F_{o}$ be the flux of external energetic seed particles that run away, e.g., the flux due to atmospheric cosmic-ray particles and radioactive decays. Depending upon the altitude and the geographic location, $F_{o}$ is in the range $100-10,000 \mathrm{~m}^{-2} \mathrm{~s}^{-1}$ (Hillas 1972). The flux of runaway electrons at the end of the avalanche region is then

$$
F_{R R E A}=F_{o} \exp (\xi), \quad \text { where } \xi=\int_{0}^{L} \frac{d z}{\lambda},
$$

where $\lambda$ is the avalanche length shown in Fig. 2. In Eq. (2.2), $\xi$ is the number of e-folding lengths and is equal to $L / \lambda$ for a uniform field.

Fig. 2 Avalanche length calculations (data points) and empirical fits (solid lines) versus electric field strength at sea-level by several authors. The vertical dashed line is the runaway electron avalanche threshold field (Dwyer 2003). As can be seen, there is good agreement between the different simulations, giving confidence in the calculated values of the avalanche length

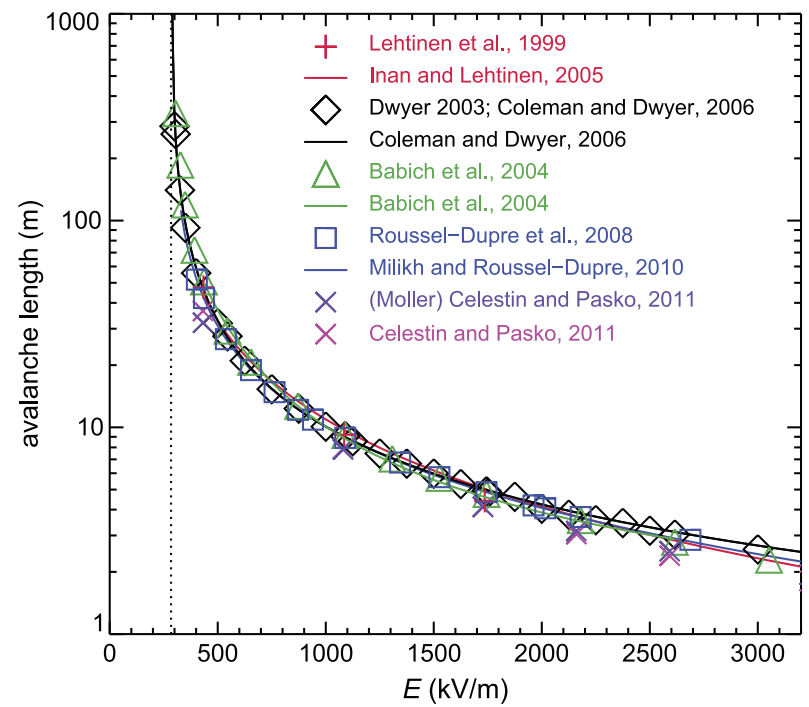


The avalanche (e-folding) length may be written approximately as

$$
\lambda \approx \frac{7.3 \mathrm{MeV}}{e E-F_{d}}
$$

where $F_{d}=0.276 \mathrm{MeV} / \mathrm{m} \times n$ is approximately equal to the average energy loss rate experienced by the minimum ionizing electrons along the avalanche direction (Coleman and Dwyer 2006; Dwyer 2003). We present this expression, which appears as the solid black curve Fig. 2, because it helps illustrate the connection between the avalanche length and the energy spectrum. Other more complicated empirical expressions may be found in the literature cited in Fig. 2.

\subsubsection{Energy Spectra Comparison}

After a few avalanche lengths, the energy spectrum reaches a steady state. For instance, the number of runaway electrons per unit energy may be written approximately as $f_{r e}(\varepsilon) \exp (t / \tau)$, where $\tau$ is the avalanche e-folding time. To find the energy spectrum, $f_{r e}(\varepsilon)$, consider the average kinetic energy gained by a runaway electron that moves a distance $z$ in a constant field: $\varepsilon=z\left(e E-F_{d}\right)$, where $\left(e E-F_{d}\right)$ is the net force experienced by the minimum ionizing electrons along the avalanche direction. Equation (2.2) gives the total number of runaway electrons as a function of distance from the start of the avalanche region. We can equivalently write the number of runaway electrons that traveled a distance between $z$ and $z+d z$, since being created, as $F_{o} \exp ((L-z) / \lambda) d z / \lambda$. Using $\varepsilon=z\left(e E-F_{d}\right)$ to change the variable from $z$ to $\varepsilon$ and Eq. (2.3) then give the energy spectrum of the runaway electrons (runaway electrons per unit energy)

$$
f_{r e}=\frac{F_{R R E A}}{7.3 \mathrm{MeV}} \exp \left(\frac{-\varepsilon}{7.3 \mathrm{MeV}}\right),
$$

valid for runaway electrons above a few hundred $\mathrm{keV}$ up to several tens of $\mathrm{MeV}$.

Equation (2.4) is also correct for electric fields that vary with position. Note that Eq. (2.4), which is independent of the electric field and the density of air, gives an average energy for the runaway electrons of $7.3 \mathrm{MeV}$. Figure 3 shows the average energy of runaway electrons in a RREA as a function of electric field at sea level as calculated from Monte Carlo simulations and from the Boltzmann Equation. Given the differences in how the average energies were calculated, the results are generally in good agreement, showing that the average energy of the runaway electrons in a RREA is about $7 \mathrm{MeV}$ over a wide range of electric field strengths. Note that this currently accepted average energy of runaway elections in a RREA differs substantially from earlier work (e.g. Roussel-Dupré et al. 1994; Symbalisty et al. 1998). In the figure, results from the REAM Monte Carlo simulation used in Dwyer (2003) and Coleman and Dwyer (2006) are shown (labeled Dwyer et al.), along with results from Roussel-Dupré et al. (2008) and Babich et al. (2004a).

The energy spectrum in Eq. (2.4) is approximately valid up to the maximum kinetic energy of the runaway electrons, determined by the potential difference in the high field region and the electric field strength, and falls off quickly above that energy. As can be seen in Fig. 1, the rate that the electrons (and positrons) lose energy slowly increases with kinetic energy due to the relativistic rise and bremsstrahlung energy losses. For electric field just barely above the runaway threshold, $E_{t h}$, the maximum energy will be low and Eq. (2.4) is no longer a good approximation. This can be seen as a reduction in the average energy in Fig. 3. As the electric field strength is increased, the maximum energy also increases. In principle, runaway electrons could reach many hundreds of $\mathrm{MeV}$ in energy as long as there 
Fig. 3 Average energy of runaway electrons in a RREA as a function of electric field at sea level. Note that the Babich et al. results include all electrons above $1 \mathrm{keV}$ (Babich, private communications), which includes both the runaway electron and intermediate energy electron populations, and so the average is slightly lower than that of just the runaway electrons. The vertical dotted line is the runaway electron avalanche threshold field (Dwyer 2003). The solid horizontal line is the $7.3 \mathrm{MeV}$ average energy expected using a simple analytical model

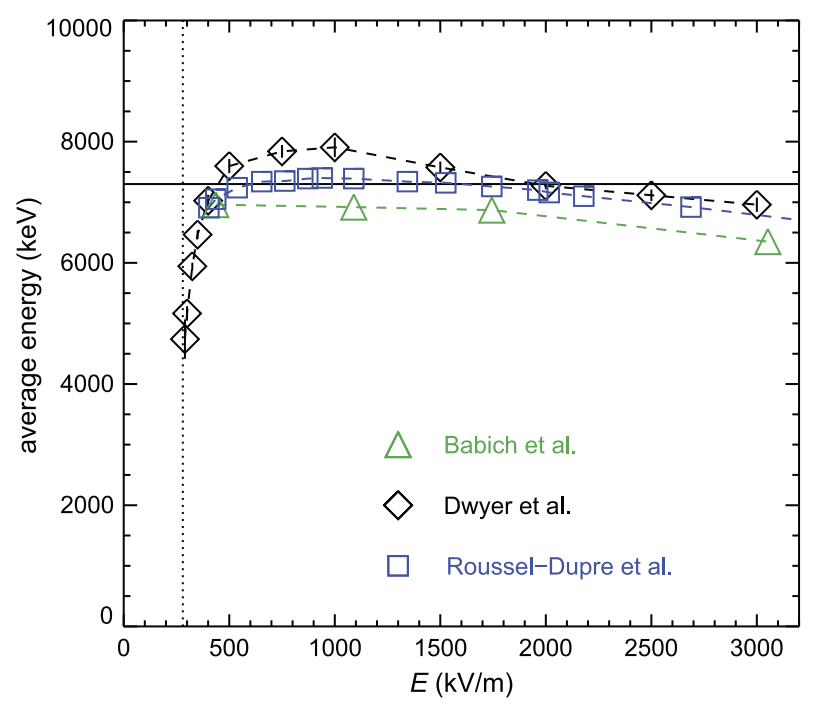

Fig. 4 Electron energy spectrum produced by the RREAs. Both the number density per unit energy found at fixed time and the differential flux of runaway electrons passing through a fixed location are shown. The data points are all from Monte Carlo simulations. The solid curve is a simple analytical model. Above a few hundred $\mathrm{keV}$, the solid curve is the exponential $e^{-\varepsilon / 7.3 \mathrm{MeV}}$ spectrum for the runaway electrons (Dwyer and Babich 2011)

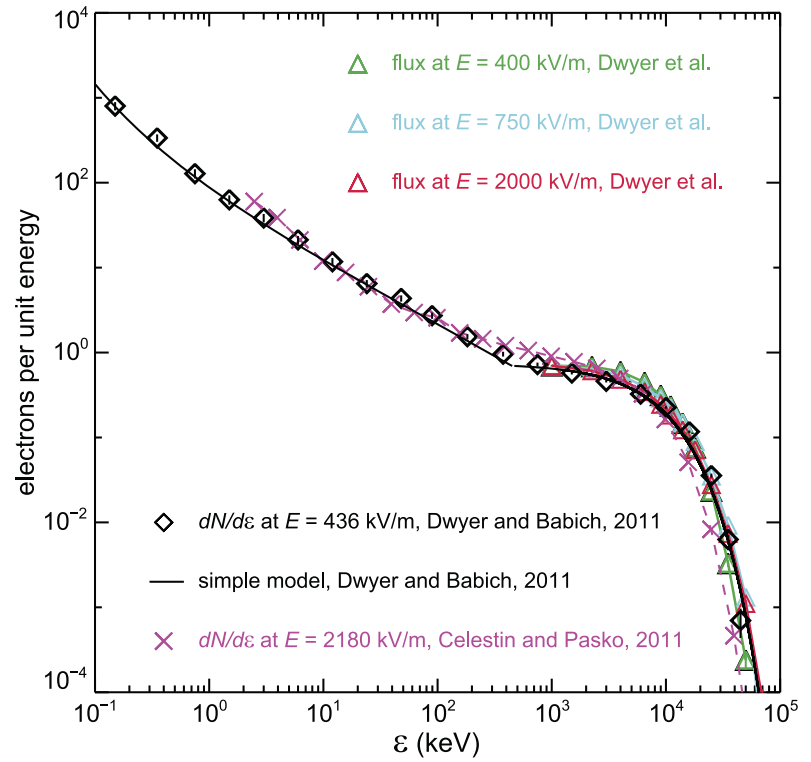

is a large enough electric potential difference in the high field region. As will be discussed further below, relativistic feedback may also limit the maximum possible energy.

Figure 4 shows the energy spectrum of the electrons produced by a RREA as a function of the sea-level equivalent electric field. In the figure, the electron density per unit energy, $d N / d \varepsilon$, is shown as calculated using Monte Carlo simulations by Dwyer and Babich (2011) and Celestin and Pasko (2011) along with a simple analytical model presented in Dwyer and Babich (2011). In particular, above a few hundred $\mathrm{keV}$, the analytical model is the same as Eq. (2.4), which shows good agreement with the more detailed Monte Carlo calculations. According to Celestin and Pasko (2010), these results are also in good agreement with earlier work by Lehtinen et al. (1999). Because the bremsstrahlung x-ray emissions depend 
Fig. 5 Lateral (perpendicular to avalanche direction) and longitudinal (along avalanche direction) diffusion coefficients of the runaway electrons in a RREA determined by Monte Carlo simulations. The solid lines are from Dwyer (2010). The dashed lines are from Babich and Bochkov (2011)

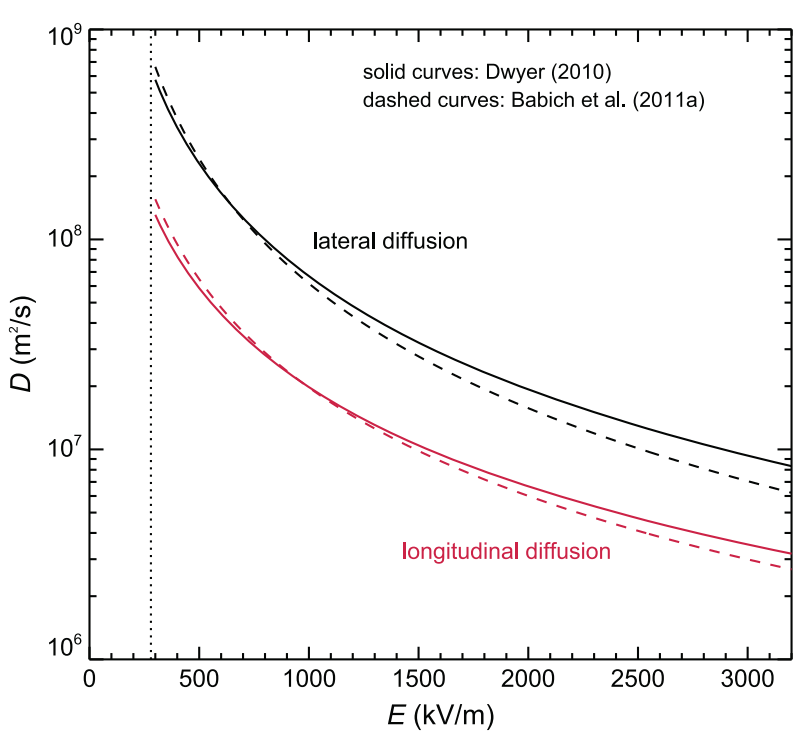

upon the flux of runaway electrons passing through a given location, rather than the number density, the flux of runaway electrons at the end of the avalanche region is shown for several electric field strengths as found by the REAM Monte Carlo simulations (e.g. Coleman and Dwyer 2006; Dwyer 2003).

\subsubsection{Diffusion Coefficients}

Another important quantity for describing RREAs is the lateral and longitudinal diffusion of the runaway electrons in the avalanche. Gurevich et al. (1994) calculated the lateral diffusion of the runaway electrons in an avalanche. Gurevich et al. (1996) investigated the effects of the Earth's geomagnetic field and calculated lateral diffusion coefficients, finding their answer to be in good agreement with the Gurevich et al. (1994) results. More recently, Dwyer (2010) used detailed Monte Carlo simulations to calculate the diffusion coefficients of the runaway electrons and found the lateral diffusion coefficients to be between 15 and 40 times larger than the earlier results. The difference can be attributed to the fact that Dwyer (2010) included elastic scattering of the propagating runaway electrons as well as the initial momentum of the scattered electrons. The earlier work only included the latter, which only makes a minor contribution to the diffusion. Babich and Bochkov (2011) used Monte Carlo simulations to calculate the diffusion coefficients and found values similar to the work by Dwyer (2010) (see Fig. 5). Because the lateral diffusion is so much larger than earlier calculations, Dwyer (2010) questioned the peak conductivity calculations in earlier work, which were used in lightning initiation models.

\subsubsection{Concluding Remarks}

In summary, recent work by several groups appears to be in good agreement for key parameters that describe relativistic runaway electron avalanches (RREAs) in our atmosphere, including the avalanche threshold field, the avalanche length (and time), the propagation speed of the avalanche, and the lateral and longitudinal diffusion coefficients. A remaining point of contention is the impact that RREAs have on the conductivity of the air. In particular, a 
great deal of attention has gone into the implications of large runaway election avalanches seeded by cosmic-rays. Gurevich et al. (1992) suggested that runaway electron avalanches will lead to an electrical breakdown of air. This hypothesized electrical breakdown generated by a RREAs has been named "runaway breakdown" (Gurevich and Zybin 2001). Gurevich et al. (1999) proposed that a cosmic-ray extensive air shower seeding a RREA could produce a large enough conductivity enhancement to initiate lightning. Furthermore, several authors have claimed that RREAs, initiated by cosmic-rays, result in anomalously large conductivity increases, much larger than would be calculated using the flux of energetic runaway electrons and standard ionization calculations (Gurevich and Milikh 1999; Gurevich et al. 1999, 2004a). Dwyer (2005b, 2010) and Dwyer and Babich (2011) have challenged these conductivity calculations, demonstrating that standard ionization rates apply to RREAs. They further argued against the use of the term "runaway breakdown," since RREA is not really an electrical breakdown as the term is usually used (also see Dwyer 2007). Because the flux of runaway electrons produced by RREAs is dependent upon conditions external to the system (see Eq. (2.2)), RREAs cannot be considered an electrical breakdown, which is an internal state of the system. This situation is analogous to low-energy electron avalanches, such as occur in gas filled proportional counters, which are not considered an electrical breakdown. Indeed, Babich et al. (2002) observed the initial development of relativistic runaway electron avalanches in the laboratory without accompanying electrical breakdown (also see Babich et al. 2004b), illustrating that RREAs are not synonymous with breakdown. As a result, in this review, we shall refer to the avalanche multiplication of relativistic runaway electrons, as first described by Gurevich et al. (1992) as RREAs. We shall not use the name "runaway breakdown," since we consider it to be a misnomer, and there is currently no evidence that a RREA can actually produce any form of electrical breakdown.

\subsection{Thermal Runaway Electrons}

In this section we discuss the thermal runaway electron process, especially as a potential source of seed particles for Wilson runaway and RREA. As can be seen in Eq. (2.2), the flux of runaway electrons from the RREA mechanism is proportional to the flux of energetic seed electrons. In addition to external sources of seed electrons, it is possible for lightning leaders and/or streamers to supply the energetic seed electrons internally. If in some small region the electric field exceeds the critical field, as seen in Fig. 1, then runaway electrons may be accelerated out of the low-energy population, via the thermal runaway mechanism (Gurevich 1961; Sizykh 1993).

High enough fields to produce thermal runaway may exist at streamer heads or leader tips. The thermal runaway electron process alone cannot account for the energetic radiation recorded in our atmosphere, since the average energy from this process is too low (e.g. a few keV) (Moss et al. 2006). However, these runaway electrons may continue to gain energy from the field, even if the field grows weaker with distance. We envision two basic scenarios: In the first scenario, the thermal runaway process provides the seeds for Wilson runaway. In this case, one energetic electron is produced for every seed runaway electron created by the thermal runaway process. The Wilson runaway mechanism increases the energy of the runaway electrons and extends the distance traveled, thereby increasing the $\mathrm{x}$-ray yield. Because the total energy gained will depend upon the electric field configuration, there is no characteristic energy for these runaway electrons. In the second scenario, if the high field region is increased so that the runaway electrons traverse a greater potential difference, either by increasing the field strength or the spatial extent of the high field $\left(E>E_{t h}\right)$ region, 
then the Wilson runaway electron mechanism turns into RREA and additional MeV runaway electrons are generated through avalanche multiplication. In this case, many runaway electrons can be generated for each seed electron injected by the thermal runaway process and the average energy of the runaway electrons becomes $\sim 7 \mathrm{MeV}$.

Because the critical field, $E_{c}$, is about 10 times larger than the conventional breakdown field $\left(E_{k}=3 \times 10^{6} \mathrm{~V} / \mathrm{m} \times n\right)$ (Moss et al. 2006), until relatively recently in 2004, it was not clear whether or not conditions exist in the atmosphere that allow thermal runaway to occur, since such high fields should discharge on very short timescales (Babich 2003; Bakhov et al. 2000). When it was established that lightning emits x-rays (Moore et al. 2001; Dwyer et al. 2003), many researchers assumed that this energetic emission was produced by the RREA mechanism acting on cosmic-rays. However, based upon the energy spectra and flux of xrays from rocket-triggered lightning, Dwyer (2004) showed that the RREA mechanism was not consistent with the observations and suggested that the thermal runaway mechanism was responsible for the energetic radiation. The next year, the discovery of x-ray emission from long laboratory sparks in air, similar to the emission seen from lightning, supported this hypothesis (Dwyer et al. 2005a). Several groups have modeled thermal runaway electron production from streamers, with applications to lightning and laboratory sparks (Moss et al. 2006; Li et al. 2009; Chanrion and Neubert 2008, 2010; Colman et al. 2010; Celestin and Pasko 2011).

Gurevich et al. (2007) also modeled runaway electron production by lightning stepped leaders. In their paper, they described a two-step mechanism in which runaway electron avalanche multiplication in the high-field regime acts upon thermal runaway electrons generated by the lightning leader/streamers. The idea is similar to thermal runaway augmented by RREA as discussed above. According to their paper, the difference is that the avalanche multiplication occurs in very high fields where the avalanche lengths are short. In their paper, they calculate that the avalanche length decreases as $1 / \delta^{2}$ in the high-field regime and $1 / \delta^{3 / 2}$ in the low-field, RREA regime, where $\delta=E / E_{b}$. They then estimate that the avalanche length near the critical field $E_{c}(\delta=100)$ is about $1 \mathrm{~cm}$. The problem with this argument is that $1 / \delta^{3 / 2}$ disagrees with the accepted $\sim 1 / \delta$ dependence of the avalanche length (see Sect. 2.2.3), which is found to be valid up to at least $\delta=25$ (Milikh and Roussel-Dupré 2010; Coleman and Dwyer 2006). Even if we, for the moment, stipulate that the field dependency somehow steepens dramatically above $\delta=25$, taking the accepted avalanche length at $\delta=25$ and extrapolate it to $\delta=100$ gives an avalanche length of $7 \mathrm{~cm}$, not the $1 \mathrm{~cm}$ as claimed in the Gurevich et al. paper. On the other hand, using the standard $1 / \delta$ dependence gives an avalanche length of $30 \mathrm{~cm}$ at $\delta=100$. Either way, these longer avalanche lengths violate the assumption used in the Gurevich et al. (2007) paper that the avalanche length is small compared with the size of the streamer head. In addition, the $1 \mathrm{~cm}$ avalanche length for $\delta=100$ estimated by Gurevich et al. (2007) implies an average runaway electron energy of no more than $218 \mathrm{keV}$, which disagrees with Monte Carlo calculation using ELIZA by Babich and Bochkov (2011), who found an average energy of $3.46 \mathrm{MeV}$ at $\delta=100$.

\subsection{Bremsstrahlung and Photon Interactions}

Because x-rays and gamma-rays propagate much farther through the atmosphere than energetic electrons, it is important to consider the energetic radiation produced by the runaway electrons as they interact with air. Although there are several mechanisms for generating energetic radiation, i.e., x-rays and gamma-rays, in our atmosphere, the single most important mechanism is bremsstrahlung interactions of energetic electrons (and positrons) with air atoms. Technically, any energetic photon $(>1 \mathrm{keV})$ that is generated by an electron is 
called an x-ray and energetic photons that are generated by other processes, such as nuclear decays, are called gamma-rays. As a result, nearly all the energetic photons discussed in this review are x-rays. The main exception is the $511 \mathrm{keV}$ positron annihilation gamma-rays. Alternatively, photons with energies greater than about $1 \mathrm{MeV}$, regardless of how they were produced, are sometimes called gamma-rays. In this review, we shall adopt the convention of calling energetic photons either x-rays or gamma-rays, depending upon the source, the instrument that observes them, and the energy of the photon. For example, since TGFs were first observed by an astrophysics gamma-ray observatory (CGRO), these photons are always referred to as gamma-rays, even though their energies (which extend down to tens of keV) may overlap with the $\mathrm{x}$-ray range and they were created by bremsstrahlung. Because the TGFs appear to originate from thunderclouds and thunderclouds also emit longer duration energetic radiation with the same spectrum, we adopt the term gamma-ray glows for these longer emissions. On the other hand, the energetic photons observed from lightning and laboratory sparks are appropriately called x-rays.

The differential energy spectrum of bremsstrahlung emission from an electron with kinetic energy $\varepsilon$, is approximately a power law with index -1 , i.e. $1 / \varepsilon_{p}$, up to the energy $\varepsilon$. Since the RREA spectrum is approximately $e^{-\varepsilon / 7.3 \mathrm{MeV}}$, and the bremsstrahlung spectrum emitted by the runaway electrons in a RREA is the convolution of $1 / \varepsilon_{p}$ with this electron source spectrum, the approximate photon spectrum is then $\varepsilon_{p}^{-1} e^{-\varepsilon_{p} / 7.3 \mathrm{MeV}}$, which is reasonably close to the exact spectrum. Furthermore, the bremsstrahlung photons are emitted in a forward beam with a half angle equal to about one over the Lorentz factor of the energetic electrons (Koch and Motz 1950).

Once x-rays or gamma-rays are emitted, they interact with air primarily in four ways: Compton scattering, photo-electric absorption, pair production and Rayleigh scattering (Berestetskii et al. 1982). From about $100 \mathrm{keV}$ up to several MeV, Compton scattering almost completely dominates these interactions. Compton scattering not only changes the direction of the photon, it causes the energy to be reduced. As photons experience multiple Compton scatters, they tend to accumulate around $100 \mathrm{keV}$ in the Compton peak. Below this energy the photons are lost to photoelectric absorption. Above a few $\mathrm{MeV}$, pair production becomes important and electron-positron pairs are generated. These positrons may play an important role in high-energy atmospheric processes, as will be discussed below. Rayleigh scattering, which simply changes the direction of the photon propagation without changing the energy of the photon, usually plays a minor role in the photon propagation at these energies.

Because the hardest (flattest) that the bremsstrahlung spectrum can be is $1 / \varepsilon_{p}$ at the source, and in reality the runaway electron spectrum makes the gamma-ray spectrum even steeper, observing a spectrum that is flatter than $1 / \varepsilon_{p}$, especially near $1 \mathrm{MeV}$, indicates that the gamma-rays have traveled through a large amount (e.g. many ten of $\mathrm{g} / \mathrm{cm}^{2}$ ) of air. This method was used with the RHESSI TGF data to infer the source altitude of TGFs, as will be discussed below (Dwyer and Smith 2005).

\subsection{Radio Emissions from Cosmic-Ray Extensive Air Showers and RREA}

In addition to energetic radiation, runaway electrons may also produce radio emissions that may be measured remotely. The ambient steady state cosmic-ray background may serve as seed particles. Cosmic-rays also arrive in the form of extensive air showers (EASs), composed sometimes of millions of energetic particles propagating down through the atmosphere at once. At thundercloud altitudes most of these charged particles are electrons and positrons. These air showers can impulsively seed RREAs, resulting in a large avalanche 
of runaway electrons that may last just a few microseconds. The possibility that such air showers seeding RREAs may initiate lightning will be discussed further below (Sect. 5).

Roussel-Dupré and Gurevich (1996) presented an equation, without derivation, for the radio emissions from a single RREA. Later, Dwyer et al. (2009) presented a derivation of the radio emission from a RREA and found a somewhat different equation than that presented by Roussel-Dupré and Gurevich (1996). Starting with Gurevich et al. (2002), several papers have calculated the radio-frequency (RF) emission from cosmic-ray extensive air showers and relativistic runaway electron avalanches (Gurevich and Zybin 2004; Gurevich et al. 2004b, 2004c, 2006). Tierney et al. (2005) attempted to model narrow bipolar events (NBEs) - powerful radio pulses from thunderclouds-by developing a one-dimensional RREA model to investigate RF emissions produced inside thunderclouds, but inexplicably applied it to individual cosmic-ray seed electrons rather than extensive air showers. Because a region of a thundercloud just $100 \mathrm{~m}$ across would inject about $10^{8}$ such seed particles per second, it is difficult to see how this scenario would account for NBEs, which are isolated events.

There exists considerable literature regarding radio emission from cosmic-ray air showers not involving runaway electron avalanche multiplication (e.g., Jelley et al. 1965; Tompkins 1974; Buitink et al. 2007; Ender et al. 2009). Most of this work involves synchrotron emission of electrons and positrons in the shower moving in the geomagnetic field (Kahn and Lerche 1966). However, this geosynchrotron emission from the air showers is usually quite small (e.g., usually measured in $\mu \mathrm{V} \mathrm{m}^{-1} \mathrm{MHz}^{-1}$ ) and is highly beamed in the direction of the shower, with the electric field strength decreasing exponentially with an efolding distance of about $100 \mathrm{~m}$ from the shower center (Huege and Falcke 2005). Buitink et al. (2010a, 2010b) performed CORSIKA simulations and found that the number of electrons in an air shower increases dramatically when the electric field exceeds the runaway electron threshold field, enhancing the geosynchrotron RF emissions. However, they did not include the RF emissions from the growing or decaying currents caused by the RREA production. There is currently great interest in the ultra-high energy cosmic-ray physics community in better understanding how the RF emissions, via geosynchrotron emission and/or RREA, are modified by atmospheric electric fields.

For the RREA mechanism, because the seed particles from an air shower will be injected over a very short time period, the runaway electron number will grow approximately exponentially with time as the avalanche develops, and then decay approximately exponentially with time as the runaway electrons propagate out of the avalanche region (Dwyer et al. 2009). As they propagate, the runaway electrons will ionize the air, generating a large number of secondary low-energy (few eV) electrons. These low-energy electrons will drift in the electric field producing an electrical current that is much larger than the current from the runaway electrons. Because the low-energy electrons quickly attach to air, the current source from the low-energy electrons closely follows the avalanche front of the runaway elections, moving with the same speed as the avalanche (i.e., $0.89 \mathrm{c}$ ) even though the individual low-energy electrons are moving at much lower speeds. Dwyer et al. (2009) developed a detailed model that includes inclined trajectories of realistic air showers, currently accepted avalanche lengths and standard ionization rates. Figure 6 shows results of the numerical simulations from Dwyer et al. (2009).

When the effects of RREA are included, the amplitude of the RF pulses are increased by orders of magnitude over normal geosynchrotron emissions. In additional, the RF pulses may be measured over distances of many $\mathrm{km}$ from the air shower core, rather than hundreds of meters. Finally, Dwyer et al. (2009) pointed out that it may be possible to use the pulse shape of the RF emission to remotely measure the quasi-static electric fields inside the thundercloud, measurements that are very difficult to make with standard techniques. 


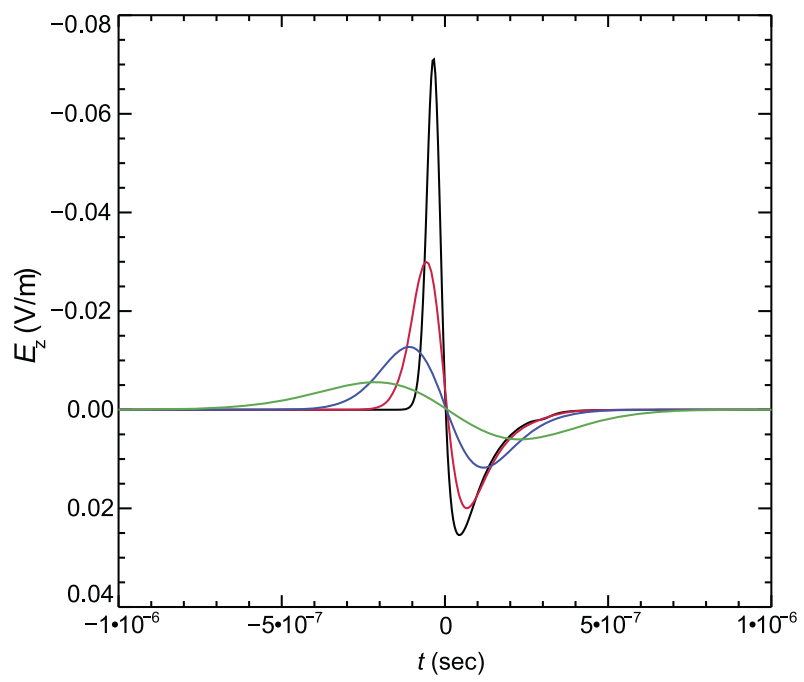

Fig. 6 Simulations of the pulse shape of the vertical electric field versus time as measured near the ground by an antenna located near an air shower core. The black curve is for a thundercloud electric field of $1250 \mathrm{kV} / \mathrm{m}$; the red curve is for $625 \mathrm{kV} / \mathrm{m}$; the blue curve is $375 \mathrm{kV} / \mathrm{m}$, and the green curve is $250 \mathrm{kV} / \mathrm{m}$. The high field regions are all located at an altitude of $5 \mathrm{~km}$ and the fields are directed upward. For each thundercloud field strength, the depth of the avalanche region was chosen to correspond to 10 avalanche lengths. The simulations used a $10^{17} \mathrm{eV}$ air shower at $45^{\circ}$ with respect to vertical. As can be seen, the pulse shape measured remotely on the ground is extremely sensitive to the electric field strength inside the thundercloud. From Dwyer et al. (2009)

\subsection{Relativistic Feedback Mechanism}

\subsubsection{Overview}

Dwyer (2003) introduced a new runaway electron production mechanism that involves positive feedback effects from positrons and energetic photons, the inclusion of which results in a dramatic change of behavior that cannot be explained by the RREA mechanism alone. In this mechanism, illustrated in Fig. 7, avalanches of runaway electrons emit bremsstrahlung Xrays that may either Compton backscatter or pair-produce in air. If the backscattered photons propagate to the start of the avalanche region and produce other runaway electrons, either via Compton scattering or photoelectric absorption, then a secondary avalanche is created (also see Babich et al. 2007c). Alternatively, the positrons created by pair-production often turn around in the ambient electric field and run away in the opposite direction of the electrons. Because the positron annihilation cross-section decreases with energy and the positrons quickly accelerate to many ten of $\mathrm{MeV}$, the positrons usually travel on the order of a kilometer at sea level before annihilating. If these positrons propagate to the start of the avalanche region they can produce additional runaway electrons via hard elastic scattering with atomic electrons in the air (i.e. Bhahba scattering), thereby producing secondary avalanches. These secondary avalanches may in turn emit more x-rays that Compton scatter or pair-produce, resulting in more feedback and more avalanches. As a result, the number of runaway electron avalanches increases exponentially on a timescale measured in microseconds (Dwyer 2003; Babich et al. 2005). These feedback mechanisms are analogous to the feedback mechanisms that occur at low energies during a Townsend discharge. For example, in a Geiger counter, 


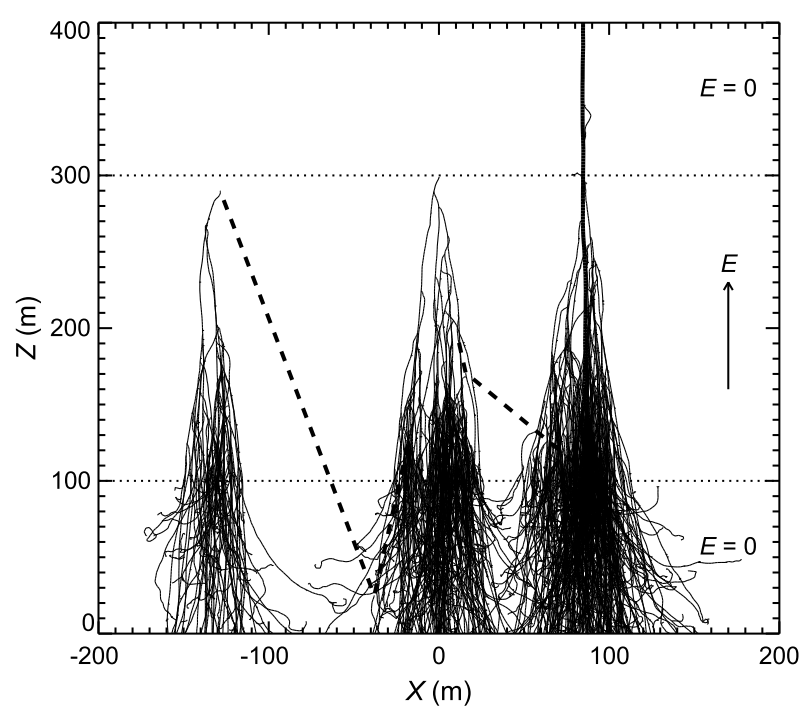

Fig. 7 The relativistic feedback mechanism. Partial results of the Monte Carlo simulation are shown. The light tracks are the runaway electrons, the dashed lines are the x-rays and the dark track is a positron. The entire avalanche is initiated by one, $1 \mathrm{MeV}$, seed electron injected at the top of the high field region $(x=0$, $z=300 \mathrm{~m})$. The horizontal dotted lines show the boundaries of the electric field volume $(E=1000 \mathrm{kV} / \mathrm{m})$. For clarity, only a small fraction of the runaway electrons and x-rays produced by the avalanche are plotted. The avalanches on the left and right illustrate the x-ray feedback and positron feedback mechanisms, respectively. Figure from Dwyer (2003)

UV photon and ion collisions at the cathode generate a self-sustained breakdown, which terminates only when the voltage collapses. To distinguish the feedback mechanisms described here, which involve high-energy particles, from the low-energy feedback mechanisms occurring in ordinary Townsend gas discharges, these high-energy feedback mechanisms are jointly referred to as relativistic feedback (Dwyer 2007).

\subsubsection{Properties of the Relativistic Feedback Mechanism}

Dwyer (2003) showed that large electric fields are highly unstable due to relativistic feedback. Relativistic feedback may naturally explain very large fluxes of energetic electrons and gamma-rays (e.g., TGFs), and it also severely limits the electric field regimes in which alternative mechanisms (i.e., runaway breakdown) may operate. In particular, relativistic feedback limits the amount of runaway electron avalanche multiplication that is possible, as shown in Fig. 8. This result raises questions about other work that relies on large avalanche multiplication factors (see Dwyer and Rassoul 2011).

An important parameter for describing relativistic feedback is the feedback factor, $\gamma$, which is the fractional increase or decrease in the number of runaway electrons during each feedback cycle of duration $\tau_{f b}$. The feedback time, $\tau_{f b}$, is the time for the runaway electrons and backward propagating positrons or x-rays to complete one round trip within the avalanche region.

If $\gamma<1$, in the steady state, the feedback process enhances the flux of runaway electrons in Eq. (2.2) by a simple multiplicative factor:

$$
F_{R R E A}=\frac{F_{o} \exp (\xi)}{1-\gamma},
$$


Fig. 8 Maximum sustainable relativistic runaway electron avalanche multiplication factor, $\exp \left(\xi_{0}\right)$, versus total potential difference within the avalanche region. The data points (and the dashed and dashed-dotted lines) are the result of Monte Carlo simulations and show the threshold at which the discharge becomes self-sustaining $(\gamma=1)$. The data are calculated for the condition that the lateral radius, $R$, is much larger than the length of the avalanche region, $L$, and when it is one half the length. Figure from Dwyer (2008)

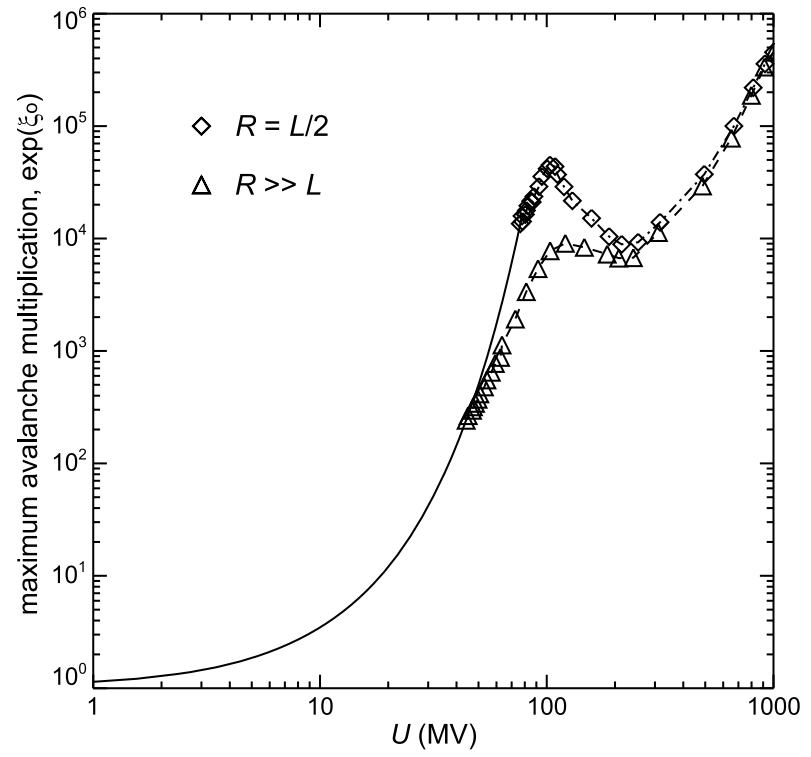

where $\xi$ is the number of e-folding lengths. In Eq. (2.5), $F_{o} /(1-\gamma)$ is the flux of seed runaway electrons from both external sources, e.g. cosmic-rays, and from relativistic feedback. If the avalanche multiplication is increased, for example from a field increase due to thundercloud charging, causing the feedback factor to approach $\gamma=1$, then the flux of seed runaway electrons in Eq. (2.5) will become very large, and relativistic feedback will dominate over external seed particles. Indeed, as thunderclouds charge, these runaway electrons may produce a significant discharge current which may under some circumstances balance the charging currents, temporarily establishing an approximate steady-state electric field configuration. This scenario may explain the long lasting gamma-ray glows discussed below.

For a sufficiently rapid growth of the electric field, it is possible to drive $\gamma$ above 1 before the system can respond with a large discharge current. This may be accomplished through the large scale charging of the thundercloud or by charge motion during lightning. In addition, the currents from the RREA mechanism may drive $\gamma$ above 1 for some parts of the avalanche region while discharging other parts (Dwyer 2005b). When $\gamma>1$ and relativistic feedback dominates the production of seed particles, the runaway electron flux at time $t$ is given by

$$
F_{R F} \propto \exp (\xi) \gamma^{t / \tau_{f b}}=\exp (\xi) \exp \left(t / \tau^{\prime}\right)
$$

where

$$
\tau^{\prime}=\tau_{f b} / \ln (\gamma) .
$$

From Eq. (2.6) it can be seen that the feedback cycle increases the number of runaway electrons by a factor of $\gamma$. In a very short time, the flux of seed runaway electrons comes almost entirely from the feedback process. In this case, the discharge becomes self-sustaining, representing a true breakdown, and the external source of seed particles could be completely removed without affecting the runaway electron flux. The rapidly increasing number of runaway electron avalanches generated by the feedback process results in a very large flux of runaway electrons and accompanying gamma-rays. Indeed, Dwyer (2008) showed that the 
relativistic feedback mechanism could naturally account for the large fluences and short times scales of TGFs.

\subsubsection{Comparison with Earlier Mechanisms}

The difference between Wilson's runaway electron mechanism, the RREA mechanism, and the relativistic feedback mechanism are illustrated in Fig. 9. Each mechanism arises from the addition of new processes that were not included in the previous mechanism, the inclusion of which results in dramatically different behavior from the previous mechanism. In a sense, the overarching mechanism is relativistic feedback, since it encompasses the other two. When the avalanche multiplication factor is small (i.e., the feedback factor is small), the relativistic feedback mechanism becomes equivalent to the RREA mechanism. If the avalanche multiplication factor is reduced further, it becomes equivalent to the Wilson runaway electron mechanism.

Milikh and Roussel-Dupré (2010) argued that relativistic feedback can be neglected in many cases, but Dwyer and Rassoul (2011) showed that their argument was incorrect and was apparently based upon confusion about the principle of positive feedback. In particular, Milikh and Roussel-Dupré (2010) incorrectly compared their estimates of the positron production rate with the total runaway electron production rate rather than the seed particle

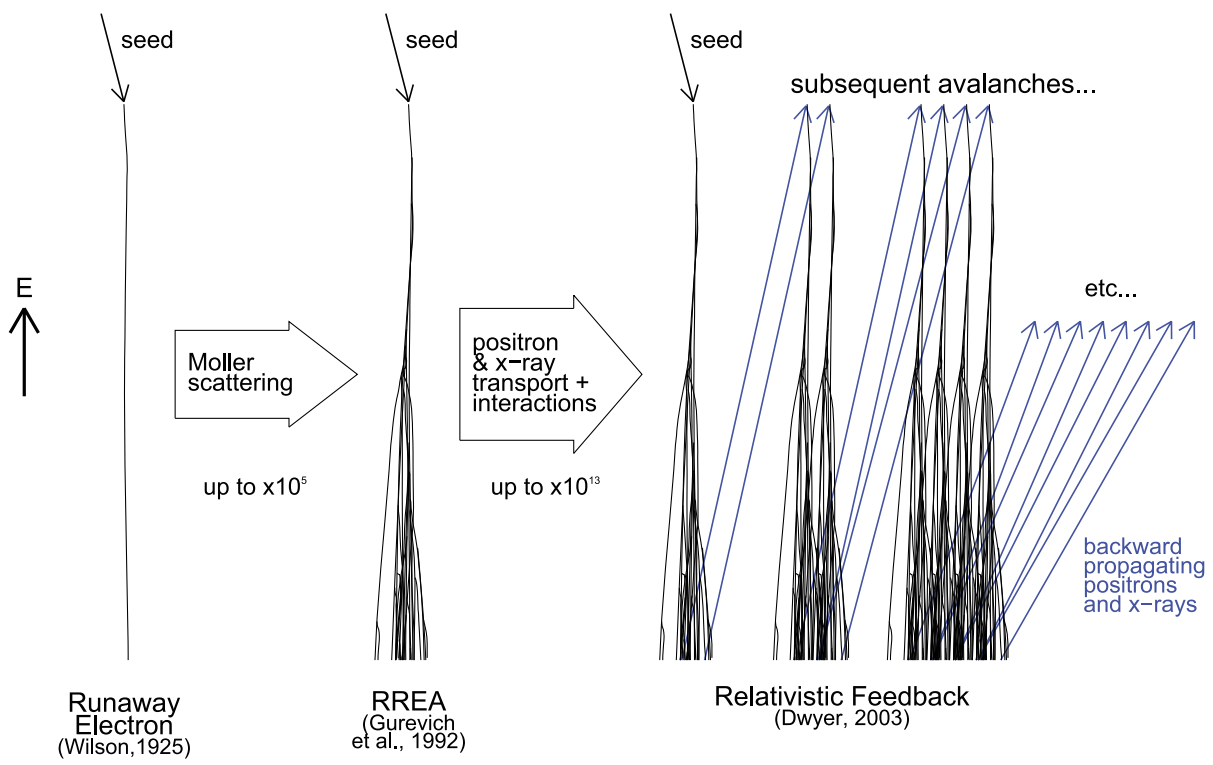

Fig. 9 Schematic diagram summarizing the three mechanisms for generating energetic electrons in an atmosphere. By including Møller scattering in the runaway electron mechanism, a relativistic runaway electron avalanche (RREA) is produced, with an increase in the number of runaway electrons of up to $10^{5}$ over the Wilson runaway electron mechanism. By including positron and x-ray transport and interactions to the RREA mechanism, Relativistic Feedback is produced, with an increase in the number of runaway electrons of up to $10^{13}$ over the RREA mechanism. For clarity, the backward propagating positrons and x-rays (blue arrows) and the resulting subsequent avalanches are offset to the right. In reality, they often overlap the initial avalanche. The energetic seed particle that becomes the first runaway electron may be supplied by atmospheric cosmic-rays, radioactive decays or by thermal runaway electron production during lightning or other sparks 
production rate, which is the relevant comparison for feedback processes. This misunderstanding was reiterated in Milikh and Roussel-Dupré (2011).

In contrast, Dwyer $(2003,2007,2008)$ and Babich et al. (2005) have shown that relativistic feedback is often applicable inside thunderclouds, limiting the electric field and runaway electron avalanche multiplication that may be achieved. Specifically, the positron feedback mechanism usually dominates for average electric fields below about $750 \mathrm{kV} / \mathrm{m} \times n$ and $\mathrm{x}-$ ray feedback usually dominates above that field (Dwyer 2007). The threshold field at which relativistic feedback becomes self-sustaining, i.e., $\gamma=1$, depends upon the details of the electric field and has been calculated for several configurations by Monte Carlo simulations (see Fig. 8) in the references above. One important parameter is the lateral width of the high field region, which affects the x-ray feedback rates in particular. This effect was studied in Dwyer (2007, 2008).

\section{Observations of Processes in High Energy Atmospheric Physics}

\subsection{Early Observations of High-Energy Radiation from Thunderstorms}

Wilson's runaway electron proposal led to a number of experiments to observe energetic electrons or X-rays from thunderstorms (e.g., Schonland 1930; Schonland and Viljoen 1933; Appleton and Bowen 1933; Macky 1934; Halliday 1934, 1941; Clay et al. 1952; Hill 1963; Shaw 1967; Whitmire 1979; D'Angelo 1987; also see Suszcynsky et al. 1996 and Babich 2003 for excellent overviews). Some of these papers reported positive results, and in hindsight, some probably were reporting real x-ray or gamma-ray emissions from thunderclouds and lightning. However, much of the early work was inconclusive, and as of about 1980 there appeared to be no consensus that either thunderclouds or lightning emitted energetic radiation (Suszcynsky et al. 1996).

A problem that applied to some earlier work and still applies to some recent measurements is that care was not taken to insure that the signals, interpreted as from x-rays or other energetic particles, did not arise from spurious EM sources. Thunderstorms and lightning produce electromagnetically noisy environments in a frequency range that may be picked up in power systems and otherwise by the sensitive electronics used to measure the energetic radiation. As a result, measurements based solely upon count rates of signals above some discriminator threshold should be viewed with caution, since it is not obvious what is being counted, pulses from energetic particles or, for instance, RF noise from lightning processes. Gain fluctuations due to voltage changes in the electronics may also be an issue when lightning is in the area. Ideally, when making measurements near thunderstorms and lightning, detectors and electronics should be placed inside metal boxes or Faraday cages and be powered by batteries inside the cages or by local power supplies/generators (not the utility grid). Long lengths of conductive power or signal cables should be avoided. Comparison of outputs from control detectors that have the same electronics chains as the active detectors are one method to guard against false measurements. It is also very helpful to record the waveforms of the detector signals that are being measured rather than just count rates. Detectors designed primarily to count photons, charged particles, or neutrons often have significant response to the other classes of particle as well, and this response must be carefully simulated before final conclusions are drawn about the nature of the signal being observed. Finally, the gamma-ray emissions from radon daughter products are a known source of background, which may vary dramatically with time, especially increasing when precipitation is present. The reader is encouraged to be suspicious of measurements that do not address all of the issues listed above. 


\subsection{Thunderstorm Gamma-Ray Glows}

We use the term glow to refer to any high-energy photon emission from a thundercloud that has a time scale longer than 1 second, the typical maximum duration for a lightning flash. This is in contrast to the shorter duration, pulsed photon emissions discussed in Sects. 3.3 and 3.4.

\subsubsection{Airborne Observations of X-Ray/Gamma-Ray Glows}

The modern era of observations of high-energy radiation in thunderstorms began with the airplane flights of Parks and collaborators from the University of Washington (Parks et al. 1981; McCarthy and Parks 1985). A NASA F-106 jet carrying NaI scintillation detectors with thin entrance windows was flown directly into active thunderstorm cells. In the first paper, x-ray energies were only resolved below $12 \mathrm{keV}$, so it was not yet apparent that $\mathrm{x}$-rays in storms extend to much higher energies. Although there was some evidence of anticorrelation between lightning and the high-energy radiation even in these first data, it was not significant enough to overcome the natural presumption that the high-energy radiation was directly associated with lightning. The later flights in 1984, however, demonstrated beyond question that the $\mathrm{x}$-rays go to higher energies (at least $>110 \mathrm{keV}$ ), that the high-energy radiation has a longer time scale than a lightning flash (sometimes varying smoothly over tens of seconds), and that lightning, either nearby or striking the plane, generally terminates, rather than causes, the x-ray glows (McCarthy and Parks 1985). Figure 10, from McCarthy and Parks (1985), shows both the gradual nature of the glows while they are active and their abrupt termination due to lightning.

In the following decade, Eack and collaborators used balloons to carry both scintillators and electric field detectors through and above active cells (Eack et al. 1996a, 1996b, 2000). A hard x-ray glow (measured up to $120 \mathrm{keV}$ ), similar to those seen by the University of Washington group, was reported from within an active mesoscale convective system (MCS) at $4 \mathrm{~km}$ altitude by Eack et al. (1996a). The x-ray emission coincided with the altitude of highest electric field (see Fig. 11), although the field at the balloon was only about half the break-even field for $1 \mathrm{MeV}$ runaway electrons. The emission lasted as long as it took the balloon to transit the high-field region, but was interrupted by a pair of lightning flashes that briefly reduced and then restored the x-rays. The authors noted that the field could have reached break-even values at the point of production of the x-rays but not at the balloon. A second sounding found a similar high x-ray flux in an anvil at $14 \mathrm{~km}$, suggesting that this phenomenon can occur in different parts of the charge structure of a storm and is not uncommon (Eack et al. 2000).

Fig. 10 Three $x$-ray excesses from a NASA/University of Washington flight in 1983. The first two events are terminated by a nearby lightning flash and a strike to the plane, respectively. The third ends gradually, possibly as the plane exits the region. From McCarthy and Parks (1985)

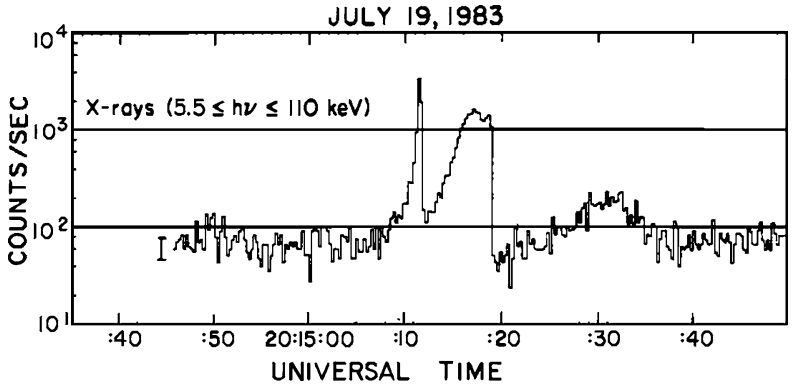


Fig. 11 Electric field (left) and $\mathrm{x}$-ray count rate (right) during a balloon sounding through a MCS. On the left-hand panel, lightning flashes are marked " $L$ " and the predicted break-even field for $1 \mathrm{MeV}$ runaway electrons is marked $E_{B E}$. From Eack et al. (1996a)

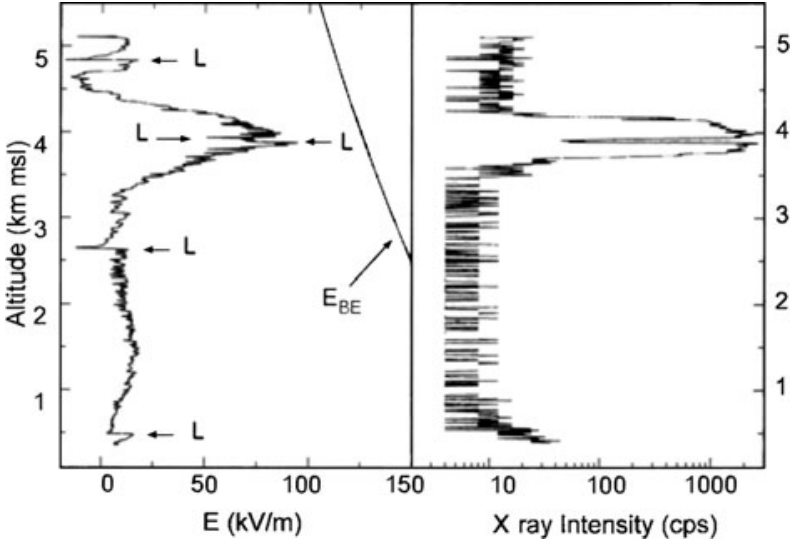

Gurevich and Milikh (1999) modeled the x-ray observations of Eack et al. (1996a) using their runaway breakdown (RREA) model and found good agreement with those observations (also see Milikh and Roussel-Dupré 2010). A problem with both the model and the Eack et al. energy spectra, as presented by Gurevich and Milikh, is that the X-rays are too soft, with the spectra dropping sharply from 60-90 to 90-120 keV, to be consistent with the currently accepted RREA spectrum (see Fig. 21). Specifically, the Gurevich and Milikh spectra decrease 2.5 times more than the RREA spectrum calculated by the REAM Monte Carlo for the same conditions. The Gurevich and Milikh (1999) calculation was based upon the runaway electron spectra of Symbalisty et al. (1998), which produced average runaway electron energies a few times smaller than currently accepted values as shown in Fig. 3 above. However, even taking the incorrect runaway electron energies into account cannot explain the disagreement with the currently accepted gamma-ray spectrum, since the bremsstrahlung source spectrum will always be approximately $1 / \varepsilon_{p}$ as long as the energy range being considered is less than of the energy scale of the electrons. The same problem can be seen in the gammaray spectrum calculated in Gurevich et al. (2001b) [their Fig. 6], which drops precipitously from $60 \mathrm{keV}$ to $200 \mathrm{keV}$. It is possible to obtain a softer energy spectrum by considering the Compton scattered component at large angles, out of the initial gamma-ray beam. However, Gurevich and Milikh (1999) and Gurevich et al. (2001b) only considered cases within the beam. Alternatively, thermal runaway could account for the soft spectrum, which is qualitatively similar to the x-ray emissions from lightning and laboratory sparks (Dwyer 2004; Dwyer et al. 2005a; Moss et al. 2006). Trakhtengerts et al. (2002, 2003) also considered runaway electron production in stochastic (randomly varying) electric fields inside thunderclouds as possible explanation for these x-ray emissions.

Another balloon flight found three shorter x-ray pulses (the authors' term) of about 1 second each, seen when the payload was at $15 \mathrm{~km}$ altitude, $3 \mathrm{~km}$ above an MCS, with no significant electric field at the balloon payload (Eack et al. 1996b). Several lightning flashes were occurring within the MCS at the same time as these pulses, including both positive and negative cloud-to-ground events, but all had strike points over $100 \mathrm{~km}$ away. This is too great a horizontal distance, by at least an order of magnitude, for x-rays to propagate to the balloon from the lightning channels. The authors discussed the possibility of x-ray production in a high-field region just above the storm created temporarily by charge movements from a lightning flash. In this scenario, the pulse timescale of seconds derives from the relaxation time, the time it takes for a screening layer to reassemble and cancel the quasi-static field above the storm. Since this transient field was not detected at the balloon, the authors noted 


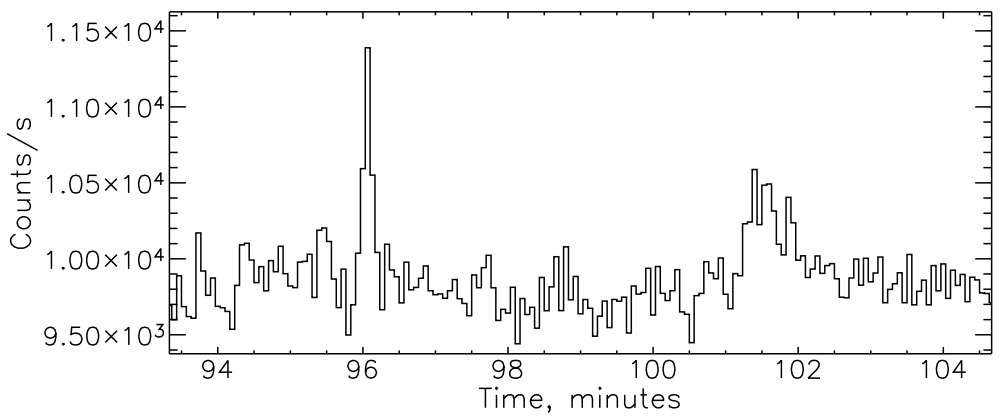

Fig. 12 Gamma-ray count rate for two glows observed by the ADELE instrument flying above two different active cells. The longer duration of the second event implies a greater spatial extent of the source

that the source would have to have been some distance away, implying very high x-ray intensities at the source. X-ray transients above the storm of $\sim 1$ second duration have not been observed again, and measurements confirming both the phenomenon and the explanation of its duration as representing the atmospheric relaxation time are needed.

In 2009, a set of gamma-ray detectors was flown on the Gulfstream V jet operated by NOAA for the NSF over and next to active thunderstorm cells in Florida. This instrument, the Airborne Detector for Energetic Lightning Emissions (ADELE), included 5" diameter by $5^{\prime \prime}$ long plastic and $\mathrm{NaI}$ scintillators for gamma-ray sensitivity above $10 \mathrm{MeV}$, a design based on the results of ground-based and satellite observations over the previous decade. Lead sheets placed above and below some of the detectors gave a crude sensitivity to whether radiation was incident from above or below.

ADELE observed one TGF during 37 hours aloft (Smith et al. 2011a), but observed glows on 12 separate occasions during passes over or near the tops of active cells (Kelley et al. 2010; see Fig. 12). In all the airplane data (Parks et al. 1981; McCarthy and Parks 1985) and even the balloon data (Eack et al. 1996a, 2000), it could always be either the motion of the detector's platform, or else a terminating flash, that limits the apparent duration of the event. It is quite possible that glows can be as long-lived as the high-field region in the storm producing them, but testing this requires longer data sets aloft and flight plans designed to sample the same cell repeatedly. The full ADELE data set, including spectral and directional information, is being evaluated now to test the hypothesis that these glows seen from above the cells indeed represent relativistic runaway, and, if so, to determine in what part of the cell the avalanche was taking place.

\subsubsection{Glows Observed from the Ground}

Despite the apparent simplicity of making measurements from the ground, positive results of high-energy radiation enhancements that were generally accepted actually came later than for airborne measurements. There were two difficulties: first, the emission due to the decay chain of radon washed out by rain, and second, the thick absorbing column of air between the ground and the high-field regions of storms at several km altitude. Suszcynsky et al. (1996) discuss the former issue and give a review of earlier attempts. Good spectroscopy allows bremsstrahlung from runaway electrons, which has a smooth spectrum extending to $10 \mathrm{MeV}$ or more, to be distinguished from the radioactivity of radon daughters, which has a structured spectrum consisting of nuclear lines and their Comptonized (Compton scattered) continuum and has virtually no emission above $2.5 \mathrm{MeV}$ (see, e.g., Fig. 3 of Brunetti et al. 2000). 
Fig. 13 Summed spectra of three glows observed by Tsuchiya et al. (2011) from their $\mathrm{NaI}$ (solid circles) and CsI (open circles) detectors. For comparison, the spectra of many summed TGFs from RHESSI and AGILE are shown. None of the spectra are corrected for the various responses of the instruments, but the similarity is obvious

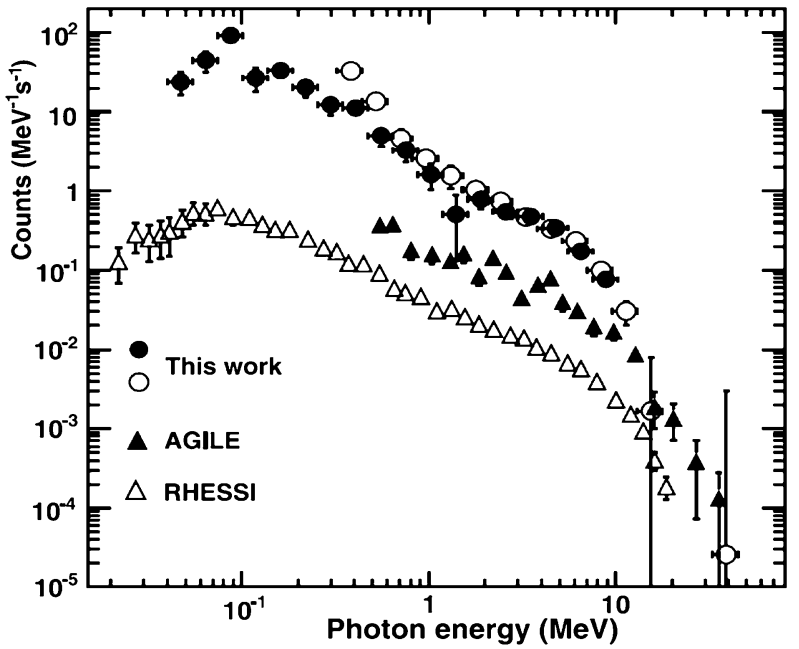

Recent measurements have demonstrated that there are two ways to overcome the problem of absorption in intervening atmosphere: by using a high-altitude site (Brunetti et al. 2000; Chubenko et al. 2000, 2003; Alexeenko et al. 2002; Torii et al. 2009; Tsuchiya et al. 2009; Chilingarian et al. 2010) and by studying storms where the charge centers are very low, specifically winter storms in Japan (Torii et al. 2002, 2008; Tsuchiya et al. 2007, 2011). It has been shown that in these locations, the phenomenon is common. For example, over 100 enhancements have been observed at the Aragats Space Environment Center, located at an altitude of 3,250 m, since 2003 (Chilingarian et al. 2010).

Brunetti et al. (2000) and Chubenko et al. (2000) made the first convincing measurements of x-ray/gamma-ray enhancements on the ground that were not related to radon washout. Brunetti et al. (2000) found events that extended in energy up to $10 \mathrm{MeV}$, beyond the range of nuclear decay gamma-rays, while Chubenko et al. (2000) emphasized the low-energy (mostly Comptonized) spectral component below $100 \mathrm{keV}$, but both groups recognized that both the energy spectrum and short duration ( $<5$ minutes) were consistent with the prediction of bremsstrahlung from runaway electrons and not radon washout. Torii et al. (2002) found the spectrum to be smooth and to extend beyond $5 \mathrm{MeV}$; more importantly, they pioneered the use in this field of sophisticated Monte Carlo simulations of high-energy electron and gamma-ray interactions in both the atmosphere and the detector to estimate the electron spectrum at the source. They found that the glow they observed on 1997 January 28 at the Monju nuclear reactor in Japan had a spectrum consistent with bremsstrahlung from electrons with energies of approximately 5-10 MeV. Torii et al. (2004) extended this modeling work. Similar analyses were performed by Tsuchiya et al. (2007, 2009, 2011), Torii et al. (2009), and Chilingarian et al. (2010) using data extending to even higher energies, with a similar conclusion. Tsuchiya et al. $(2007,2011)$ noted that the resulting spectrum was similar to that observed in TGFs by the RHESSI satellite (Smith et al. 2005) (see Fig. 13).

Both Brunetti et al. (2000) and Chubenko et al. (2000) as well as most later studies showed that glows seen from the ground have typical durations on the order 0.5-10 minutes. Menodonça et al. (2011) found a large sample of hour-scale radiation excesses during periods of precipitation at the CARPET cosmic-ray detector in Argentina. While these would typically be interpreted as radon washout in the absence of spectroscopic information, the authors noted that the radiation correlated with the presence of high electric fields when 
controlling for the total rainfall in the event, suggesting a possible role for particle acceleration even at these longer timescales. They used an array of Geiger counters with a $1 \%$ efficiency for counting gammas and a much higher sensitivity to charged particles, so the nature of the radiation is uncertain. A study of the typical size and speed of storm cells in the different geographical environments might be needed to reconcile these apparently very different time scales if the Argentine events are indeed due to runaway electrons like the usual minute-long events.

With the underlying spectrum well understood (Babich et al. 2010a), the degree of spectral distortion associated with Comptonization and photoelectric absorption has been used to determine the distance to the source (Tsuchiya et al. 2009, 2011). This requires both good counting statistics and a broad spectral response from $\sim 30 \mathrm{keV}$ to several $\mathrm{MeV}$ to capture the subtle spectral distortion caused by the atmosphere (see, e.g., Fig. 10 of Tsuchiya et al. 2011 or Fig. 2 of Dwyer and Smith 2005). Distances thus derived are typically less than $1 \mathrm{~km}$.

At $<\sim 100 \mathrm{~m}$ from the source, the primary accelerated electrons can be detected. Chubenko et al. (2003) required coincidences between multiple layers of Geiger-type ionization detectors to reject contamination by gammas, and demonstrated the direct detection of relativistic electron enhancements at the Tien-Shan Mountain cosmic ray station. Tsuchiya et al. (2009) also claim to have observed primary electrons in one glow event. They used an instrument in which a plastic scintillator covered a $\mathrm{NaI}$ scintillator, allowing only gammas to enter the NaI; but in that paper they did not report the expected number of gamma-ray interactions in the plastic scintillator, which could significantly contaminate the signal interpreted as electrons. Chilingarian et al. (2010) used thin plastic scintillators (for electrons) stacked on top of thick ones (for gamma-rays and very energetic charged particles) and explicitly simulated the cross-response of each detector to the type of radiation meant to be measured by the other. They found that their brightest event (2009 September 19) began with a gamma-ray signal and evolved into a mixed gamma-ray/electron signal, indicating that the acceleration region gradually enveloped the detector site over the 15-minute evolution of the event.

The observations of glows from the ground have often been made with instruments designed to study cosmic ray extensive air showers (Chubenko et al. 2000, 2003; Alexeenko et al. 2002; Chilingarian et al. 2010) or monitor radiation levels around nuclear reactors (Torii et al. 2002, 2008; Tsuchiya et al. 2011). These arrays are often large enough to constrain the size and motion of the glow source. Chubenko et al. (2000) showed that the glow emission seen on the ground extended to at least a few hundred meters, and Torii et al. (2002) showed that the extent was not much more than that ( $\sim 500 \mathrm{~m})$; this scale has been confirmed in later work (Chubenko et al. 2003; Chilingarian et al. 2010; Tsuchiya et al. 2011). Torii et al. (2002) first reported delays in peaking time from one detector to another (see Fig. 14) indicating motion of the source, and in later studies this sort of motion has been confirmed (Chubenko et al. 2003) and shown to roughly match the direction and speed of the prevailing wind (Tsuchiya et al. 2011). The duration of the glow might therefore be much greater than can be observed from one station or cluster of stations, with relative motion dominating the observed duration just as it does using airborne platforms. Some ground-level glows are clearly terminated by lightning, however (e.g., Alexeenko et al. 2002; Torii et al. 2008), as is also the case with some events seen from the air.

The enhancement of gamma-ray fluxes beyond the local background level is on the order of $10 \%$ for most glows. Count rates more than doubled during the 2009 September 19 event at Aragats, when a storm cloud was only 100-200 m above the detector (Chilingarian et al. 2010). But the brightest glow seen from the ground (Fig. 14) was the event reported by Torii 
Fig. 14 Count rate from $5 \mathrm{NaI}$ detectors arranged around the Monju nuclear reactor during a glow event in a winter thunderstorm (from Torii et al. 2002). Note the nearly hundredfold enhancement over background on the logarithmic scale (unique to this event), the variations in intensity from place to place constraining the size of the source region, and the shift in the peak time demonstrating motion of the source

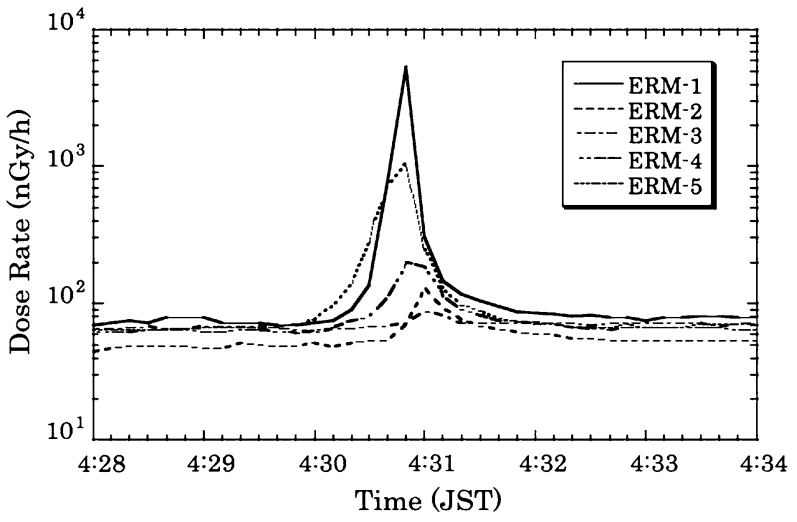

et al. (2002), at up to 70 times the local background level. This intensity is comparable to that seen by aircraft and balloons penetrating storms (Figs. 10 and 11) and indicates either that in this case particle acceleration was taking place near or at ground level, or that this event was intrinsically much more intense than is usually seen.

Determining the total high-energy electron content of a TGF from space requires only the observed intensity and the spectrally-derived atmospheric depth. Doing the same for gamma-ray glows observed from the ground requires knowledge of the source size as well, since they are being observed from a distance comparable to the size of the emitting region. Multi-detector arrays (to determine size) with good spectral response (to determine spectral distortion due to the atmosphere) are therefore necessary to calculate total electron content. The total electron content, combined with the size of the emitting region, gives the electron flux per square centimeter. The latter can also be measured directly if the detector is within the acceleration region itself. Either way, knowing the electron flux allows the most fundamental remaining physics question to be answered: how much of the theory developed in the last hundred years is necessary to explain glows seen from the ground? If faint enough, they could be consistent with simple acceleration of the ambient secondary cosmic-ray electrons (Wilson runaway). At higher luminosities it becomes necessary to invoke avalanche multiplication, and at even higher luminosity, relativistic feedback. Using gamma-ray intensity and a distance estimate derived from x-ray/gamma-ray spectroscopy for one event, Tsuchiya et al. (2011) estimated a total of $10^{9}$ to $10^{11}$ relativistic electrons involved over the course of a minute and an avalanche multiplication factor of 3-30. Chilingarian et al. (2010) used their direct detection of electrons on 2009 September 19 to constrain the distance even more precisely. They found an avalanche multiplication factor of $\sim 400$ ( $6 e$-foldings) relative to the known background of cosmic-ray secondary electrons, and a total electron number above $7 \mathrm{MeV}$ of $3.8 \times 10^{12}$ within the $\sim 500 \mathrm{~m}$ radius of the source. Since avalanche multiplication factors of up to $\sim 10^{4}$ can occur without feedback effects becoming important (Dwyer 2008), Tsuchiya et al. (2011) noted that feedback is not necessary to explain most glows seen from the ground.

The time profiles of some long-term enhancements in gamma-ray flux that might otherwise be associated with the radon decay chain have been studied, and have shown some anomalies at longer timescales than the glows described above (i.e., tens of minutes to hours) (Greenfield et al. 2003; Jayanthi et al. 2005; Salikhov et al. 2011). Greenfield et al. (2003) reported that these enhancements could appear when lightning but not rain was present, and in that case decayed with a half-life of about 50 minutes, longer than the $\sim 30$-minute 
half-life characteristic of the combined emissions of the radon daughters. These longerterm excesses have been interpreted as the decay of other isotopes produced in the thunderstorm by storm-accelerated protons or by cosmic-ray secondaries (Greenfield et al. 2003; Jayanthi et al. 2005). That hypothesis was not supported by nuclear spectroscopy, except in the case of a weak line at approximately $1.2 \mathrm{MeV}$ reported by Jayanthi et al. (2005), which they interpreted as a line of ${ }^{39} \mathrm{Cl}$ but which is also consistent with a line from the radon daughter ${ }^{214} \mathrm{Bi}$. Lundberg et al. (2011) performed high-resolution spectroscopy of long-term enhancements using a germanium detector, and found only lines from the radon daughters, and no signal in the ${ }^{39} \mathrm{Cl}$ lines. We note that, at present, no accepted mechanism exists for explaining how protons might be accelerated by thunderstorms or lightning.

Finally, direct observations of cosmic-ray secondary muons show that thunderstorm electric fields can either increase or decrease their flux slightly, depending on the sign of the field and the relative population of positive and negative muons (Alexeenko et al. 2002; Lidvansky 2003; Muraki et al. 2004).

\subsection{Short Duration X-Ray Emissions from Lightning}

\subsubsection{First Observation}

As of 2001, the existence of x-ray emissions from thunderclouds had been gaining support over the previous two decades, but the case for x-ray emissions from lightning remained weak, and so most researchers at the time did not accept that lightning generated $\mathrm{x}$-ray emissions. In 2001, Moore et al. reported the detection of energetic radiation associated with the lightning stepped leader phase of natural cloud-to-ground lightning on the mountains in New Mexico (Moore et al. 2001). At that time, it was not clear whether the energetic radiation was being produced by runaway electron production in the high fields associated with the leaders or by cosmic-rays seeding RREAs in the large scale electric fields.

\subsubsection{Detailed Investigations}

In a series of experiments beginning in 2002 at the University of Florida/Florida Tech International Center for Lightning Research and Testing (ICLRT) at Camp Blanding, FL, Dwyer et al. (2003) discovered that rocket-triggered lightning leaders emit bright bursts of energetic radiation (Dwyer et al. 2003; Krider 2003). Dwyer et al. (2004b) showed that the detected energetic radiation was predominantly x-rays with energies usually extending up to about $250 \mathrm{keV}$ and that the $\mathrm{x}$-rays were emitted in discrete bursts lasting less than $1 \mu$ s during the dart leader and dart-stepped leader phases and possibly at the beginning of the return strokes of negative triggered lightning. Dwyer et al. (2004b) also showed that X-ray emissions originated from the bottom few hundred meters of the leader channel and that the source propagated downward with the leader as it approached the ground.

Because the x-ray energies extend above $100 \mathrm{keV}$, thermal emission (not to be confused with thermal runaway) is ruled out as being the source since the maximum temperature occurring during lightning, $30,000 \mathrm{~K}$ during the return stroke (Uman 1984), is many orders of magnitude too low to account for such energetic x-ray emission. As a result, the only viable mechanism to explain this emission is through the production of runaway electrons in strong electric fields. In 2004, it was generally believed that RREA was responsible for the x-ray emissions from natural and triggered lightning. However, Dwyer (2004), using the Dwyer et al. (2004b) measurements, showed that the fluence and energy spectra of the $x-$ ray emissions from triggered lightning were not consistent with the RREA mechanism and 

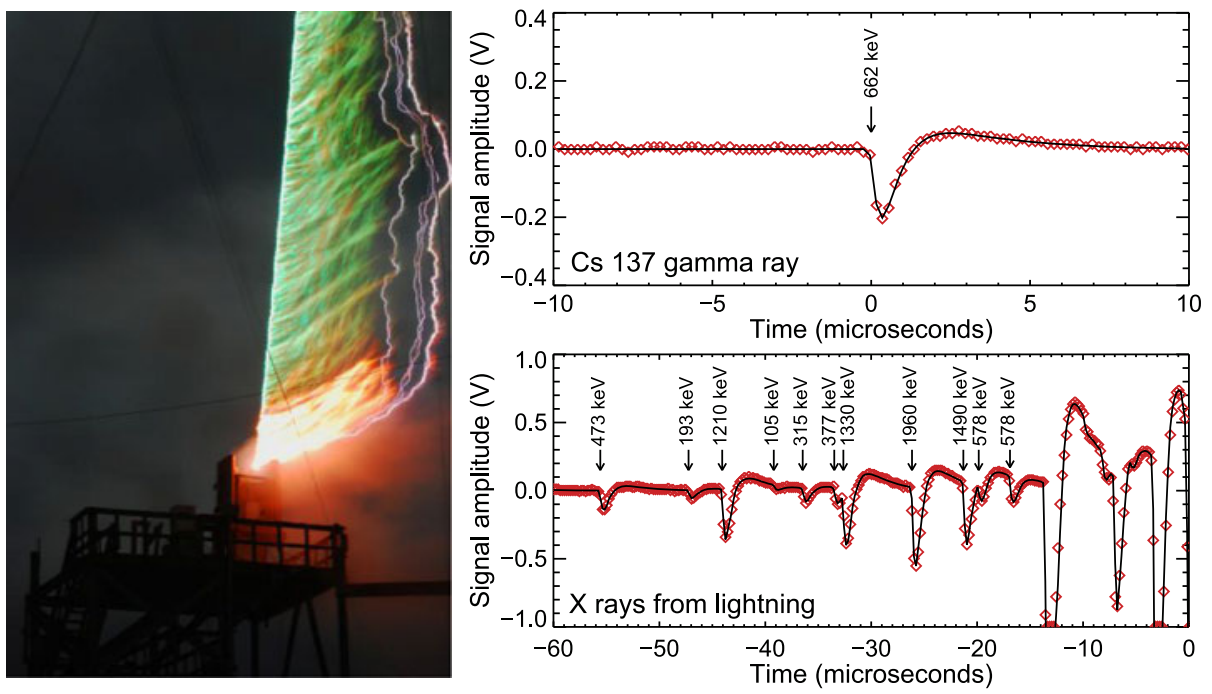

Fig. 15 Left: Photograph of rocket-triggered lightning at the ICLRT (courtesy Dustin Hill, UF). The illuminated straight triggering wire can be seen on the left. Four wind-blown leader/return stroke sequences are on the right. Top right: waveform from one of the $\mathrm{NaI}(\mathrm{Tl}) / \mathrm{PMT}$ detectors for a single $662 \mathrm{keV}$ gamma-ray from a Cs-137 radioactive source placed temporarily on top of the instrument. The red diamonds show the data as recorded by the acquisition system, and the solid line shows the detector response as calculated from the $\mathrm{NaI}$ light decay-time and the RC-times in the front-end electronics. Bottom right: waveform for a time period just prior to a return stroke (at $t=0$ ) of triggered lightning. The detector response (solid line) is plotted over the measured data (red diamonds). The arrows indicate the times and deposited energies of the x-rays. The figure is from Dwyer et al. (2004b)

proposed that instead the thermal runaway electron mechanism (Gurevich 1961) was responsible for the emissions. Later, Moss et al. (2006) modeled the thermal runaway electron production by streamers, and showed that significant runaway electron production was plausible. Other theoretical work includes Celestin and Pasko (2011), who also modeled thermal runaway electron production at streamer heads, Gurevich et al. (2007) who introduced a 2 step process (see Sect. 2.3 above), and Cooray et al. (2010) who modeled runaway electron production in the low-density, hot dart leader channel.

An example of the x-ray observations of triggered lightning is presented in Fig. 15, which shows the response of a $\mathrm{NaI}(\mathrm{Tl}) /$ photomultiplier tube (PMT) detector, housed in a heavy aluminum box to keep out RF noise, moisture and light. To illustrate the response of the instrument to x-rays, the top right panel of Fig. 15 shows the signal from one $662 \mathrm{keV}$ gamma-ray from a Cs-137 radioactive source placed temporarily on top of the instrument. The solid black curve shows the fit of the response function as derived from the electronics and the $0.23 \mu \mathrm{s} \mathrm{NaI}$ decay-time. The bottom right panel of Fig. 15 shows x-rays from rockettriggered lightning measured during the dart-stepped leader phase. In the figure, the bright return stroke occurred to the right at time $t=0$. The solid black curve is the fit of the response functions with the X-rays' deposited energies and times indicated by the arrows.

Saleh et al. (2009) used the Thunderstorm Energetic Radiation Array (TERA) at the ICLRT to make detailed measurements of the x-ray luminosity versus radial distance from the triggered lightning channel and used Monte Carlo simulations (REAM) to infer properties of the energetic electrons created by the lightning that generated the $\mathrm{x}$-rays. This paper presented an analysis of the $\mathrm{x}$-ray emission of three flashes. The $\mathrm{x}$-ray emission was ob- 


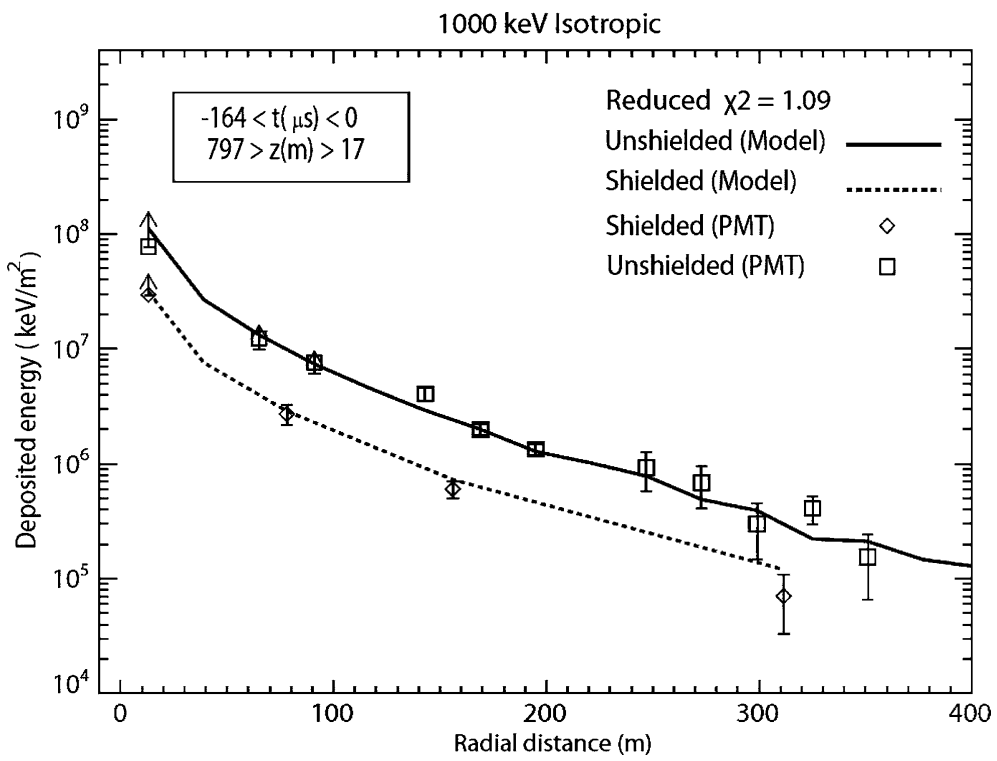

Fig. 16 X-ray intensity versus radial distance from the triggered lightning channel as measured by TERA at the ICLRT. The model fits from detailed Monte Carlo simulations are also shown. The model is for energetic runaway electrons emitted isotropically from the lightning leader with a characteristic energy of $1 \mathrm{MeV}$. The shielded detectors are covered with $0.32 \mathrm{~cm}$ of lead. From Saleh et al. (2009)

Fig. 17 Energetic runaway electron luminosity from lightning versus altitude above the ground as measured at the ICLRT. From Saleh et al. (2009)

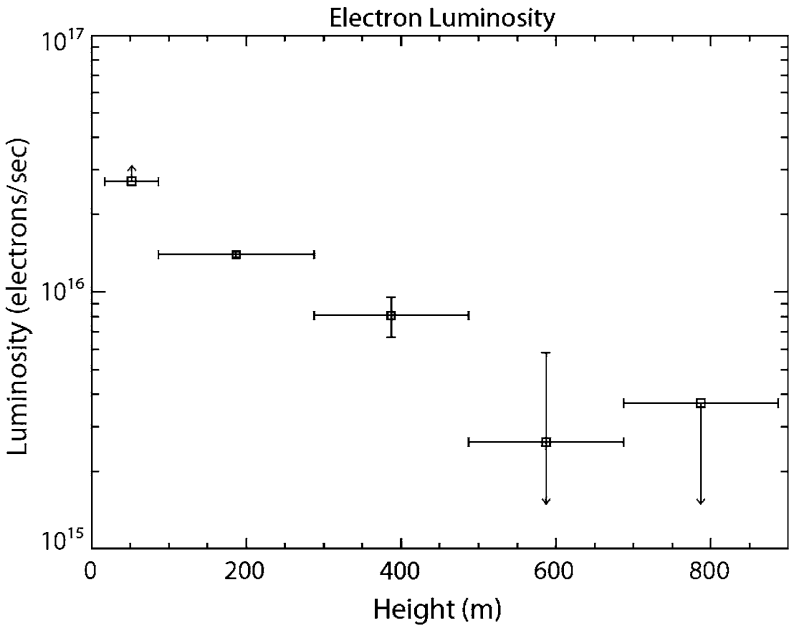

served to occur during the dart-stepped leader phase of each stroke, just prior to the time of the return stroke. Significant X-rays were observed on all the detectors out to a distance of $500 \mathrm{~m}$ from the triggered lightning channel for times up to $200 \mu \mathrm{s}$ prior to the start of the return stroke. Using Monte Carlo simulations to model the x-ray propagation, they found that the energetic electrons that emit the x-rays had a characteristic energy of about $1 \mathrm{MeV}$ for one particular dart-stepped leader event. The x-ray emission for all three events has a radial fall off most consistent with the energetic source electrons being emitted isotropically from the leader (see Fig. 16). It was also found that the x-ray and energetic electron luminosities of the leader channel decrease with increasing height above the ground (Fig. 17). Of particular 
importance was that TERA measured, for the first time, the luminosity of energetic electrons [electrons/second] from triggered lightning. It was found that the luminosity exceeded $10^{16}$ electrons/s near the ground. This luminosity is large enough that the emission of x-rays from lightning is a good candidate for the RREA seed particles of terrestrial gamma-ray flashes (TGFs) inside thunderclouds, possibly unifying the two phenomena (Dwyer et al. 2010).

The propagation mechanisms of lightning are generally not well understood. Negative lightning leaders, particularly those of the stepped and dart-stepped types, do not propagate in a continuous manner, but instead progress in a series of discrete "steps." For stepped leaders, individual steps often have step lengths from 10-50 m and typical inter-step intervals of some tens of $\mu$ s (Rakov and Uman 2003). Stepped leaders are typically characterized by significant branching and tortuosity, while dart-stepped leaders typically follow the pre-conditioned path of a previous leader/return stroke. In order to understand how lightning propagates, it is critical to understand how stepped leaders form. Laboratory experiments show that negative leaders produce $(\sim 1 \mathrm{~m}$ or less) steps by creating a plasma channel in the volume in front of the old leader channel, called a space stem (Bazelyan and Raizer 1998). High-speed video observations (Biagi et al. 2010; Hill et al. 2011) show that space stems also form for triggered lightning dart-stepped leaders and natural lightning stepped leaders, demonstrating that the space stem mechanism of propagation is similar to that in long laboratory sparks. However, it is not clear how the observed leader propagation mechanisms and the underlying physics change with the vastly different physical scales of the discharges. X-ray measurements of the step formation processes in natural and triggered lightning and laboratory sparks may help answer this question.

Dwyer et al. (2005b) showed that x-ray bursts detected during natural cloud-to-ground lightning at the ICLRT were produced by the stepped leader during the formation of the steps, indicating a connection between the x-ray emission and lightning leader propagation. Figure 18 shows the $\mathrm{x}$-ray pulses emitted by the stepped leader. The $\mathrm{x}$-ray emission from the stepped leaders are surprisingly similar to the x-ray emission recorded during dart and dartstepped leaders in triggered lightning. This result was extended by Howard et al. (2008). Using a time of arrival technique of the x-ray pulses using an 8 station subset of TERA, they showed that the $\mathrm{x}$-ray emissions from both triggered lightning and natural lightning are spatially co-located with the leader step formation, establishing a physical link between the two phenomena. As a result, $\mathrm{x}$-rays can help determine the spatial structure of the stepped leaders. For instance, the emission angles of the x-rays help map out the electric field lines near the lightning. In addition, by analyzing x-ray data from TERA in conjunction with data from the co-located $d E / d t$ sensors, Howard et al. (2010) discovered that a commonly occurring burst of $d E / d t$ pulses immediately prior to the return stroke, called "leader burst pulses," are one of the brightest sources of x-ray from lightning. These pulses, identified in $d E / d t$ data for natural and triggered lightning, appear different from stepped leader and dart-stepped pulses and seem to be part of the attachment process, taking place within tens of meters from the ground. In addition, Hill et al. (2012) reported the first observations of unique energetic radiation emissions from chaotic dart leaders preceding triggered lightning return strokes. In four events, a relatively continuous flux of energetic radiation was observed during the final 10-13 $\mu$ s of the descending leader from altitudes of several hundred meters, determined from $d E / d t$ time-of-arrival (TOA) measurements. Other observations of x-ray emissions from natural lightning include Yoshida et al. (2008), who reported the only detection of x-rays from a positive lightning leader.

Dwyer et al. (2011) made the first high-time-resolution two-dimensional images of the $\mathrm{x}$-ray emissions from triggered lightning. The images were recorded at a rate of 10 million frames per second using a new pinhole-type camera, located $44 \mathrm{~m}$ from the triggered 
Fig. 18 X-rays from NaT/PMT detectors (top panel) and electric field waveforms (bottom panel) for a natural cloud-to-ground lightning flash at the ICLRT. Time zero in the plot corresponds to the beginning of the return stroke. The step formation times are denoted by vertical dotted lines. As can be seen the X-ray pulses are closely associated with the step leader formation. From Dwyer et al. (2005b)
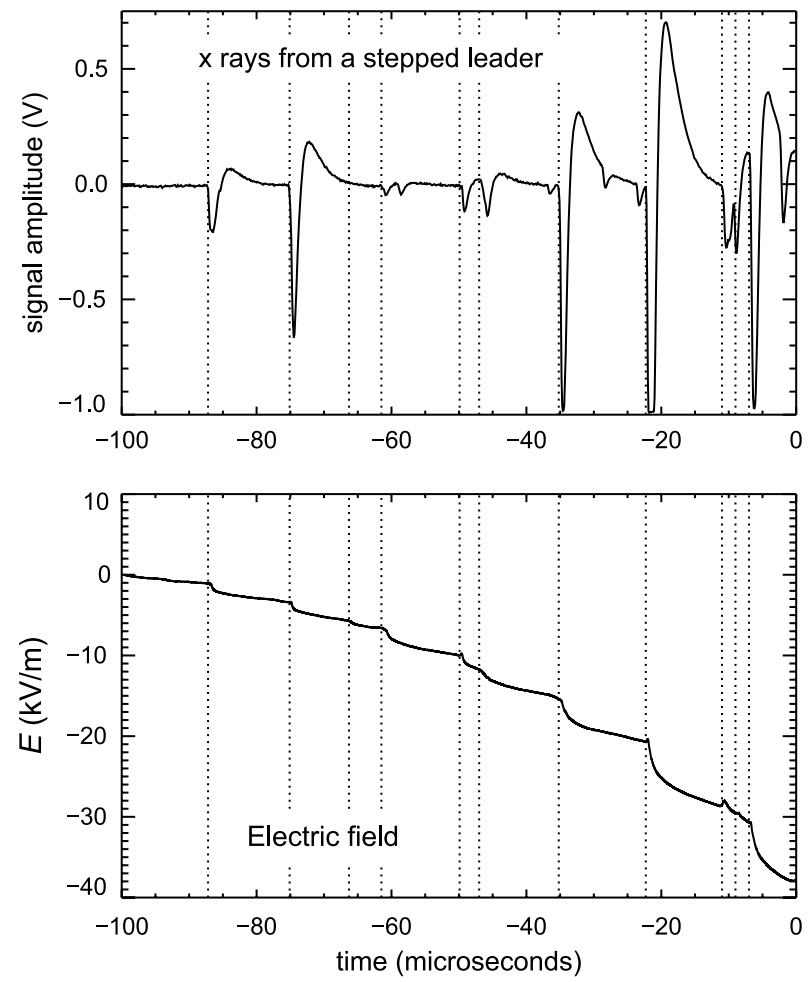

lightning channel. They observed two chaotic dart leaders, one in each of two lightning flashes triggered during the summer of 2010 at the ICLRT. In both events, as the chaotic dart leader approached the ground, the x-ray source was also seen to descend along the previous lightning channel. For the second event, the x-ray source exhibited a downward speed of $4.5 \times 10^{7} \mathrm{~m} / \mathrm{s}$, in agreement with independent $d E / d t$ time-of-arrival measurements of the speed of the leader front, demonstrating that the leader front was the source of the X-ray emission. The camera also recorded bursts of $\mathrm{MeV}$ gamma-rays originating from the chaotic dart leader and/or the ground attachment process of the leader. Figure 19 shows a sequence of $\mathrm{x}$-ray images of a rocket triggered lightning leader as it approached the ground, showing that most of the $x$-ray emission originates from the tip of the descending leader. Because the background rate is very low, virtually all the non-black pixels seen in Fig. 19 correspond to $\mathrm{x}$-rays emitted by the lightning leader.

A number of observations have been made at the Tien Shan High-Altitude Scientific Station of the Physical Institute of the Academy of Sciences (Antonova et al. 2007; Chubenko et al. 2000, 2003, 2009; Gurevich et al. 2004c, 2009a, 2009b, 2011a). This facility, which is located between 3.4 and $4 \mathrm{~km}$ above sea level, has the capability of recording RF signals, gamma-rays and cosmic-ray air showers. Antonova et al. (2009) reported the association of short (100 ns) RF pulses that arrive in coincidence with extensive air showers (EASs) during thunderstorms, possibly resulting from RREA multiplication acting on the EASs. Gammarays were also reported before the return strokes of natural lightning. Gurevich et al. (2009a) reported an interesting event in which their EAS trigger coincided with a strong gamma-ray pulse followed by an abrupt decrease in the background gamma-ray flux. They interpreted 


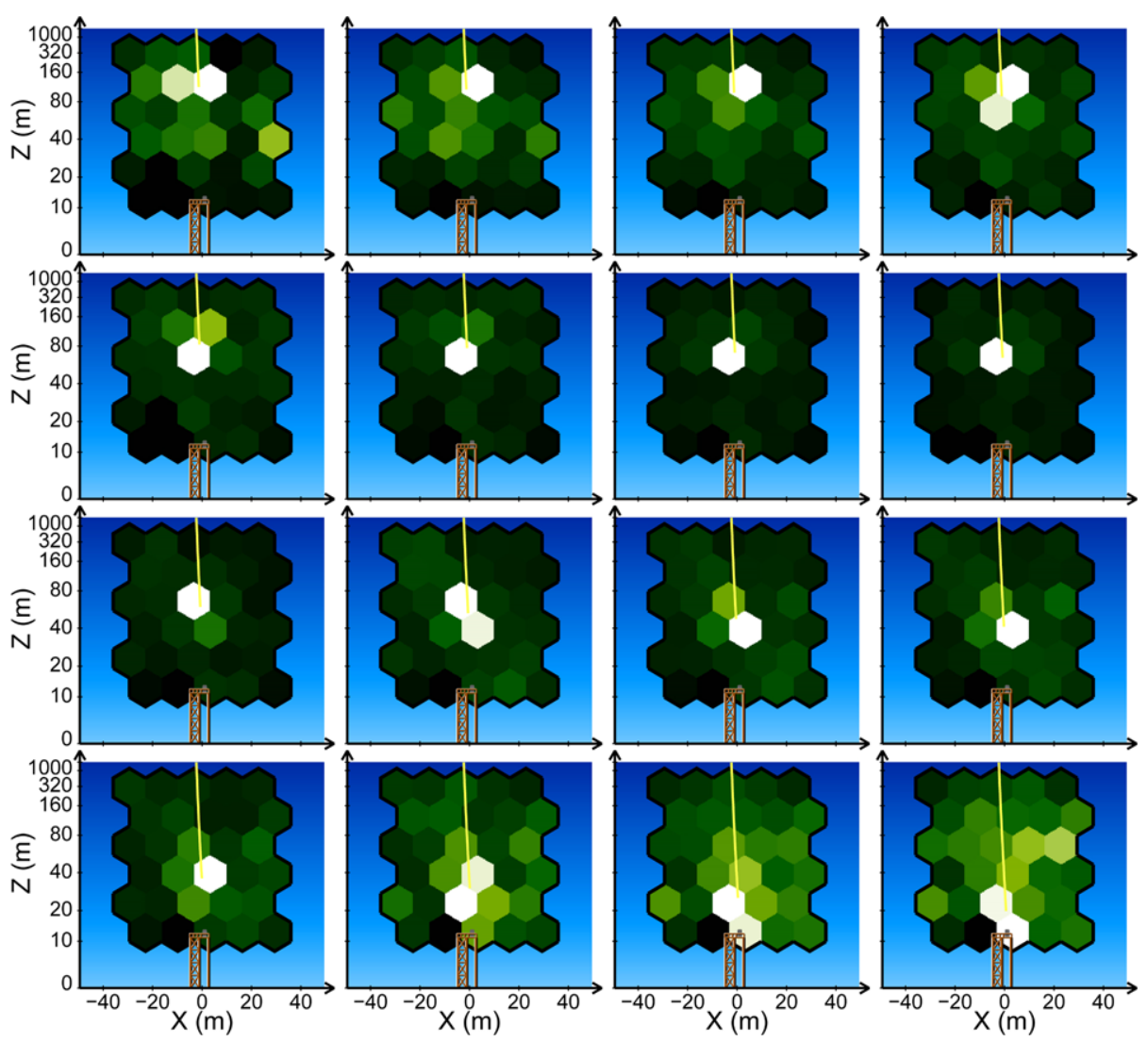

Fig. 19 Successive $0.1 \mu$ s images of the x-ray emission from lightning, recorded on August 13, 2010 at the ICLRT. The first image is in the upper left corner and time progresses from left to right. The exposures cover the observation times from $-1.53 \mu$ s to $+0.07 \mu$ s relative to the return stroke. This corresponds to the emission times from $-1.94 \mu$ s to $-0.087 \mu$ s before the return stroke. The rocket launch tower is illustrated at the bottom along with the approximate location of the lightning channel. The color scale for each image has been adjusted so that the detector with the maximum deposited energy appears as white. This maximum deposited energy is $5.5 \mathrm{MeV}$ in the first image, increases as the lightning approaches the ground, reaching a maximum of $28.2 \mathrm{MeV}$ in the eleventh image, and then decreases to $16.6 \mathrm{MeV}$ in the last image. Note that the deposited energy in each detector corresponds to the detection of multiple x-ray photons. From Dwyer et al. (2011)

their data as indicating that the EAS caused a discharge within the thundercloud. Finally Gurevich et al. (2011a) reported gamma-ray emissions lasting hundreds of msec in association with thunderstorms. Their measurements showed a puzzling altitude dependence, with detectors separated by a few hundred meters (at $>3 \mathrm{~km}$ altitude) showing orders of magnitude decrease in recorded gamma-ray flux.

\subsubsection{Role of Runaway Electrons in Lightning Processes}

It is not clear how important the runaway electron and x-ray productions are for the propagation of natural and triggered lightning. It is conceivable that the runaway electrons modify the conductivity of the air in a way that affects the propagation, or that the x-rays provide low energy seed elections for conventional avalanche multiplication. However, neither of 
these scenarios have been established. It is possible that the high-energy emission is simply an interesting side effect that does not influence the lightning properties substantially.

If we use the result from Dwyer et al. (2010) that each triggered lightning leader step produces about $10^{11}$ energetic runaway electrons with an average energy is about $1 \mathrm{MeV}$ (Saleh et al. 2009), then these will generate about $10^{11} \times 10^{6} \mathrm{eV} / 34 \mathrm{eV}=3 \times 10^{15}$ low-energy electrons, where $34 \mathrm{eV}$ is the $W$-value for air, giving the average energy required to generate a low-energy electron-ion pair. The resulting current is highly model dependent, sensitive to the electric field and pulse duration (since electron attachment times are important), but it is conceivable that these low-energy electrons could produce a brief current pulse on the order of a hundred amps. This is about an order of magnitude smaller than the peak current measured for step leaders during the step formation process (Rakov and Uman 2003). The leader steps are also known to propagate by forming streamers and hot conductive channels, so it is clear that the runaway electron generation is not the entire story as calculations by some authors seem to suggest (Milikh and Roussel-Dupré 2010).

Perhaps a better way to look at the problem is that the energetic electron production cannot be separated from the low-energy electron population and that the entire electron distribution should be taken as a whole. In other words, it is desirable to understand the electron distributions that occur during lightning propagation and these distributions cannot be fully described without including the energetic particles. Finally, at the very least the $\mathrm{x}$-ray production is providing a new tool for studying lightning as the $\mathrm{x}$-ray camera images illustrate.

\subsection{Short Duration X-Ray Emissions from Long Laboratory Sparks in Air}

Although there is large body of work describing the x-ray emission in small $(<\mathrm{few} \mathrm{cm})$, highly stressed gaps (see Babich 2003 for a review of this literature), before 2001 it was generally believed that electrical discharges in air involved only low-energy electrons having energies of at most a few tens of eV (Raether 1964; Bazelyan and Raizer 1998). The discovery that both natural and triggered lightning discharges emit x-rays (Moore et al. 2001; Dwyer et al. 2003) demonstrated that some kinds of discharges in air produce high-energy electrons traveling close to the speed of light and having energies of at least hundreds of keV. At the time, the x-ray observations of lightning seemed to support the generally-accepted notion that lightning was significantly different from laboratory sparks, the latter being assumed to involve only conventional breakdown with no runaway electrons production as with lightning. In 2005, Dwyer et al. (2005a) made the surprising discovery that long laboratory sparks in air at 1 atmosphere pressure also emit x-rays, very similar in characteristics to lightning discharges. Specifically, using a 1.5 MV Marx generator they found that both positive and negative polarity sparks, with lengths ranging from about $10 \mathrm{~cm}$ up to 2 meters generated $\mathrm{x}$-ray pulses in the hundred keV range. These $\mathrm{x}$-rays were usually produced when either the voltage was near its peak value or the voltage in the gap was in the process of collapsing. This work has since been confirmed and expanded upon by several groups in different high voltage laboratories (Dwyer et al. 2008a; Rahman et al. 2008; Nguyen et al. 2008, 2010; March and Montanyà 2010, 2011; Gurevich et al. 2011b). Like lightning, the energy spectra of x-rays from laboratory sparks is too soft to be produced by RREA, which, as we discussed in Sect. 2.2.4, has an average energy of $7 \mathrm{MeV}$, indicating a different mechanism for the production of the runaway electrons (Dwyer 2004). Furthermore, the fact that copious x-rays are produced with voltages down to about $0.5 \mathrm{MV}$, rules out the RREA mechanisms, which requires many tens of $\mathrm{MeV}$ in order to generate substantial numbers of runaway electrons. Theoretical work, using the thermal runaway electron 
mechanism, includes Gurevich (1961), Moss et al. (2006), Gurevich et al. (2007), Li et al. (2009), Chanrion and Neubert (2008, 2010), Cooray et al. (2009), Colman et al. (2010), and Celestin and Pasko (2011).

\subsection{Neutrons}

\subsubsection{Observations of Neutrons from Thunderstorms}

Several groups have reported neutron excesses associated with thunderstorms. The earliest search for neutrons in natural lightning was by Fleischer (1975), using dosimeters placed on lightning rods. This work produced only upper limits. Shah et al. (1985) made the first positive detections, estimating $10^{7}-10^{10}$ neutrons per stroke, a range consistent with the upper limits of Fleischer (1975). Shah et al. (1985) achieved low-background observations by triggering a short window for neutron data acquisition on the electric field change associated with a lightning flash. Shyam and Kaushik (1999) took an opposite approach. Suspecting that large cosmic ray showers might trigger lightning, with the neutrons coming from the shower itself rather than the lightning discharge, they compared long time intervals during thunderstorms with background intervals that presumably contain the same frequency of cosmic ray showers. They found a $4.8 \sigma$ excess of neutron counts during the times with nearby lightning.

More recent ground-based observations were made by Kuzhevskii (2004), Chilingarian et al. (2010), and Martin and Alves (2010). Kuzhevskii (2004) observed several events from a station in Moscow. While the author describes these events as being associated with lightning flashes per se, the data (Figs. 1 and 2, Kuzhevskii 2004) clearly show that the neutron excesses are on the order of a minute long, and therefore are more likely to be related to gamma-ray glows. The lightning flashes that the author associates with these events may have terminated or commenced the glows (see Sect. 3.2.1). Martin and Alves (2010) recorded a single event in Brazil in which a ${ }^{3} \mathrm{He}$-filled, pressurized proportional counter serving as a neutron detector, integrating 1-minute samples, registered a single sample during a thunderstorm of 690 neutrons (in comparison to a background of less than 1 count per minute). A nearby lightning strike occurred during this minute. The authors estimated that the number of neutrons produced was $10^{12}$ to $10^{13}$ at the lightning channel, $0.5 \mathrm{~km}$ from the detector. Unlike the observations of Kuzhevskii (2004), this observation does not allow us to determine whether the excess was extremely short or nearly a minute long, since only a single sample was high. Chilingarian et al. (2010) report a neutron excess during a bright gamma-ray glow of 2009 September 19, with duration $\sim 10$ minutes. While the neutron events seen by Kuzhevskii (2004) and Martin and Alves (2010) were more than an order of magnitude higher than the background levels in their instruments, the neutron event reported by Chilingarian et al. (2010), shown in their Fig. 3, is only a $3 \%$ excess over background. Still, this represents the only simultaneous report of neutrons and a gamma-ray glow.

Excesses of neutrons, in the form of discrete bursts, have also been observed from orbit with instruments on the MIR space station and the Kolibri-2000 satellite, with a longitude distribution peaking over Africa and the Pacific (Bratolyubova-Tsulukidze et al. 2004). Individual storms were not identified with the neutron bursts in this work, and the MIR events appear to have a latitude distribution less equatorial than that of lightning. A new space instrument is planned specifically to study these events (Drozdov et al. 2010).

\subsubsection{Interpretation of Neutron Observations}

For most of the time since their discovery, it was assumed that neutrons associated with lightning were created in nuclear reactions, primarily deuterium/deuterium fusion. This idea 
goes back to before the discovery was even made, when Libby and Lukens (1973) suggested lightning-generated neutrons as an explanation for anomalies in the radiocarbon dating of tree rings. But Babich (2006) calculated the neutron yield of lightning to be negligibly small by this mechanism, even for unrealistically high fields of $3 \mathrm{MV} / \mathrm{m}$. Both the scarcity of deuterium in the atmosphere and low expected energies for the ions contributed to the conclusion that the neutron yield from a lightning flash from fusion would be $\ll 1$ particle. In the same paper, Babich (2006) introduced the alternate mechanism of photoproduction of neutrons when a gamma-ray generated in a TGF collides with a nucleus. The gamma-ray energy threshold for, e.g., ${ }^{14} \mathrm{~N}$ is $10.55 \mathrm{MeV}$, well below the maximum seen in TGFs (see Sect. 4). For gamma-rays above this energy, the neutron yield has been calculated as $0.43 \%$ (Babich et al. 2010b). Babich (2006) estimated that the neutron yield of a TGF would be $10^{15}$ for a TGF producing $10^{17}$ relativistic electrons. Further modeling has supported the conclusion that neutron photoproduction is significant (Babich 2007; Babich and Roussel-Dupré 2007; Babich et al. 2007a, 2007d, 2008a), although Carlson et al. (2010b), using GEANT4 Monte Carlo simulations, found a lower yield of $10^{12}$ neutrons in a TGF with $10^{16}$ energetic photons. Carlson et al. (2010b) note that only $\sim 1 \%$ of TGF gamma-rays have sufficient energy for neutron photoproduction. Their results include the neutron spectrum and arrival time distribution at the ground and on orbit for comparison with the known observations. They concluded that a downward-directed TGF of normal luminosity at an altitude of $5 \mathrm{~km}$ or lower might reproduce the neutron observations. However, they also found that the neutron flux in Earth orbit from an upward TGF was far too small to explain the events seen by Bratolyubova-Tsulukidze et al. (2004). Babich et al. (2010b) estimated neutron production for runaway avalanches in the high-field volume of a thunderstorm. While not explicitly calling this a simulation of a TGF, the simulated event duration $(<3.5 \mathrm{~ms})$ and derived gamma-ray flux suggest that this is essentially also a downward-TGF model. They found that a source at $8-12 \mathrm{~km}$ could produce a neutron flux at a mountaintop observatory at $3 \mathrm{~km}$ of the order of that seen by Shah et al. (1985).

It is worth pointing out that none of the observations of neutron excesses has definitively shown a short duration implying that the source is lightning or a TGF. They have either explicitly shown a duration comparable to gamma-ray glows (Kuzhevskii 2004; Chilingarian et al. 2010), or else the data were taken in a way that doesn't distinguish between the two time scales (Shyam and Kaushik 1999; Martin and Alves 2010). The case of Shah et al. (1985) is the most complicated in this regard. They recorded the total neutron counts in a 320 microsecond window beginning at the collection time of the first neutron detected after an antenna triggered the presence of a nearby lightning strike. Many of the neutron excesses were statistically significant, but also occurred more than a millisecond after the triggering stroke, with a few over 100 milliseconds later. The authors attributed these events to either subsequent strokes from the triggered stroke or very slow neutrons, but a re-interpretation of this work in which the neutron-detection window is opened at the start of or in the midst of a glow may be a worthwhile project. At any rate, new observations that sample continuously at very high time resolution would be the clearest way to determine if neutrons are associated only with glows or with lightning as well. None of the theoretical papers to date discuss neutron production from glows produced over long time periods and very near to the source, although, at least in the case of Chilingarian et al. (2010), this is obviously the physical situation: the glow for which they observed the neutrons was so close to their mountaintop detectors that the freshly accelerated electrons themselves were also observed.

The possible sensitivity of the various neutron detectors to gamma-rays has not been sufficiently addressed. Only Chilingarian et al. (2010) used multiple instruments with different relative sensitivities to neutrons and gammas; however, the response of the neutron-specific 
detector to gammas was not calculated in this paper, it was only stated to be negligible. Explicit calculations or simulations of the sensitivity of all neutron instruments to gammarays, and the use of primarily gamma-sensitive detectors in parallel with primarily neutronsensitive ones, should be features of all neutron-detection work in order to unequivocally separate neutron and gamma signals.

There does not, at the moment, seem to be a theory that would explain the production of neutrons in CG lightning. While leaders produce bursts of x-rays, these have not been seen to have the significant emission above $10 \mathrm{MeV}$ necessary for photoproduction (see Sect. 3.3). Carlson et al. (2010b) note that neutrons seen from the ground due to a downwarddirected TGF should be accompanied by a detectable level of gamma-rays above $10 \mathrm{MeV}$, a phenomenon that has only been observed once, in triggered lightning (Dwyer et al. 2004a).

\subsection{Laboratory Studies of RREA}

Because RREAs require very large potential differences, e.g. $>7 \mathrm{MV}$ per avalanche length, it is difficult to study RREAs in laboratories. For this reason, most of our knowledge about RREAs comes from simulations and the observations of thundercloud, which can produce very large potentials. On the other hand, the study of thermal runaway electron production is possible in the laboratory during, for example, long sparks (see Sect. 3.4). Some groups have attempted to investigate either the early stages of RREA development (Babich et al. 2002, 2004b). Other groups have used trapped electrons and the microwave electron cyclotron resonance to study RREAs in the lab (Gurevich et al. 2001a; Sergeichev and Sychev 2002). For these latter experiments, because the resulting energetic emission generated was below $300 \mathrm{keV}$, it is not obvious that these energetic particles were produced by the RREA mechanism, which has an average energy of $7 \mathrm{MeV}$.

\section{Terrestrial Gamma-Ray Flashes (TGFs)}

\subsection{Observations of Terrestrial Gamma-Ray Flashes: The BATSE Era}

Terrestrial gamma-ray flashes (TGFs) were first discovered in data from the Burst and Transient Source Experiment (BATSE) aboard NASA's Compton Gamma-ray Observatory (CGRO), one of the series of Great Observatories that includes the Hubble Space Telescope. BATSE's primary science target was cosmic gamma-ray bursts (GRBs), and from the BATSE data we learned that these are an extragalactic phenomenon. Ironically, GRBs themselves were first discovered by spacecraft intended to detect gamma-ray flashes from below, as an indication of atmospheric nuclear tests (Klebesadel et al. 1973). BATSE (Fishman et al. 1989) consisted of eight sets of scintillators, one set facing outwards from each corner of the spacecraft, for sensitivity to x-rays and gamma-rays from the whole sky. Since $C G R O$ was in low-Earth orbit, the surface below was always visible to four detectors. The Large Area Detectors (LAD) were sheets of NaI $1.27 \mathrm{~cm}$ thick with 2,025 $\mathrm{cm}^{2}$ of effective area. Each module also had a spectroscopy detector (SD) in the form of a cylinder of NaI $7.6 \mathrm{~cm}$ thick and $12.7 \mathrm{~cm}$ in diameter. The literature on BATSE TGFs uses only the LAD data, but the SDs saw the TGFs as well.

Figure 20, from the TGF discovery paper (Fishman et al. 1994), shows time profiles of TGFs as seen by BATSE. The typical duration is on the order of a millisecond, as opposed to GRB durations, which are typically a few seconds. Varying pulse widths, and sometimes multiple pulses, are seen. BATSE was a triggered instrument, returning short intervals of 

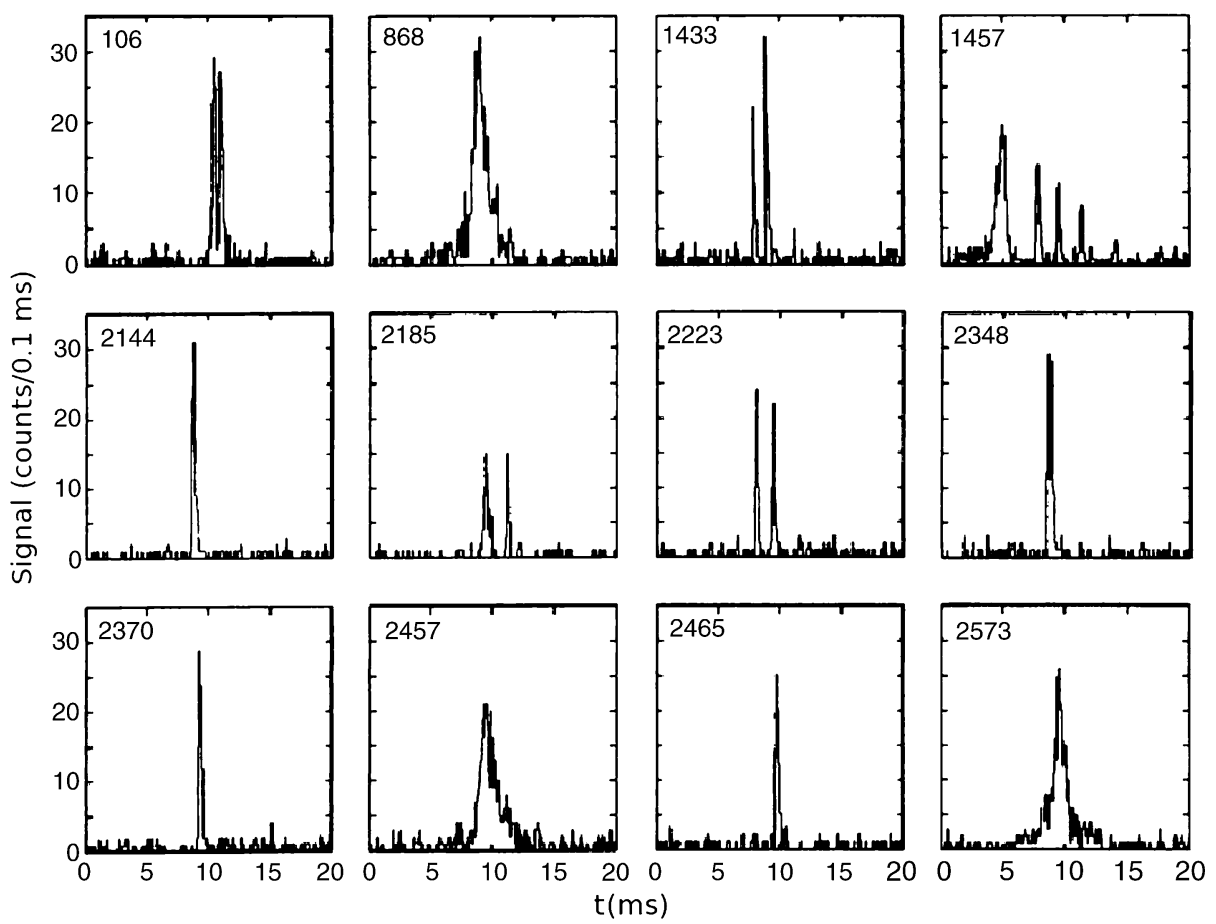

Fig. 20 Time profiles of terrestrial gamma-ray flashes (TGFs) observed by BATSE. From Fishman et al. (1994). Reprinted with permission from AAAS

high-time-resolution data when an onboard algorithm detected a sharp increase in count rate above background on time scales down to $64 \mathrm{~ms}$. This time-tagged event (TTE) format gave the arrival time of each count in the LADs to $2 \mu$ s accuracy, but expressed the deposited energy as one of only four channels, approximately $20-50 \mathrm{keV}, 50-100 \mathrm{keV}, 100-300 \mathrm{keV}$, and $>300 \mathrm{keV}$. Fishman et al. (1994) noted that the long trigger window meant that weaker events would not be seen as significant over $64 \mathrm{~ms}$ worth of background counts, and that many more fainter TGFs might have been missed.

Fishman et al. (1994) indicated that TGFs were associated with thunderstorms, and shortly afterwards Inan et al. (1996) discovered that individual radio atmospherics ("sferics"), the signature of a lightning flash, were associated with TGFs. For the first decade after their discovery, TGFs were generally assumed to originate from high-altitude runaway electron production, most likely associated with sprites, luminous high altitude discharges that had been discovered a few years earlier. Later discoveries overturned this presumed association (see Sect. 4.4 below): In 2004, Dwyer et al. (2004a) reported a gamma-ray flash observed on the ground in Florida in association with rocket-triggered lightning. The event, which seemed to have originated from the overhead thundercloud, had an energy spectrum and duration similar to TGFs. Dwyer et al. suggested that a similar thundercloud event that was directed upward might explain TGFs.

Substantial but still incomplete understanding of the lightning processes responsible for TGFs has come from detailed analysis of the radio emissions associated with TGF production, which is described in Sect. 4.4. The study of associated lightning is our best hope of understanding the details of TGF physics, since the gamma-ray spectrum of relativistic run- 
Fig. 21 Relativistic runaway electron avalanche (RREA) spectra at different altitudes compared to the RHESSI spectrum of 289 summed TGFs. The atmospheric depths from 13 to 130 grams per square centimeter correspond to 30, 24, 21 , and $15 \mathrm{~km}$. From Dwyer and Smith (2005)

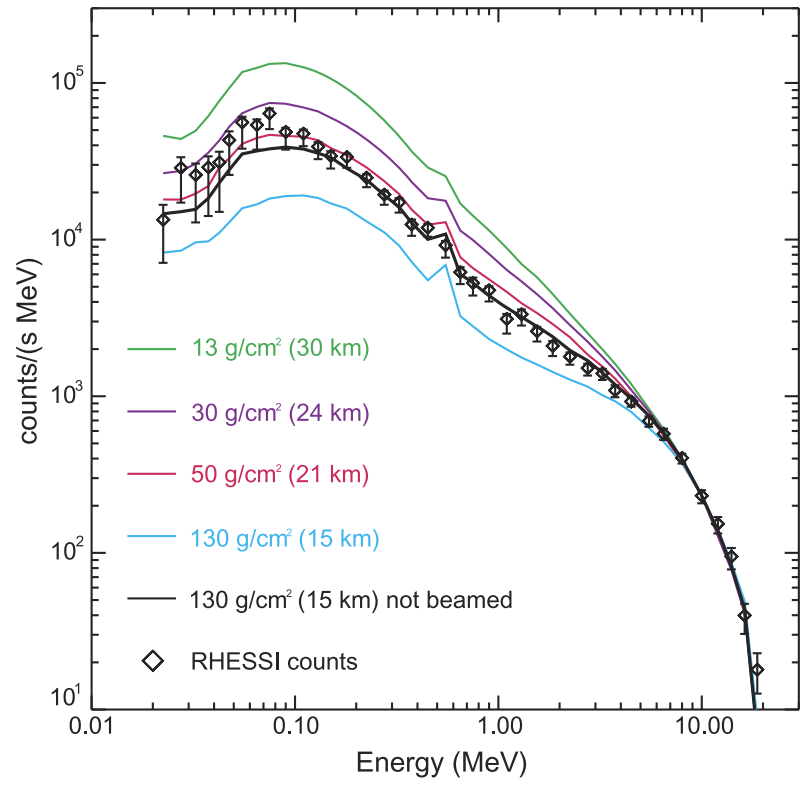

away electrons is not highly sensitive to electric field strength, total voltage, or the size or nature of the production region.

The spectra of TGFs were known from the start to be much harder than those of GRBs, other cosmic sources, and solar flares (Fishman et al. 1994). Nemiroff et al. (1997) and Feng et al. (2002) performed more detailed analyses of the BATSE data, looking at the temporal and spectral variations, and noting that softer photons (below $100 \mathrm{keV}$ ) tend to arrive later. Nemiroff et al. (1997) noted that TGFs with a softer spectrum tend to have faster characteristic timescale variations, a result that should be explored in reference to more recent data. They also noted that the spectral asymmetry in time rules out any models in which the short duration is caused by a beam sweeping across the spacecraft. Østgaard et al. (2008) and Grefenstette et al. (2008) associated this softening with the presence of a delayed tail due to photons that Compton-scattered in the atmosphere, therefore arriving both later and at lower energy. Grefenstette et al. (2008) also noted that the TGFs were so bright that they were saturating the LADs, and showed that the result of this instrumental effect is to exaggerate both the magnitude and the delay time of the Compton tails, and to soften the summed spectrum (since most of the saturation occurs at the peak, when the spectrum is hardest). Gjesteland et al. (2010) verified this result and concluded that the BATSE TGFs are on average 3 to 4 times brighter than is apparent from the saturated LAD data.

4.2 Observations of Terrestrial Gamma-Ray Flashes: the RHESSI Era

\subsubsection{RHESSI Instrumental Details}

The second source of TGF data was the Reuven Ramaty High Energy Spectroscopic Imager (RHESSI) satellite, a NASA Small Explorer dedicated to studying solar flares with an array of nine high-resolution germanium detectors (Lin et al. 2002; Smith et al. 2002). RHESSI was launched in 2002 February into a $580 \mathrm{~km}, 38^{\circ}$-inclination orbit, and is still operating as 
of this writing. While the instrument is much smaller than BATSE, and consequently detects only a few tens of photons in each TGF, it has several advantages that make the RHESSI data complementary to BATSE. First, RHESSI returns to the ground data on every photon detected, without an onboard trigger, meaning that data can be searched with continuouslyrefined algorithms on the ground for TGF events (Grefenstette et al. 2009; Gjesteland et al. 2012). This produced a detection rate for RHESSI TGFs about an order of magnitude higher than the average rate during BATSE's lifetime; over 1000 TGFs have been found in the RHESSI data between 2002 and the present, with possibly three times as many, in total, being detectable in the data with an improved algorithm (Gjesteland et al. 2012). Second, RHESSI produces high-energy spectra of TGFs that run from $30 \mathrm{keV}$ to $17 \mathrm{MeV}$. While no one RHESSI TGF has enough counts to make a spectrum (there are typically from 17 to 40 counts in each), the sum of many RHESSI TGFs allows for detailed spectral fitting (Dwyer and Smith 2005; Carlson et al. 2007; Babich et al. 2008e; Hazelton et al. 2009; Hazelton 2009; Grefenstette et al. 2009). Finally, the higher orbital inclination ( $38^{\circ}$ versus $28^{\circ}$ ) allowed a search for TGFs in the temperate zones, showing that they are less common there than in the tropics, even relative to lightning (Smith et al. 2005, 2010; Williams et al. 2006). RHESSI was not built with GPS synchronization for absolute timing, and millisecond uncertainties in its timing have complicated the comparison of TGF timing with the timing of lightning throughout the mission (see Sect. 4.4 below). The bright outburst of a Soft Gamma Repeater (neutron star) in 2005 was used to connect RHESSI's timing solution to that of the Swift spacecraft, which has a trusted absolute time. This gave a $+1.8 \mathrm{~ms}$ correction to the RHESSI clock (Grefenstette et al. 2009).

\subsubsection{RHESSI Measurement Summary}

Smith et al. (2005) presented the first RHESSI results, including the extension of the spectrum up to $20 \mathrm{MeV}$ and the higher occurrence rate discovered by searching the data on the ground. Grefenstette et al. (2009) presented a more complete survey of the RHESSI data. They found that even the RHESSI detectors, which are much smaller than BATSE's, often still saturate during TGF peaks. The RHESSI data sample contained a much higher fraction of short, single-peaked TGFs than the BATSE sample. This was interpreted as a bias for longer-lasting or multi-peaked events in BATSE due to the nature of the onboard trigger: a short TGF of ordinary brightness would not produce enough counts to appear significant over $64 \mathrm{~ms}$ of BATSE's background. In contrast, the RHESSI search algorithm used a $1 \mathrm{~ms}$ window, more typical of actual TGF durations. Other conclusions from Grefenstette et al. (2009) included an upper limit on gamma-ray emission leading up to or following TGFs, and negative results for spectral differences based on brightness, latitude, or time of day. Collier et al. (2011) have noted that, as expected, TGFs detected at greater distances from RHESSI are less likely to produce a large number of counts in the instrument, and they have found some statistical support for a power-law distribution of intrinsic TGF intensities.

Spectral differences among TGFs were first noted by Nemiroff et al. (1997). Østgaard et al. (2008) noted that more distant TGFs (relative to the sub-satellite point) should be softer, and indeed found such a correlation, using the relative response of the BATSE detectors themselves to approximately calculate the sub-satellite distance. There are two physical effects that contribute to this softening with distance for upward-directed runaway electrons: the bremsstrahlung process itself produces a beam that is hardest in the forward direction, and Compton scattering produces a soft component that extends to large angles. Hazelton et al. (2009) found that RHESSI TGFs at large distances are softer, using an independent data set (sferics from the World Wide Lightning Location Network, or WWLLN) to find the position of the storm producing the TGF. 


\subsubsection{TGF Spectra and Source Altitude}

The production altitude of TGFs was initially a mystery that has since been solved through both detailed TGF spectral analysis and through measurements of the associated lightning (discussed in Sect. 4.4). The difficulty of altitude measurements from TGF spectra is primarily due to two factors: first, the limited spectral information available from the BATSE data, and second, an over-simplified view of the process by which the gamma-ray spectrum would be altered on its way out of the atmosphere. For example, Smith et al. (2005) used the photoelectric absorption cross-section in air to argue that the presence of photons below $\sim 60 \mathrm{keV}$ in both the BATSE and RHESSI spectra implied a high-altitude origin $(>25 \mathrm{~km})$. This argument neglected the possibility of the production of such photons at high altitudes via Compton-scattering of higher-energy, more penetrating gammas from below. Dwyer and Smith (2005), Carlson et al. (2007), Babich et al. (2008e) and Hazelton et al. (2009) simulated the full physics of gamma-ray propagation in the atmosphere to match the RHESSI spectrum of the sum of many TGFs and found that the relativistic runaway electron avalanche (RREA) model fits well if the source altitude is comparable to that of thunderstorms (below $\sim 20 \mathrm{~km}$ ). From the ensemble spectra of nearby and distant RHESSI TGFs, Hazelton et al. found that the model fitting the two data sets best was a significantly broadened beam at $15 \mathrm{~km}$ source altitude, in agreement with earlier simulations (Dwyer and Smith 2005; Carlson et al. 2007). Østgaard et al. (2008) and Gjesteland et al. (2010) did the same for the four-channel BATSE data, and found that, once the effects of deadtime in the BATSE detectors were accounted for (Grefenstette et al. 2008; Gjesteland et al. 2010), the same low source altitudes were most consistent with BATSE as well. Gjesteland et al. (2011) compared observation angles of RHESSI TGFs (with respect to lightning sferic locations) with Monte Carlo simulations and also found that for a low altitude source the data are most consistent with wide gamma-ray beams with half angles $>30$ degrees. Figure 21, from Dwyer and Smith (2005), shows the RHESSI summed TGF spectrum along with the relativistic runaway (REAM) model at several altitudes, demonstrating that even TGFs deep in the atmosphere produce significant flux $<100 \mathrm{keV}$, and that the best diagnostic of depth comes from the spectral slope in the range of approximately $0.5 \mathrm{MeV}$ to $4 \mathrm{MeV}$. A comparison of the RHESSI TGF spectrum and the ground-level event seen by Dwyer et al. (2004a) can be found in Dwyer (2009).

\subsubsection{RHESSI TGF Geographic Distribution and Meteorology}

The large number of RHESSI TGFs allows detailed comparisons of the geographical distribution of TGFs with other meteorological phenomena. Figure 22 shows the global distribution of 805 TGFs measured by RHESSI. Williams et al. (2006) compared the TGF distribution with the global occurrence of lightning and found deficits of TGFs at high latitude. They offered the explanation that if TGFs are associated with intracloud lightning, the altitude at which the TGFs occur may be higher in the tropics, where the tropopause and thunderstorm tops are higher. Because gamma-rays more easily escape into space at higher altitude, TGFs would be more frequently detected in the tropics. Smith et al. (2010) did a more detailed comparison of the TGF map with the map of lightning produced by NASA's Lightning Image Sensor and Optical Transient Detector instruments (Christian et al. 2003). They found that considering more complete Monte Carlo simulations of atmospheric propagation, the altitude effect was not as strong as originally expected, with the attenuation in the total number of gammas having an $e$-folding depth of $45 \mathrm{~g} \mathrm{~cm}^{-2}$ of overlying atmosphere. The prevalence of TGFs in the tropics may therefore have a more complicated explanation 


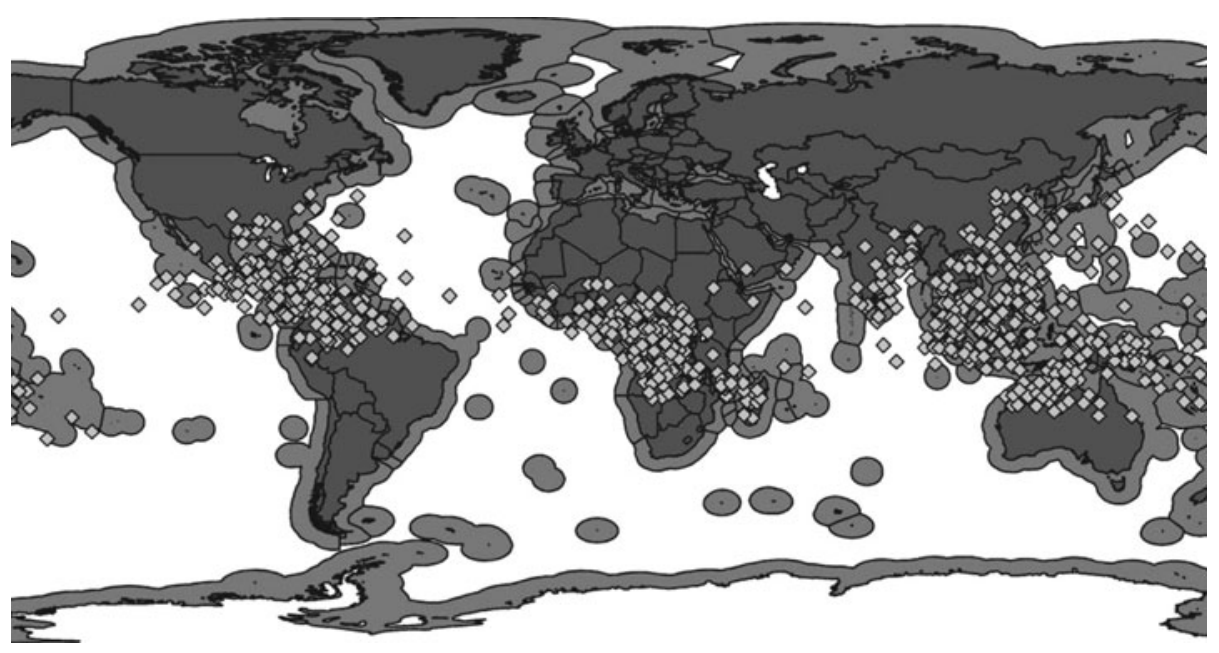

Fig. 22 Map of the locations of 805 RHESSI TGFs (diamonds). Coastal regions are shown in gray. From Splitt et al. (2010)

than simply gamma-ray escape. Smith et al. (2010) also found other anomalies, such as a lower TGF/lightning ratio in Africa relative to the Americas and the Maritime Continent and a shift in longitude between the peaks of lightning and TGFs within the Maritime Continent.

A significant part of the difference between lightning and TGF distributions may therefore lie in meteorological conditions that favor the production of TGFs, not just their escape into space. Splitt et al. (2010) made an extensive study of storms associated with individual RHESSI TGFs and compared the TGF map to maps of water vapor and ice content, showing that ice alone had a poor correlation with the TGF map but that liquid water at 10-14 km, indicating deep convection, gave a much better match. Elevated mixed phase (liquid water plus ice), which is expected to correlate with electrification, might be the most physically relevant parameter; in a case study in Splitt et al. (2010), one TGF-producing storm had several regions of elevated mixed phase. The authors found that storm systems of all sizes could produce TGFs, from storms with a cloud-top size of $<300 \mathrm{~km}^{2}$ to large mesoscale convective complexes (MCCs) of $10^{5} \mathrm{~km}^{2}$. These storms were on average tall $(13.6 \mathrm{~km}$ to $17.3 \mathrm{~km}$ ). Hazelton (2009) found that the TGF/lightning ratio was higher for coastal regions than for inland or oceanic regions. The meteorological pattern of TGF occurrence, while subtly different from that of lightning, is much more distinct from that of elves (expanding glows within the ionosphere caused by lightning), which are relatively much more common over the ocean than lightning in general, and sprites (discharges in the upper atmosphere caused by lightning), which are less likely than TGFs to avoid mid-latitudes (Chen et al. 2008).

The time of occurrence as well as the spatial distribution of TGFs may give clues as to their cause and mechanism. Splitt et al. (2010) found enhanced production of TGFs relative to lightning between 0300 and 0900 hours local time. Otherwise, the diurnal and seasonal cycle of TGFs is similar to that of lightning (Hazelton 2009; Splitt et al. 2010). Smith et al. (2010) used WWLLN data to place RHESSI TGFs in the context of the evolution of flash rate within the storm that produced them, finding that TGFs tend to come after the peak flash rate of the storm, with an average delay of $38 \mathrm{~min}$ but a standard deviation of $98 \mathrm{~min}$. 


\subsubsection{SONG-D}

From 2001-2005, the gamma-ray spectrometer SONG-D (a single large crystal of CsI) on the Russian solar physics satellite CORONAS-F observed several events with time profiles suggestive of TGFs (Arkhangelskaja 2006). One of these events was associated with tropical cylone Beni.

\subsection{Recent and Upcoming Observations of Terrestrial Gamma-Ray Flashes}

Two more missions, each with its own unique capabilities, have begun collecting TGF data in the past few years. Fermi, a NASA spacecraft, and AGILE (Astro rivelatore Gamma a Immagini LEggero), an Italian mission, are both gamma-ray telescopes for astrophysics in the $\mathrm{MeV}$ to $\mathrm{GeV}$ range. Airborne measurements from the ADELE instrument have also yielded a single TGF observation with interesting implications (Smith et al. 2011a).

\subsubsection{Fermi TGF Observations}

Fermi observes TGFs in its Gamma-ray Burst Monitor (GBM) instrument (Meegan et al. 2009). GBM consists of 12 uncollimated NaI scintillators pointing in different directions and two large bismuth germanate (BGO) scintillators, the former primarily for photons up to $1 \mathrm{MeV}$ and the latter operating up to $40 \mathrm{MeV}$. Like BATSE, GBM usually operates in a triggered mode, but with a $16 \mathrm{~ms}$ accumulation time, making it more easily able to trigger on TGFs than BATSE. Recently, GBM has been able to operate in a photon-by-photon mode similar to RHESSI's during parts of its orbit (Briggs 2011). Since its overall collecting area is much greater than RHESSI's, this represents the most sensitive data set ever taken for TGF detection.

GBM provides the best spectral information available to date for each individual TGF, with the combination of large effective area, good time and energy resolution, and large energy range. Briggs et al. (2010) presented the first results, including a $38 \mathrm{MeV}$ photon in one TGF and the fastest detected risetime $(7 \mu \mathrm{s})$, a result that requires a large number of counts per event. Further analysis of the time profiles of GBM TGFs was presented by Fishman et al. (2011), including more examples of fast rise and fall times and some events with durations as low as $50 \mu \mathrm{s}$, about a factor of 4 shorter than any of the RHESSI events. Note that the current RHESSI detection algorithms reject events $<100 \mu$ s (Grefenstette et al. 2009; Gjesteland et al. 2012). Spectral modeling of individual GBM TGFs where the distance to the originating lightning is known promises better constraints in the future for the beaming characteristics of TGFs (angular width and possible tilting of the beam).

\subsubsection{AGILE TGF Observations}

AGILE (Tavani et al. 2009), like the Large Area Telescope (LAT) on Fermi, is a tracking detector that determines the direction of incoming high-energy gammas by creating a shower in the instrument, and collects the remaining energy in a "mini-calorimeter" (MCAL) made of CsI crystals at the bottom (Labanti et al. 2009). AGILE primarily detects TGFs in the CsI. Marisaldi et al. (2010a) used these data to study the geographical and diurnal distributions and the spectrum of TGFs, finding that all were consistent with the RHESSI results, but since the MCAL has a broader energy range, they were able to detect individual photons up to $40 \mathrm{MeV}$. Fuschino et al. (2011) compared the TGF and LIS/OTD lightning geographical distributions. For the narrow equatorial band sampled by AGILE ( $2.5^{\circ}$ orbital inclination), 
Fig. 23 Summed spectrum of 130 AGILE TGFs from Tavani et al. (2011) showing the high-energy excess above the exponential decay typical of relativistic runaway emission (dashed line). Copyright 2011 by the American Physical Society

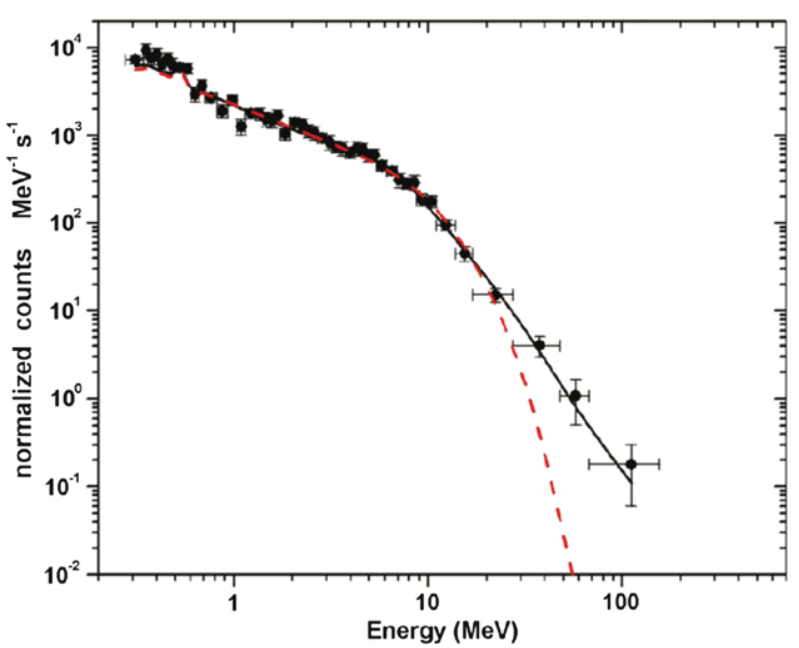

they found a much better agreement than Smith et al. (2010) did for the RHESSI TGFs over a broader range of latitudes. They estimated a global TGF/lightning ratio of $8 \times 10^{-5}$ for an estimated TGF rate of 220-570 TGFs per day (compared to the early estimate of at least 50 per day from Smith et al. 2005).

Tavani et al. (2011) summed MCAL spectra from 130 events to derive the spectrum shown in Fig. 23. The result, perhaps the most surprising in the study of TGFs over the last few years, was that there is a power-law component to the spectrum up to $100 \mathrm{MeV}$. While there is nothing in the accepted runaway mechanism that forbids making electrons (and bremsstrahlung) photons this energetic, the avalanche physics requires the continuous production of new electrons at lower energies, which means the spectrum must retain its exponential form. In other words, any $100 \mathrm{MeV}$ photons would have to be accompanied by a far greater flux of photons at lower energy than is seen. While there has yet to be a model proposed to explain the MCAL summed spectrum, this result has stimulated considerable interest in the community. Fermi is, as of this writing, just completing a series of observations in which the powerful Large Area Telescope is turned to face the Earth to measure TGFs (Eric Grove, private communication). The sensitivity of the LAT to gamma-rays on the order of $100 \mathrm{MeV}$ is greater than that of any other satellite, and special software is being used for these observations to extend this advantage down to $20 \mathrm{MeV}$. If the issues raised by the high instantaneous flux of TGFs can be worked out, these observations could provide a strong confirmation or rebuttal of the AGILE result.

The silicon tracker on AGILE has detected gamma-rays associated with TGFs that triggered MCAL (Marisaldi et al. 2010b). These 9 photons, from 8 individual TGFs, had an average energy of $60 \mathrm{MeV}$ and could be tracked with a typical angular precision of $5.8^{\circ}$, resulting in a range of positions for the 8 TGFs between $100 \mathrm{~km}$ and $390 \mathrm{~km}$ of the satellite footpoint. This range of distances is in agreement with the ranges that have been derived using sferic data (Cummer et al. 2005; Hazelton et al. 2009; Cohen et al. 2010b; Connaughton et al. 2010; Collier et al. 2011).

\subsubsection{ADELE TGF Observations}

Recently, an airborne detector made the first detection of a TGF from an altitude comparable to its production altitude. The Airborne Detector for Energetic Lightning Emissions 
(ADELE), flying at $14 \mathrm{~km}$ aboard the Gulfstream $\mathrm{V}$ jet operated by NOAA, saw a TGF at the same time as a +IC flash in an active cell $10 \mathrm{~km}$ away over the Georgia coast in the southeastern United States (Smith et al. 2011a). The calculated luminosity of this event given the $10 \mathrm{~km}$ distance was comparable to that associated with TGFs seen from space (about $10^{17}$ relativistic electrons needed). Perhaps more importantly, ADELE passed within $10 \mathrm{~km}$ of over 1000 other discharges detected by ground-based sferic networks without detecting any other TGF (Smith et al. 2011b). The authors concluded that TGFs, even if there was a population too faint to be seen from orbit, are rare events, only associated with $0.1-1 \%$ of lightning flashes. Even at that rate, however, the global rate would be several thousand per day.

\subsubsection{Upcoming Instrumentation}

Now, for the first time, instruments designed specifically to study TGFs and related phenomena from orbit are in flight or in preparation. The first such instrument to be launched was the gamma-ray spectrometer onboard the RISING microsatellite in January 2009 (Yoshida et al. 2010). Molinya-Gamma, a Russian experiment on the International Space Station (ISS), was installed in February 2011, with gamma-ray detectors covering the range $0.3-1 \mathrm{MeV}$ and 16 ms time resolution (Kuznetsov et al. 2011). Upcoming missions include the French microsatellite TARANIS (Lefeuvre et al. 2008), the European ASIM experiment for ISS (Neubert 2009), and the US nanosatellite Firefly (Rowland et al. 2009) and its sister instrument, called Firestation, for ISS. While varying significantly in scope, specialization, and capabilities, what all these missions have in common is additional instrumentation to simultaneously study optical emission from lightning and transient luminous events such as sprites along with high-energy radiation.

In addition to the challenge of clarifying the association of TGFs with lightning and with TLEs (if any), some of the primary future challenges will be identifying the distribution functions of TGFs in luminosity, altitude, and beaming angles (width and tilt). Simple distributions in these quantities were tested by Hazelton (2009). One difficulty lies in the ability of all these quantities at once to affect the spectral hardness and fluence of a given TGF in ways that are hard to disentangle. Furthermore, instrumental triggering thresholds and deadtime effects complicate the process of deriving the intrinsic luminosity distribution. Carlson et al. (2011) addressed these issues in depth (see also Collier et al. 2011). Fermi/GBM and future missions have a superior dynamic range between the detection threshold and saturation in comparison to BATSE and RHESSI, so the upcoming large TGF databases should provide better opportunities to constrain the intrinsic luminosity distribution. Further observations from aircraft and balloons will constrain the luminosity distribution at the faintest end.

\subsection{Insight from Radio Observations of TGF-Associated Lightning}

The paper that reported the discovery of TGFs (Fishman et al. 1994) used satellite imagery to establish their connection to storms. This connection to storms in general and, more specifically, to some form of lightning process has been confirmed by many studies since. But the precise relationship between TGFs and lightning, and what kind of lightning processes are involved, remains incompletely answered.

The first effort to address this issue (Inan et al. 1996) studied two BATSE-detected TGFs and found, from very low frequency (VLF) radio recordings, that lightning was occurring in storms under the satellite at the times of TGF detection. Moreover, in one of those cases a 
clear VLF-radiating lightning process occurred within $1.5 \mathrm{~ms}$ of the TGF. This process was a very strong radiator relative to other lightning in the same storm, indicating substantial charge motion in the radiating process.

The observation that the TGF-associated lightning process could be strong in a relative sense reinforced (incorrectly, as it turns out) the theoretical idea (Bell et al. 1995; Gurevich et al. 1996; Lehtinen et al. 1996; Roussel-Dupré and Gurevich 1996) that TGFs were produced at high altitudes (approximately 30 to $80 \mathrm{~km}$ ) by the same post-discharge electric fields that produce high altitude mesospheric breakdown in the form of sprites (Pasko 2007). This idea was aggressively pursued in the literature, as at the time there were no other measurements of TGF-associated lightning to constrain theory and modeling. It should be noted, though, that the notion that sprite optical emissions are produced by RREA was effectively disproved by observations that sprites are composed of filamentary streamers expected for conventional breakdown (Gerken et al. 2000), and at present there are no reported observations of high altitude optical emissions associated with the runaway avalanche process.

\subsubsection{RHESSI TGFs and Lightning}

The study of TGF-associated lightning processes was resurrected with new observations of TGFs from the RHESSI satellite (Smith et al. 2005). Prompt reporting of RHESSI TGFs enabled the careful coordination with ground-based radio observations of lightning, which led to a number of important findings that began to create a picture of some aspects of TGF-associated lightning processes.

The analysis of $30 \mathrm{kHz}$ and lower frequency radio emissions during 26 TGFs (Cummer et al. 2005) showed that 13 of them occurred within several milliseconds of detectable positive polarity (those that transfer positive charge downward) lightning processes. This strengthened the connection between TGFs and some form of fast lightning process, although the few-ms timing uncertainty in the RHESSI data (Grefenstette et al. 2009; see Sect. 4.2.1) prevented determining whether the lightning process comes before or after the TGF.

Quantitative analysis of these low frequency radio emissions, summarized in Fig. 24, also showed that the TGF-associated charge moment changes were 50-500 times too small

Fig. 24 Figure from Cummer et al. (2005) comparing the inferred altitude profile of electric field following two measured TGFs to that predicted by Lehtinen et al. (2001) to produce a TGF by high altitude RREA. The measurements are 50 times smaller than the predictions, confirming that observed TGFs are not produced by high altitude fields and, consequently, must be produced at low or thundercloud altitudes

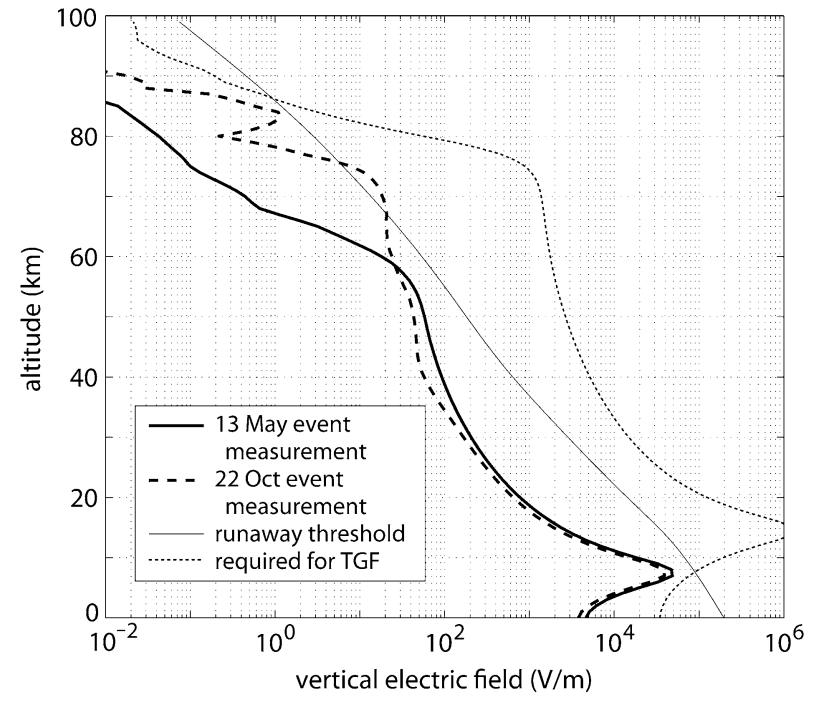


to drive the RREA process at high altitudes. Consequently, the only place where the electric field could be high enough was close to or within the thundercloud, and the prevailing theoretical mechanism of high altitude TGF production described in the literature is not the mechanism responsible for the observed RHESSI TGFs. An analysis based on direction finding and event timing also suggested that most of the TGF-associated lightning occurred within approximately $300 \mathrm{~km}$ of the satellite footprint.

An analysis of higher frequency radio measurements (Stanley et al. 2006), extending to several hundred $\mathrm{kHz}$, revealed more important details regarding TGF-associated lightning processes. Waveform details for 5 TGFs showed that the associated lightning was unambiguously positive polarity in-cloud ( $+\mathrm{IC}$, involving the upward motion of negative charge) and that the signals were radiated from a source altitude ranging from $11.5 \mathrm{~km}$ to $13.6 \mathrm{~km}$. Although the altitudes of the radiating lightning process and the gamma-ray production are not necessarily identical, this provided direct evidence that TGF production is an in-cloud process. These processes were produced by equivalent peak currents ranging from $15 \mathrm{kA}$ to $57 \mathrm{kA}$. This is very high for an $+\mathrm{IC}$ process, but is not nearly large enough for the electromagnetic pulse mechanism of TGF production (Inan and Lehtinen 2005) to operate.

A broader study of a larger number of RHESSI TGFs (Inan et al. 2006) showed that most are closely associated in time with detectable sferics with the same several-ms uncertainty driven by the uncertainty in RHESSI absolute timing. The quantitative characteristics of these sferics, namely relatively high peak current but generally modest charge moment change compared to other sferics in the same storm, demonstrated that the characteristics described above apply broadly to TGF-associated lightning. A re-examination of the BATSE events (Cohen et al. 2006) found more lightning-TGF temporal correlations that fit the same general picture. Intriguingly, but also confusingly, this work uncovered examples of multiple discrete TGFs over several milliseconds that in one case was associated with the same number of discrete sferics, but in one case was not.

With measurements of 36 RHESSI TGFs the spatial relationship between lightning location and TGF detection was also put on solid footing (Cohen et al. 2010b). Using multilocation VLF sferic measurements to geolocate the associated lightning, it was found that the majority of TGFs are detected within $300 \mathrm{~km}$ of the associated lightning process, as shown in Fig. 25, but that this distance extends to $700 \mathrm{~km}$ or more for a small fraction of TGFs. Again the TGF-lightning time relationship was confirmed to within a few milliseconds but could not be evaluated with more precision.

Most recently, an analysis of the broadband radio emissions from 56 RHESSI TGFs (Lu et al. 2011) showed that essentially all of them (96\%) were associated within several milliseconds of detectable VLF sferics. In roughly half of cases these sferics were isolated, single sferics, while in the other half multiple sferics were detected. The sferic multiplicity was not generally connected to the multiplicity of the TGFs. Regardless of the sferic multiplicity, there was always a single pulse observable at frequencies below $1 \mathrm{kHz}$ that reflects substantial charge motion on several millisecond time scales. Effective peak currents spanned a wide range from a few tens to a few hundred $\mathrm{kA}$, and total charge moment change spanned a similarly wide range from a few tens to a few hundred $\mathrm{C} \mathrm{km}$.

\subsubsection{Lightning Flash Structure and TGFs}

The observations described above showed conclusively that TGFs were associated with IC lightning processes. However, IC lightning flashes commonly have durations of several hundred milliseconds during which many different processes occur (Shao and Krehbiel 1996). Determining when and where in an IC lightning flash TGFs occur would provide important constrains on TGF source mechanisms. The first substantial effort towards this goal 

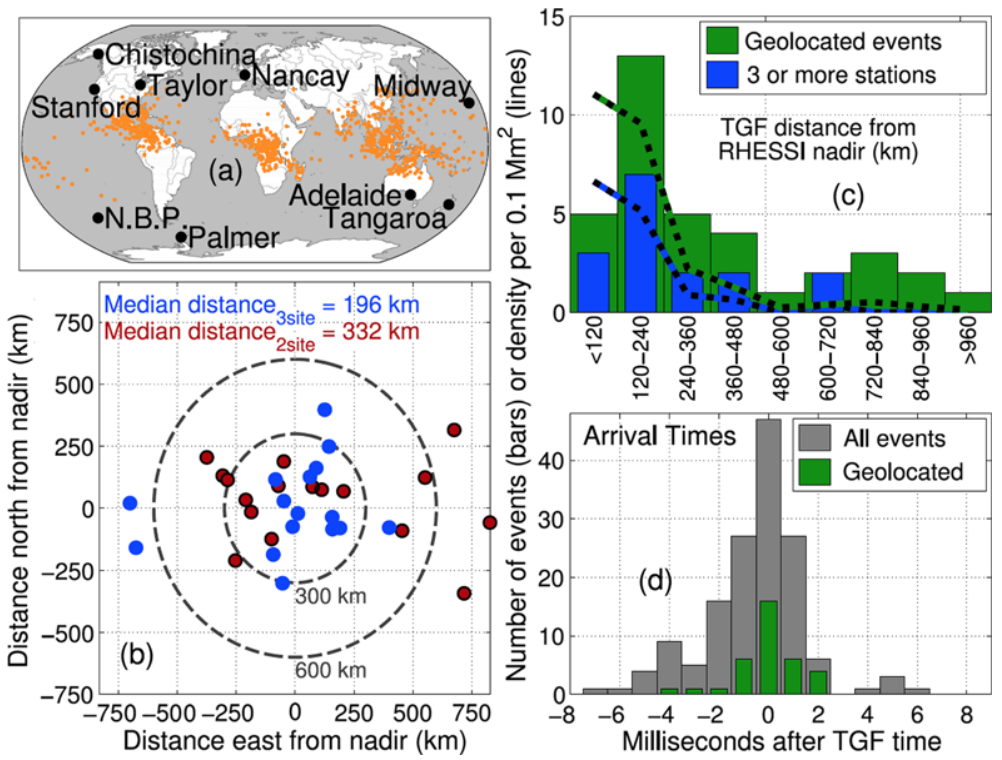

Fig. 25 Figure from Cohen et al. (2010b) summarizing the location and timing of lightning signals associated with 36 RHESSI-detected TGFs. The TGFs all occurred within several ms of the lightning process, in agreement with previous measurements. Importantly, these measurements defined the spatial relationship of the RHESSI satellite and the source lightning, showing that most TGFs are generated within $300 \mathrm{~km}$ of the RHESSI nadir point

analyzed multi-station radio measurements for 9 RHESSI events (Shao et al. 2010). It was again found that all 9 events were associated with IC processes. The source altitudes of radio pulses within several ms of the TGF were 10.5 to $14.1 \mathrm{~km}$, in agreement with prior observations of fewer events (Stanley et al. 2006). In a subset of events that had low noise measurements, it was found that these TGFs were produced during a sequence of LF pulses at steadily increasing altitudes that were consistent with upward IC leader development that occurs in the first 5 to $10 \mathrm{~ms}$ of an IC flash.

This basic picture was conclusively demonstrated ( $\mathrm{Lu}$ et al. 2010) with detailed measurements of a single RHESSI TGF that was fortuitously located close enough to a VHF lightning mapping array (LMA) (Rison et al. 1999) to obtain detailed measurements of the flash and discharge structure at the time of the TGF, as shown in Fig. 26. The TGF occurred $3 \mathrm{~ms}$ after the onset of the flash (even with the RHESSI timing uncertainty this was definitively after the flash onset) when the upward negative leader tip had reached an altitude between 10 and $11 \mathrm{~km}$. The main negative and positive charge layers in the originating region of the storm were centered at 8.5 and $13.0 \mathrm{~km}$, respectively, constraining the region of highest electric field to between these altitudes. This particular IC lightning flash initiated with a high VHF power radio emission characteristic of so-called narrow bipolar events (NBE) or compact intra-cloud discharges (CIDs) (Le Vine 1980; Smith et al. 1999).

During the period of upward leader propagation, the radio emissions from this event encompassed a wide range of temporal scales. A sequence of at least 5 distinct fast pulses (approximately 10-20 $\mu$ s time scale) occurred within roughly $1 \mathrm{~ms}$ of the TGF. These pulses were superimposed on a much slower, several ms radio pulse produced by a total charge moment change $(90 \mathrm{C} \mathrm{km}$ ) on this time scale that was unusually large for the early stages of 


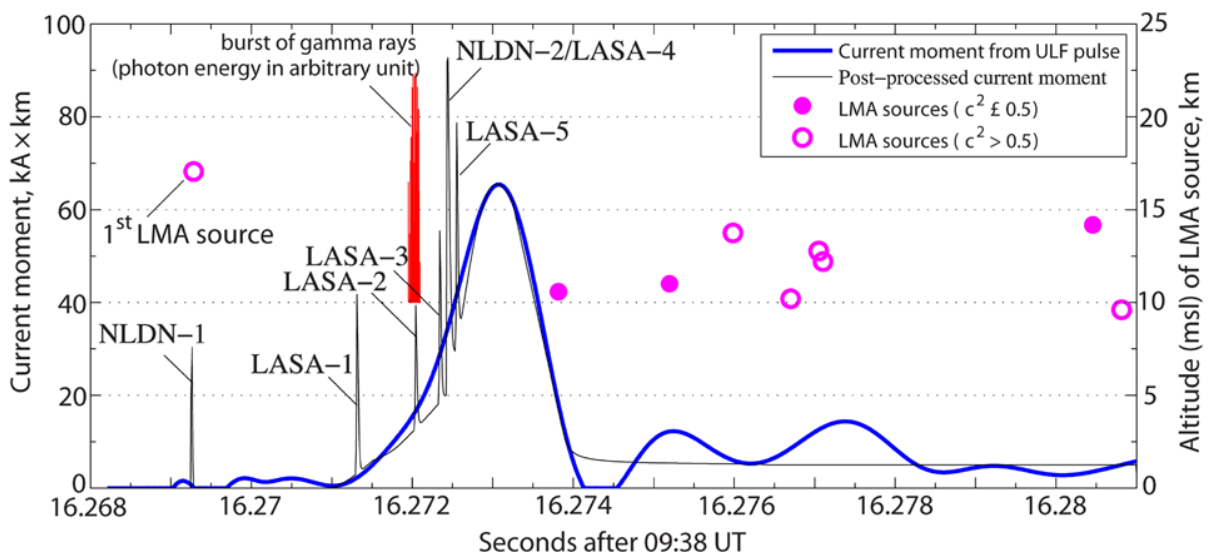

Fig. 26 Figure from Lu et al. (2010) showing the details for an TGF measured with a VHF lightning mapping array. The TGF occurred within $3 \mathrm{~ms}$ of, but distinctly after (even subject to the RHESSI timing uncertainty) the onset of a normal in-cloud lightning flash. The gamma rays were generated while the initial negative polarity leader traveled upward and also during a sequence of short radio pulses superposed on a slower, ms-scale net charge transfer

an IC lightning flash. This combination of fast and slow processes is also consistent with the radio measurements of many RHESSI TGFs described above (Lu et al. 2011), and indicates that the detailed picture of IC lightning flash development obtained from this one events applies to at least most and perhaps all of the RHESSI TGFs.

\subsubsection{Fermi-GBM TGFs and Lightning}

With the launch of the GBM instrument on the Fermi satellite (described above in Sect. 4.3.1), the precise temporal relationship between lightning and TGFs could be determined because of the several microsecond absolute timing accuracy of the GBM measurements (Briggs et al. 2010). The first effort to correlate precise TGF and lightning times (Connaughton et al. 2010) compared lightning times reported by the World-Wide Lightning Location Network (WWLLN) (Rodger et al. 2009) and the times of peak TGF brightness. Out of 50 TGFs, 15 were found to be associated with WWLLN lightning detections. Of these 15,13 were found to have lightning and TGF peak times within a $40 \mu$ s window. This firmly established the near simultaneity of TGFs and a process that radiates strongly and impulsively at the VLF frequencies used by WWLLN.

Further understanding was provided through a detailed analysis of measurements from 2 GBM TGFs (Cummer et al. 2011). These events had continuous (not triggered) $1-400 \mathrm{kHz}$ radio measurements at relatively short range to the lightning and TGF $(\sim 500 \mathrm{~km})$ combined with high precision $(<2 \mathrm{~km}$ ) National Lightning Detection Network (NLDN) (Cummins et al. 2009) geolocations that enabled a timing comparison with approximately $15 \mu$ s absolute uncertainty in each case. The largest source of timing uncertainty is the uncertain gamma ray source altitude, which was assumed to be $13 \pm 3 \mathrm{~km}$. Around the time of the TGF, both of these events exhibited a sequence of fast processes (a few tens of $\mu$ s duration) completely consistent with past observations (Shao et al. 2010; Lu et al. 2010). These fast processes, possibly connected to leader stepping, did not occur with a consistent time relationship to the TGF. However, in both events the signal from a significantly slower process of 100-200 $\mu$ s duration was found to mirror the rise, peak, and fall of the gamma ray production almost 
perfectly (within $\sim 10 \mu \mathrm{s}$ ). This suggests that a specific radio signature of TGF production has been identified, although more events are needed to be sure. It is also distinctly possible that this is the radio signature of the TGF-generating process itself.

\subsection{Terrestrial Electron Beams (TEBs)}

When it was still thought that TGFs originated from high altitudes and were associated with sprites, it was logical to assume that some of the runaway electrons would also escape directly to space. This scenario was modeled by Lehtinen et al. (1997, 2000, 2001). Later, when it was shown that TGFs originate below about $20 \mathrm{~km}$, these electron beam models became moot, since the runaway electrons cannot escape to space from altitudes below $30 \mathrm{~km}$. Shortly after the low altitude range of TGFs was established, Dwyer et al. (2008b) showed that several BATSE events, previously identified as terrestrial gamma-ray flashes, were in fact high energy electron beams, which we suggest be referred to as Terrestrial Electron Beams (TEBs). Dwyer et al. (2008b) also proposed that the electron beams were secondary electrons and positrons generated by Compton scattering and pair production from the TGF gamma-rays in the upper atmosphere. At about $40 \mathrm{~km}$ altitude, there is still enough atmosphere for the TGF gamma-rays to interact with air, producing secondary electrons and positrons, but not so much atmosphere that the secondary particles are absorbed before they can escape to space. The TEBs, which follow the geomagnetic field line in the inner magnetosphere, may then be observed thousands of kilometers away by spacecraft. The REAM Monte Carlo simulations showed that the secondary electrons and positrons are confined to a beam with a radius of about $10 \mathrm{~km}$ (Dwyer et al. 2008b). Although the number of electrons and positrons that escape to space is only about $2 \%$ the number of gamma-rays, the electrons and positrons stay confined to the geomagnetic field line, so the fluence [particles $/ \mathrm{m}^{2}$ ] of the electrons and positrons does not change very much as they propagate away from the atmosphere. In contrast, the gamma-ray fluence falls off as $1 / r^{2}$, so that at spacecraft altitudes, e.g., $600 \mathrm{~km}$, the fluence of TEB electrons and positrons is actually larger than the fluence of gamma-rays from the TGF. On the other hand, because the TEB beam size is much smaller than the lateral extent of the TGF, it is expected that a spacecraft will find itself in a TEB about $1 \%$ as often as a TGF. However, triggered instruments such as CGRO/BATSE and Fermi/GBM probably preferentially trigger on the TEBs, since they have more particles and they are longer (from velocity dispersion) so dead time effects are less. Indeed about $15 \%$ of the BATSE TGFs appear to be TEBs and the very first TGF measured by Fermi/GBM turned out to be a TEB. In contrast, RHESSI saw only one clear electron-beam event in its first $1000+$ TGFs. While this is partially due to a greater efficiency of detecting short, ordinary TGFs, it is also a function of RHESSI's detector configuration: since the detectors are surrounded by several millimeters of aluminum, the sensitivity to electrons relative to photons is poorer than for CGRO/BATSE and Fermi/GBM.

As the TEB particles propagate away from the source region, they may be detected by spacecraft in low earth orbit on the same geomagnetic field line, either near the source region or near the geomagnetic conjugate point. Because the particles have a range of pitch angles relative to the magnetic field, they will also have a range of velocity components along the field line direction. This will result is a spreading of arrival times at the spacecraft due to velocity dispersion. Specifically, while most TGFs have durations less than few hundred microseconds, TEB are expected to have durations up to tens of milliseconds. In addition, if the spacecraft measures the TEB near the magnetic conjugate point and the magnetic field at the conjugate point is stronger than the field at the TEB source region, then some of the particles will be magnetically mirrored in the field below the spacecraft. These magnetically 

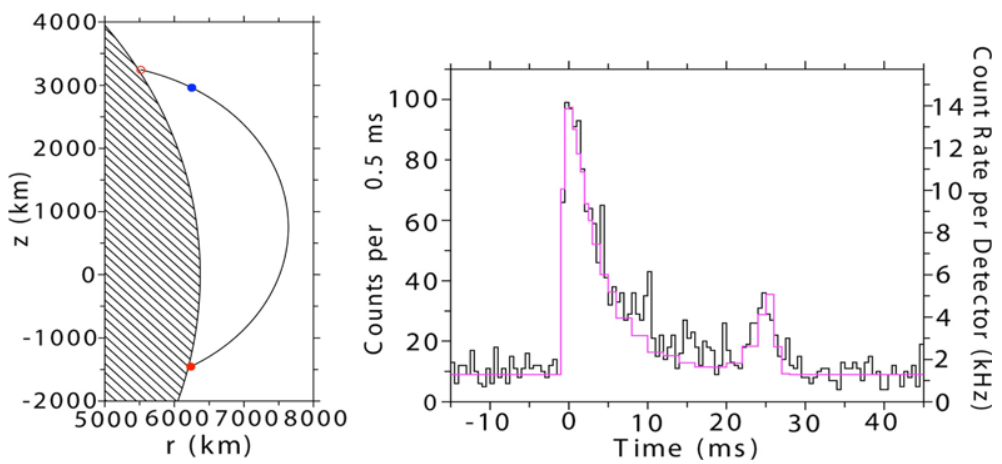

Fig. 27 Left: the geomagnetic field geometry for a terrestrial electron beam (TEB) event observed by the Fermi spacecraft. The shaded region represents the Earth and the solid line is the magnetic field line. The red dot shows the location of the TGF that created the TEB. The blue dot shows the location of Fermi. Right: Time intensity profile of the observed TEB (black). The red curve is the model prediction, including the magnetically mirrored component seen as the second pulse. Courtesy Briggs et al. (2011)

mirrored particles will also strike the spacecraft as they propagate back up the field line, resulting in a second pulse of particles. Because the arrival of this second pulse with respect to the first TEB pulse is determined by the geometry of the geomagnetic field line that the spacecraft is on, the separation between the two pulses may be determined and compared with observations. For one TEB event observed by BATSE over the Sahara desert, a clear signature of magnetically mirroring of the electrons in the geomagnetic field with the correct pulse separation was identified, demonstrating that the particles were indeed electrons and not gamma-rays (Dwyer et al. 2008b). Similar TEB events with second magnetic mirroring peaks were also seen by RHESSI and by Fermi/GBM (Briggs et al. 2011). Furthermore, Fermi/GBM also detected a very strong $511 \mathrm{keV}$ positron annihilation line in several events, providing solid evidence that it was being struck by electron-positron beams. Figure 27 shows one such electron-positron beam over the Sahara desert. The TGF that created the beam was located in a thunderstorm in south central Africa at the magnetic conjugate point that connected to the Fermi spacecraft. Along with the count rate (black) a model of the TEB that included the particle propagation through the inner magnetosphere is shown in red. Later, Cohen et al. (2010a) were able to identify the parent lightning flash associated with the electron beam at the magnetic foot point. The charge moment change of this lightning was too small to produce high altitude runaway electron avalanches, providing further support for the Dwyer et al. (2008b) model. Carlson et al. (2009b) conducted a search for TEBs using data from the SAMPEX satellite and found two candidate events with multiple strong pulses of electrons. Carlson et al. (2011) also performed a detailed study of TEBs, and, in particular, calculated the fluence and geometry of these events, inferring the relative detection probabilities of TGFs and TEBs.

An open question is what contribution the TEB electrons and positrons make to magnetosphere particle populations. The TEB particles are very energetic, with energies extending up to many tens of $\mathrm{MeV}$. Because they are created in the atmosphere, most will be reabsorbed in the atmosphere, either at the conjugate point or back at the source region after one bounce. In order to become trapped in the inner magnetosphere, the particles would have to experience pitch angle scattering during this trip. In particular, effects relating to the beam itself would have to cause the scattering (Lehtinen et al. 2000), since if pitch-angle scattering were common at these energies in the inner belt, the particles would be precipitated 
by further scattering shortly after becoming temporarily trapped. It is not clear at this time whether such scattering occurs.

\subsection{TGF Modeling}

Because the energy spectrum of the runaway electrons produced by RREA is mostly independent of the details of the source region, e.g. electric field configuration, density and seed population, the resulting gamma-ray spectrum at the source will also be insensitive to the details of the source model, that is, independent of how the seed particles are generated. As a result, computer simulations of the gamma-ray propagation through the atmosphere can be used to determine properties of the runaway electrons at the source, independent of the model. For example, Monte Carlo simulations have shown that the TGF source region has an altitude less than about $20 \mathrm{~km}$ and the number of runaway electrons at the source region is about $10^{17}$. It has been found that deeper sources fit the RHESSI data better when broad (45 degree) emission angles were used, perhaps indicating that the electric field lines at the source are either converging or diverging. The RREA spectrum has been found to fit the RHESSI data very well, up to its maximum energy of $17 \mathrm{MeV}$, lending support for the hypothesis that bremsstrahlung from RREAs is responsible for the emissions.

Specific TGF models can broadly be divided into the following three categories: upper atmospheric discharges (UADs), i.e., high altitude runaway electron avalanches acting on cosmic-rays, either at sprite altitudes (e.g., Roussel-Dupré and Gurevich 1996; Babich et al. 2001b, 2008b, 2011b; Inan 2005) or immediately above the thunderclouds (e.g., Babich et al. 2004c, 2007d, 2008c, 2008d, 2009a); lightning emission models (e.g., Moss et al. 2006; Dwyer 2008; Dwyer et al. 2010; Carlson et al. 2009a, 2010a); and relativistic feedback models (e.g., Dwyer 2003, 2008, 2012).

\subsubsection{Unsupported and Refuted Models}

In addition, several models exist that apparently are no longer being pursued. Paiva (2009) and Paiva et al. (2009) proposed non-standard mechanisms for TGFs, suggesting that TGFs are created by cosmic-ray muons stopped by very large electric fields in the atmosphere and that TGFs are created by the interaction of lightning-generated neutrons with nitrogen nuclei in air. However, both of these mechanisms are non-physical and disagree with TGF observations (Dwyer and Smith 2010; Smith 2011). In response to the comment by Dwyer and Smith (2010), Paiva et al. (2011) invoked an unphysically large average dielectric constant, i.e., $\sim 10$, for the thundercloud volume in order to address the criticism of using an unphysically large electric field in their model (i.e., twice the conventional breakdown field extending over $7 \mathrm{~km}$ ). Milikh and Valdivia (1999) modeled TGFs using a horizontal fractal lightning discharge that produced an electromagnetic pulse resulting in a stochastic runaway electron discharge in the stratosphere. Milikh et al. (2005) modeled TGFs as the result of a cosmic-ray air shower initiating a runaway electron beam during a thunderstorm. The runaway electron beam was subsequently propagated to higher altitudes by the interaction with whistler waves, propagating upwards in ducts produced by the self-focusing of these waves (Kaw et al. 2001). Given the initial flux of runaway electron assumed by the model, it is not clear if the propagation to higher altitudes is necessary or even consistent with TGF observations. Inan and Lehtinen (2005) proposed an EMP model of TGFs in which a very large cloud-to-ground return stroke produces an electromagnetic pulse that acts upon an extensive air shower in the upper atmosphere. Lightning observations of TGFs, which show that most TGFs are associated with intra-cloud lightning (see Sect. 4.4.2) of relatively modest peak 
current (see Sect. 4.4.1), cast doubt on this mechanism. Finally, Fargion (2002) suggested that upward going air showers produced by ultra-high-energy neutrinos may be responsible for TGFs. This model has difficulty explaining the multiple-pulse TGFs and the known association with thunderstorms and lightning. The association of TGFs with air showers is also problematic according to Dwyer (2008) (see Sect. 4.6.2 below).

Gurevich et al. (2007) introduced a high-field model of runaway electron production and stated that the model could explain the TGF spectrum. The gamma-ray spectrum presented in the paper was simply $1 / \varepsilon_{p}$, with no further information about a high energy cut-off or the energy scale of the runaway electrons. Because this is just the bremsstrahlung emission spectrum, little information is given about the predicted energy spectrum from this model. This model was later compared with the gamma-ray spectrum measured at Tien Shan (Chubenko et al. 2009; also see Gurevich and Milikh 1999) and the balloon measurements made by Eack et al. (1996a). All three energy spectra were presented in integral form, since discriminator levels were used to determine the energies at Tien Shan. Even after converting the Eack et al. spectrum to integral form, the Eack et al. spectrum presented by Gurevich and Milikh (1999) differs substantially from the Eack et al. spectrum presented by Chubenko et al. (2009), with no explanation given. The three spectra presented by Chubenko et al. appear to be in close agreement. However, this is misleading since Eack et al. did not record data above $120 \mathrm{keV}$. As a result, the integral spectrum will be artificially steepened. Chubenko et al. (2009) interpreted the agreement between their ground based measurements, the thundercloud measurements by Eack et al. and their theoretical curves as evidence of runaway breakdown. Moreover, because the Eack et al. measurements also agreed with the earlier low-field runaway breakdown model (Gurevich and Milikh 1999), they stated that runaway breakdown produces the same energy spectrum over a very wide electric field range, spanning thundercloud fields to high fields near leader tips. In fact, it is not clear that any of these spectra are consistent with the RREA spectrum. For example, going from $40 \mathrm{keV}$ to $120 \mathrm{keV}$, the measured Chubenko et al. spectrum drops at least a factor of 10 and their theoretical curve drops at least a factor of 50. Meanwhile, using a similar configuration as the previous work (Gurevich and Milikh 1999; Gurevich et al. 2001b), REAM Monte Carlo calculations shows that the RREA spectrum is only reduced by a factor of 1.7 over these energies. Again, it is possible that large scattering angles could soften the RREA spectrum, but such a case, which was not discussed, would make it difficult to make definitive statements about the nature of the source mechanism.

As discussed below in Sect. 5.3, the sprite models have been ruled out, both by the small current moment changes of the lightning associated with the TGFs (Cummer et al. 2005) as well as the TGF source altitudes (Dwyer and Smith 2005; Carlson et al. 2007). These models generally assume that a large lightning discharge, with a large charge moment change, generates an electric field above the thundercloud that exceeds the RREA threshold, resulting in an upward propagating RREA. Recent sferics measurements also appear to eliminate TGFs produced immediately above the thunderclouds, since the TGFs often occur near the beginning of a + IC lightning flash before significant charge moments changes occur (see Sect. 4.4). These observations also rule out the EMP models, which require very large CG return strokes (i.e., peak currents $>450-700 \mathrm{kA}$ ).

\subsubsection{TGF Seed Particle Source}

Using existing measurements of the intensity and duration of BATSE and RHESSI TGFs, along with limits on the electric field set by the relativistic feedback mechanism, involving backward propagating positrons and x-rays, Dwyer (2008) found that TGFs cannot be produced by relativistic runaway electron avalanches (RREAs) acting on natural background 
radiation or extensive cosmic-ray air showers alone, as has been assumed by many previous models. For instance, relativistic feedback limits the RREA multiplication factor to below about $10^{5}$ for reasonable thundercloud electric fields (see Fig. 8). At thundercloud altitudes, the flux of seed particles from the ambient atmospheric cosmic-rays flux is about $10^{4} \mathrm{~m}^{2} \mathrm{~s}^{-1}$ (Hillas 1972). Therefore, in order to produce the $10^{17}$ runaway electrons in $200 \mu$ s known from TGF observations (Dwyer and Smith 2005), the RREA mechanism would require a lateral diameter of the TGF source region to be at least $800 \mathrm{~km}$, much too large for realistic thunderstorms, especially those that are known to be associated with TGFs (Splitt et al. 2010). It is possible that a rapid electric field increase above a thundercloud, caused by lightning charge transfer, could temporarily circumvent the relativistic feedback threshold, but, as discussed in Sect. 4.4, recent sferics measurements appear to also eliminate this possibility. Similarly, Dwyer (2008) also showed that extensive air showers seeding RREAs do not produce enough energetic runaway elections. Furthermore, the TGF pulse durations, the existence of multi-pulsed TGFs, and the rate of air showers do not agree with TGFs generated by extensive air showers.

Instead, Dwyer (2008) argued that there exist two viable mechanisms for generating TGFs: (1) energetic seed particle production involving the relativistic feedback mechanism, or (2) runaway electrons produced in the strong electric fields associated with lightning leaders or streamers, similar to the energetic radiation observed on the ground from lightning.

For mechanism 1, Dwyer (2008) showed that relativistic feedback naturally explains the intensity and time scale of TGFs. A plausible scenario is that either upward + IC lightning or thundercloud electrification (see Dwyer 2005b) drive the high field region inside the thundercloud above the relativistic feedback threshold. A rapid increase in the number of runaway electrons follows, according to Eq. (2.6) (for $\gamma>1$ ). The increase in conductivity resulting from the ionization caused by the runaway electrons then partially discharges the electric field causing the feedback factor to drop below 1 again. Calculations show that the number of runaway electrons generated and the duration of the event is consistent with TGFs (Dwyer 2008, 2012).

For mechanism 2, Dwyer et al. (2010) used the runaway electron luminosities measured by Saleh et al. (2009) to show that such a lightning leader propagating through a thundercloud field with RREA avalanche multiplication consistent with the relativistic feedback limits could indeed generate enough gamma-rays to make a TGF. Carlson et al. (2009a, 2010a) also did calculations of the runaway electron production from a lightning leader inside a thundercloud in order to explain TGFs. The work by Carlson et al. differed from the work by Dwyer (2008) and Dwyer et al. (2010) in that they assumed that the RREA multiplication occurred in the electric field generated mainly by the lightning channel, while Dwyer and Dwyer et al. assumed that the avalanche multiplication occurred mostly in the ambient thundercloud electric field.

\section{Discussion: What Is Known, What Is Not Known, and Open Questions}

As discussed in this review, many forms of energetic radiation have been measured in association with thunderclouds and lightning. Energetic radiation has been observed from space in the form of bright terrestrial gamma-ray flashes (TGFs). Secondary energetic electrons and positrons, created by TGFs in the upper atmosphere, have also been observed by spacecraft, sometimes thousands of kilometers away for the source thunderstorm. Long lasting gamma-ray glows from thunderclouds have been observed in situ by aircraft and balloons and on the ground. X-rays have been observed from natural and triggered cloud-to-ground 
lightning, during the dart, dart-stepped and stepped leader phases. Similar x-rays have also been observed during long laboratory sparks. Neutrons, possibly generated by these gammaray emissions, have also been observed. In contrast, to date, no evidence has been presented that sprites or other high altitude transient luminous events (TLEs) emit energetic radiation.

Although a great deal of literature exists that uses the relativistic runaway electron avalanche (RREA) mechanism, usually acting on cosmic-ray seed particles, to explain these various observations of energetic radiation in our atmosphere, in many cases it is not clear whether or not such models are correct. For the x-rays from lightning and laboratory sparks, RREA does not appear to apply. Similarly, relativistic feedback does not appear to apply to these x-ray emissions. Gurevich et al. (2007) attempted to reformulate the RREA mechanism for high electric fields in order to explain lightning leader emissions. However, for reasons discussed in Sects. 2.2 and 4.6, it is not clear if this idea is correct. Most researchers are currently modeling the x-ray emissions from lightning and laboratory sparks using the thermal runaway augmented by the Wilson runaway mechanism. It is conceivable that some thundercloud glows are also the result of such a process produced by lightning and/or smaller discharges within the thundercloud, although recent observations of energy spectra and flux of the gamma-rays glows supports the RREA mechanism seeded by atmospheric comicsrays. Relativistic feedback could be contributing to the flux of these glows, but it has not been established how often, if ever, feedback is important. Terrestrial gamma-ray flashes, on the other hand, appear to be a good candidate for the relativistic feedback mechanism, since it naturally predicts the correct fluences and time-scales for the emissions. Thermal runaway from in-cloud lightning leaders augmented by RREA, either in the electric field from the lightning or in the large-scale thundercloud field, is a viable alternative mechanism for TGFs. RREA acting on ambient cosmic-rays or extensive air showers does not appear to apply to most TGFs (see Sect. 4.6.2). The recent AGILE observations of $100 \mathrm{MeV}$ gamma-rays presents the first serious challenge to the RREA mechanism for TGFs.

As will be discussed in the next section, there exist other atmospheric phenomena that have been modeled using the RREA mechanism, including lightning initiation and narrow bipolar events (NBEs). However, many of these models have not taken into account the limits on the electric field and avalanche multiplication factors imposed by the relativistic feedback mechanism. As a result, theoreticians have sometimes "cranked up the high-voltage knob" without regard to whether such electric fields are even possible. The reader is advised to be cautious about calculations that invoke RREA without addressing the limits on the avalanche multiplication factor set by relativistic feedback.

\subsection{Runaway Electrons and Lightning Initiation}

\subsubsection{Possible Mechanisms}

How lightning is initiated inside thunderclouds is one of the great unsolved problems in the atmospheric sciences (Rakov and Uman 2003). From the conventional point of view, in order to form a lightning leader, the electric field at some place in the thundercloud must reach a large enough value for conventional breakdown to occur. In dry air at sea-level the conventional breakdown threshold, $E_{k}$, is about $3 \times 10^{6} \mathrm{~V} / \mathrm{m}$ (Raether 1964). When precipitation is present, this threshold is reduced to $1.0-1.4 \times 10^{6} \mathrm{~V} / \mathrm{m}$, depending upon the size and shape of the precipitants (Solomon et al. 2001). Decades of in situ electric field measurements have failed to find electric field strengths anywhere near the conventional breakdown threshold, even when the effects of precipitation are included (MacGorman and Rust 1998). Indeed, the maximum electric field strength, scaled to the equivalent field at 
sea level, appears to rarely exceed about $4.0 \times 10^{5} \mathrm{~V} / \mathrm{m}$, one third the required value with precipitation. However, this value is larger than the RREA threshold field. Indeed, in situ electric field measurements show that the electric field near the lightning initiation point sometimes exceeds the RREA threshold (Marshall et al. 2005)

It has been suggested that runaway electron avalanches seeded by cosmic-ray extensive air showers (EASs) could result in enough ionization to initiate lightning (Gurevich et al. 1999, 2003; Solomon et al. 2001, 2002; Petersen et al. 2008). This hypothesis has gained a great deal of attention in recent years, both from the scientific community and from the popular press (Dwyer 2005a; Gurevich and Zybin 2005). Unfortunately, so far, there is little observational evidence and only limited theoretical work to support this idea. Babich et al. (2009b) investigated the EAS-RREA mechanism, using a 2-dimensional numerical model of conducting channel evolution. They found that lightning discharges cannot be initiated by a joint action of EASs and RREAs. Dwyer (2005b, 2010) used Monte Carlo simulation to calculate the maximum conductivity produced by RREA seeded by large extensive air showers and found that lateral diffusion and the relativistic feedback limit on the amount of avalanche multiplication prevent the conductivity from reaching large enough values to initiate lightning according to the criteria for lightning initiation calculated by Gurevich et al. (1999) and Solomon et al. (2001). This work was further supported by conductivity calculations by Dwyer and Babich (2011). Although it cannot be ruled out that lightning initiation is sometimes caused by cosmic-ray extensive air showers, exactly how this might occur remains to be established.

On the other hand, it is possible that RREA, either with or without significant relativistic feedback, acting on the ambient cosmic-ray flux could discharge the large scale thunderstorm field in such a way that local electric field enhancements occur, potentially providing a high enough field region to allow lightning to initiate, possibly with the help of hydrometeors (Dwyer 2005b; Babich et al. 2011a). Figure 28 shows how RREAs can discharge the large scale field producing localized field enhancements (also see Petersen et al. 2008). Dwyer (2005b) also showed that such discharges can push the field above the relativistic feedback threshold, providing a mechanism for generating TGFs.

\subsubsection{Runaway Electron Discharge Mechanism for Thunderclouds}

Figure 28 illustrates how runaway electron avalanches can drastically modify the thundercloud electric field. As the thundercloud charges and the electric field increases, the discharge currents increase dramatically as the field approaches the relativistic feedback threshold. It is possible that a steady state configuration may be reached in which the charging currents are balanced by the discharge currents resulting from the runaway electron production. The gamma-ray glows that have been reported (see Sect. 3.2) may be a manifestation of this steady state configuration. In this case, runaway electron physics may be playing an important role in the electric fields of thunderclouds, which may affect lightning initiation. At the very least, runaway electrons probably play a significant role in discharging thunderclouds and need to be considered when modeling thundercloud electrification.

\subsubsection{The TGF-Lightning Connection}

As discussed in Sect. 5.1.1, how lightning is initiated and what conditions are present during the initiation process have remained a great puzzle. TGFs have been shown to occur at the beginning of intra-cloud lightning flashes, just after the initiation occurs. For instance, Behnke et al. (2005) measured the initial leader velocities during intracloud lightning and 


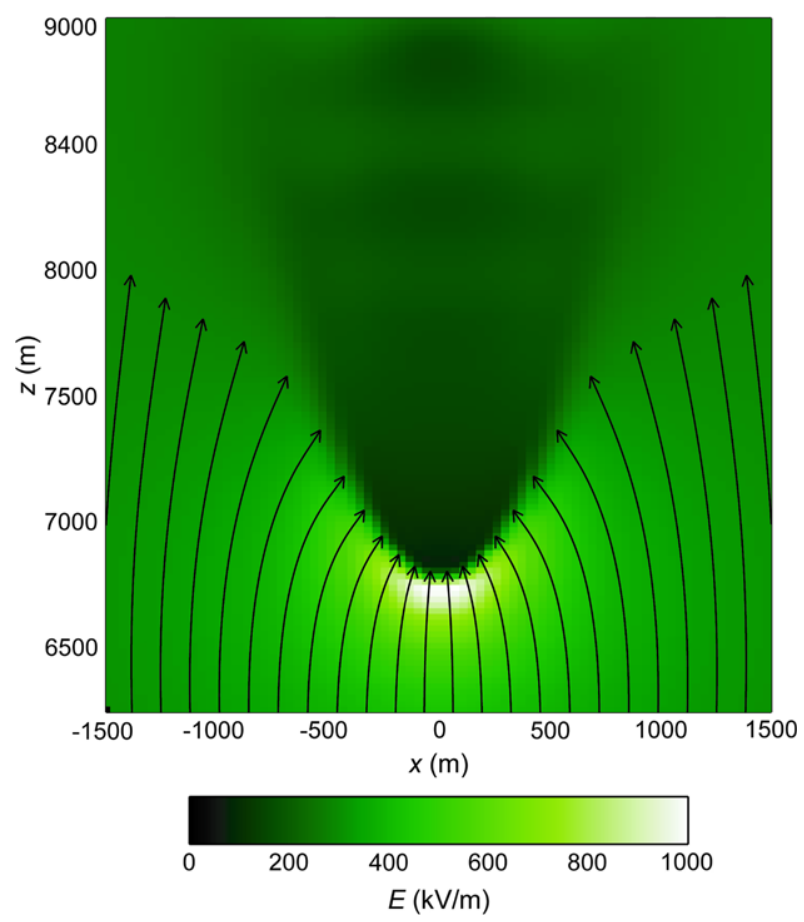

Fig. 28 Simulation of a relativistic feedback discharge inside a thundercloud. Relativistic feedback creates a propagating discharge region that moves downward in the figure. The gamma-ray emission generated by the discharge resembles the emissions recorded for TGFs. The vertical axis is height above sea-level. The color scale gives the sea-level equivalent electric field strength with black corresponding to no electric field and white the maximum electric field. The black curves with arrows are samples of the average trajectories of the runaway electrons for $E>E_{t h}$. The positive and negative charge regions are located at the top and bottom of the figure, respectively. The maximum electric field produced at the head of the discharge at this point in the simulation was $430 \mathrm{kV} / \mathrm{m}$, which corresponds to a field of $1000 \mathrm{kV} / \mathrm{m}$ at sea-level. The field at the discharge head is already 2.86 times the maximum ambient field and continues rising as the discharge propagates towards the bottom of the figure. Figure from Dwyer (2005b)

found that the leaders decelerate during the first $10-15 \mathrm{~ms}$ of the discharge. They interpreted their results as evidence for preconditioning of the air by energetic electron avalanches. TGFs are expected to be produced in high field regions, so it is plausible that TGFs are occurring in the same high field regions where lightning is initiated. As a result, addressing the causes of TGFs may give insight into the lightning initiation problem as well.

Section 4.4 summarized the different analyses of different radio emissions associated with TGFs detected by different satellites. Although these past studies provide different views into the TGF-generating process, all of these studies are consistent with the same general picture. We summarize this picture below with a list of established findings on the connection of observed TGFs and lightning processes:

- TGFs are produced by a thunderstorm process that is generally within 25 degrees of the point directly below detecting satellite. In some instances, however, this angle can be larger 45 degrees.

- TGFs are produced in an in-cloud process with equivalent peak currents and total charge moment changes that are often (but not always) strong for IC processes. However, these 
peak currents and charge moment changes are too small to produce TGFs at high altitudes via EMP or slowly varying electric fields.

- TGFs are produced in what are structurally normal IC flashes during the period when the initial negative polarity leader travels upward from the main negative charge layer to the upper positive layer. This occurs during the first 5-10 ms of the lightning flash but distinctly after the flash initiation.

- TGFs occur during the ascent of this upward leader before it reaches and expands into the upper positive charge layer. Inferred altitudes of the leader tip at the time of TGF generation have range from approximately 11 to $15 \mathrm{~km}$.

- TGFs are produced during a several ms period when the upward leader radiates significant LF pulses from fast (several tens of $\mu \mathrm{s}$ ) processes, which are possibly leader steps. However, TGFs do not appear to be specifically connected to one of these pulses.

- TGFs are produced essentially simultaneously (within $\sim 10 \mu \mathrm{s}$ ) with a slower radiation pulse ( 100-200 $\mu$ s duration) that implies a process with a net current variation that mirrors this time scale. It is possible that this is radiation directly from the energetic electrons that generate TGF gamma rays and from the associated ionization (i.e. lowenergy electrons and ions).

It should be emphasized that, with the possible exception of the AGILE $100 \mathrm{MeV}$ observation, at the time of this writing, there are no reported measurements associated with detected TGFs that support a mechanism or picture different than that described above. This is not to say that other possible mechanisms described in the literature, such as extremely high (>6000 C km) CG lightning charge moment changes (Lehtinen et al. 1999) or very high peak currents combined with extraordinarily high stroke velocities (Inan and Lehtinen 2005) can never happen. It is simply that no observed TGF appears to be associated with these mechanisms.

There remains much that we do not understand about the relationship between lightning and TGFs. What physical processes actually occur during the initial upward leader propagation in an IC lightning flash is not very well understood (Shao and Krehbiel 1996). The significance of the different time scale processes associated with TGFs, which vary from about $10 \mu \mathrm{s}$ to several $\mathrm{ms}$, is similarly poorly understood. And the observations of multiple TGFs and multiple sferics that are not always linked (Cohen et al. 2010b; Lu et al. 2011) continue to be confusing.

Perhaps the most important question is, what is different about otherwise normal IC flashes that produce TGFs? Is the TGF-generating process common and just the source altitude (Williams et al. 2006; Smith et al. 2010) dictates whether the TGF is detectable at high altitudes? Recent work suggests not (Smith et al. 2011b) but identifying a definitive signature of TGF-producing lightning flashes would give important insight into what is different about these flashes.

\subsection{Runaway Electrons and Compact Intra-Cloud Discharges}

Compact Intra-Cloud Discharges (CIDs) are the name given to the discharge within thunderclouds that results in radio emissions called narrow bipolar events (NBEs), or narrow bipolar pulses (NBPs) (Smith et al. 1999; Le Vine 1980). NBEs are the most powerful natural radio emission in the Earth environment, more intense in the VHF range than either normal intra-cloud lightning or cloud-to-ground return strokes (Nag and Rakov 2010). NBEs also appear as high amplitude sferics (wide band DC to $20 \mathrm{MHz}$ waveforms) lasting about $10-30 \mu$ s with very rapid, $\sim 1 \mu$ s, rise times. NBEs often appear as isolated events with no detectable leader activity before the main sferic pulse and with little light 
emission detectable by satellites during the discharge, as opposed to other in-cloud processes. NBEs have also been found to be associated with TGFs (Stanley et al. 2006; Lu et al. 2010). Because the NBEs show very rapid rises in the current without any apparent leader activity beforehand, several authors have investigated the possibility that NBEs are produced by the joint action of cosmic-ray extensive air showers and RREAs (see Sect. 2.5). A related study by Gurevich et al. (2003) measured initial bipolar pulses associated with lightning and found that the measurements agreed their EAS-RREA calculations (Gurevich et al. 2002). Further modeling by Gurevich and Zybin (2004) indicated that NBEs could be the result of cosmic-ray extensive air showers seeding large RREAs. Watson and Marshall (2007) used transmission line models of the current to study NBEs and suggested that runaway electrons were involved in the process. However, Dwyer et al. (2009) modeled the RF emissions from EAS-RREAs and questioned whether it could produce large enough pulses to be consistent with NBEs. Specifically, when standard ionization rates are used and the maximum avalanche multiplication factor determined by relativistic feedback is taken into account, Dwyer et al. (2009) found that the RF pulses produced by the EAS-RREA mechanism are too small to explain NBEs unless the very largest air showers are considered. As a result, at this time it is not clear if this mechanism can account for NBEs, or play a role in generating a hot channel used in some models of CIDs.

Another problem for understanding NBEs is their duration, which is sometimes $>20 \mu \mathrm{s}$. Because this time is longer than the electron attachment times for at least part of the altitude range for which NBEs occur, the total current pulse may have to come from either a hot channel that is formed or from a runaway electron production region that extends many kilometers. Neither one of these options have been shown to be viable. In particular, it has not been demonstrated how a diffuse discharge of runaway electrons, hundreds of meters across, can result in a narrow hot channel. A similar problem arises when consider lightning initiation by the EAS-RREA mechanism. Yet another challenge in explaining NBEs is the rapidly falling energy spectrum of the primary cosmic-rays. For every large RF pulse caused by a high energy cosmic ray, there should be many smaller pulses caused by slightly lower energy cosmic-rays. It is not clear if such smaller pulses with the correct intensity distribution are present. All in all, it is still possible that extensive air showers are playing some role in NBEs and CID, but further investigations, theoretical and observational, are required.

\subsection{RREA, Sprites, and Other TLEs}

It is important to mention that in the first few years after the discovery of TGFs, numerous groups investigated RREAs at high altitudes and proposed that sprites, blue jets and terrestrial gamma-ray flashes (TGF) were all manifestations of these high altitude runaway electrons avalanches (Bell et al. 1995; Gurevich et al. 1996; Lehtinen et al. 1996, 1997, 2000; Milikh and Valdivia 1999; Kutsyk and Babich 1999; Roussel-Dupré and Gurevich 1996; Roussel-Dupré et al. 1998; Taranenko and Roussel-Dupré 1996; and Yukhimuk et al. 1998a, 1998b, 1999). Although this idea was promising at the time, it is now certain that runaway electron models do not describe sprites (Pasko 2010). Nevertheless, much progress in the physics of runaway electrons at high altitudes occurred in this early work, including the effects of the geomagnetic fields on the runaway electrons (Papadopoulos et al. 1996; Papadopoulos and Valdivia 1997; Gurevich et al. 1996).

Alternatively, Chang and Price (1995) considered thermal runaway electron production in the large scale field above thunderclouds. In light of measurements of TGF-associated lightning, it can be stated that this is also not the production mechanism of observed TGFs. It is possible that some thermal runaway electron production may be occurring at the sprite 
streamer heads (Pasko et al. 1998), since a similar process is thought to occur for steamers at sea-level (e.g., Moss et al. 2006). However, such emission is not consistent with TGF observations. Indeed, a large amount of observation and modeling now supports the conclusion that sprites are a conventional streamer-type discharge, and that observed TGFs are not associated with sprites and occur deep in the atmosphere below about $20 \mathrm{~km}$.

Furthermore, the size and timing of the lightning processes associated with TGFs (reviewed in Sect. 4.4 and summarized in Sect. 5.1.3) can be used to rule out high altitude RREA as the source of most TGFs. Nevertheless, recent work by Galper and Koldashov (2007) has revisited this topic, modeling high altitude runaway electron beams. Füllekrug et al. (2010, 2011) have claimed to have identified RF signatures of energetic electron beams due to high altitude RREA in association with sprites. However, it can again be said that the observations of lightning associated with TGFs are not consistent with this explanation. Moreover, is not clear if such energetic electron beams, with extremely large fluences ejected into space, are the only explanation for the observed radio emission, as lightning flashes as complex as those that typically produce sprites (Shao and Krehbiel 1996; Lu et al. 2009) commonly produce impulsive LF radio pulses of the type reported by Füllekrug et al. $(2010,2011)$ through ordinary lightning processes.

\subsection{TGF Radiation Doses}

Even at spacecraft altitudes, TGFs are very bright. In fact, they are so bright that spacecraft designed to measure powerful x-ray and gamma-ray bursts from the sun and astrophysical sources often experience large dead-times during TGFs (Grefenstette et al. 2008; Gjesteland et al. 2010). The REAM Monte Carlo simulations of the bremsstrahlung emissions from RREA, taking into account the propagation of gamma-rays through the atmosphere, including Compton scattering, photo-electric absorption and pair production, showed that there must be about $10^{17}$ high-energy $(\mathrm{MeV})$ electrons present inside or just above the thundercloud in order to generate the number of gamma-rays measured $>600 \mathrm{~km}$ away in space (Dwyer and Smith 2005). This is a very large number of energetic electrons. Considering that they were produced in less than $1 \mathrm{~ms}$, the duration of the TGF, the fluence (number per $\mathrm{m}^{2}$ ) of the energetic electrons must have been at least ten billion times higher than the cosmic ray particles that were passing through the thundercloud. If an aircraft were struck by such a TGF at the source, the radiation dose received by individuals inside the aircraft could be significant. Dwyer et al. (2010) calculated the effective dose received by an individual inside an aircraft in the runaway electron beam that produces a TGF. The main uncertainty in the calculation is the lateral size of the TGF source region, so Dwyer et al. (2010) presented the effective dose as a function of the electron beam diameter. This is shown in Fig. 29. The horizontal lines are the maximum allowed dose for members of the public and the dose that would result in mild radiation sickness.

\section{Summary}

The newly emerging field of high-energy atmospheric physics is the study of the production of high-energy runaway electrons and their accompanying x-ray and gamma-ray emissions in our atmosphere. It has now been established that such energetic processes commonly occur in association with terrestrial thunderstorms and lightning. Because electrified clouds and lightning are known to occur in other planetary atmospheres (e.g., Jupiter and Saturn) and the physics of runaway electrons is similar in other gaseous media, it is expected that 
Fig. 29 The effective dose produced by a TGF versus the diameter of the energetic electron beam. The curve is the effective dose for a TGF event with $2 \times 10^{17}$ runaway electrons. The horizontal dashed lines show the maximum dose permitted for the general population (labeled regulatory limit), and the dose in which mild radiation sickness would occur. Figure from Dwyer et al. (2010)

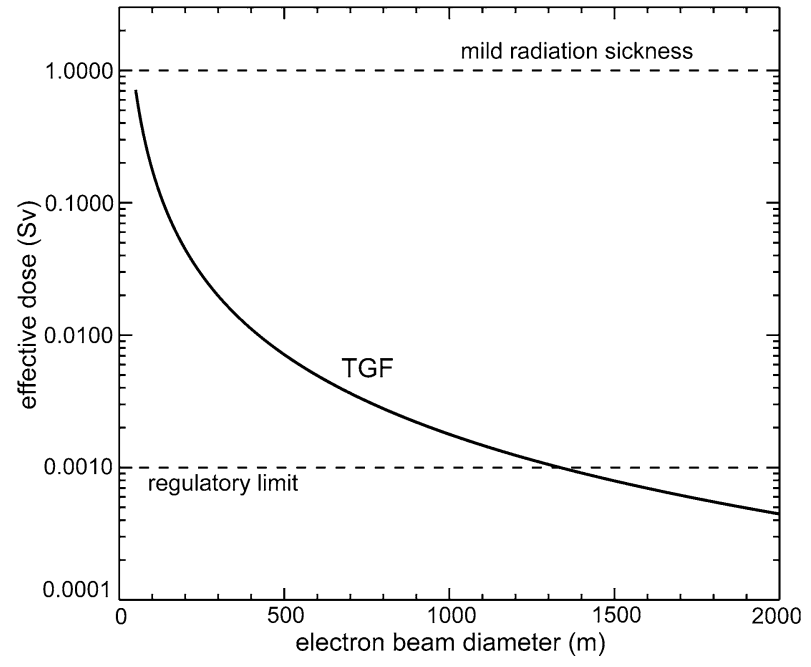

runaway electrons also will be common in other planetary atmospheres in our solar system (Dwyer et al. 2006; Roussel-Dupré et al. 2008; Yair et al. 2008). Although still under investigation, it is plausible that high-energy phenomena play important roles in atmospheric electrodynamics. In particular, high-energy atmospheric physics should be considered in the study of lightning initiation and propagation, thundercloud electrification, terrestrial gamma-ray flashes, narrow bipolar events, RF emissions from cosmic-ray air showers, and laboratory discharges.

Acknowledgements We thank Dr. Martin Uman and Dustin Hill along with members of the Geospace Physics Laboratory for their input. We also thank the reviewer for his/her valuable comments and corrections. This work has been supported in part by the NSF grants ATM-0607885, ATM-0846609, ATM-1042198, and ATM-1047588, and by DARPA grants HR0011-1-10-1-0061 and HR0011-10-1-0059.

Open Access This article is distributed under the terms of the Creative Commons Attribution License which permits any use, distribution, and reproduction in any medium, provided the original author(s) and the source are credited.

\section{References}

S. Agostinelli et al., Nucl. Instrum. Methods 506(3), 250-303 (2003)

V.V. Alexeenko, N.S. Khaerdinov, A.S. Lidvansky, V.B. Petkov, Phys. Lett. A 301, 299 (2002)

V.P. Antonova et al., Tech. Phys. 52, 1496-1501 (2007)

V.P. Antonova, L.I. Vildanova, A.V. Gurevich, K.P. Zybin, A.N. Karashtin, S.V. Kryukov, V.A. Ryabov, M.O.

Ptitsyn, A.P. Chubenko, Yu.V. Shlyugaev, A.L. Schepetov, Radiophys. Quantum Electron. 52, 9 (2009)

E.V. Appleton, E.G. Bowen, Nature 132, 965 (1933)

I.V. Arkhangelskaja, NATO Sci. Ser. 225, 377 (2006)

L.P. Babich, High-Energy Phenomena in Electric Discharges in Dense Gases (Futurepast, Arlington, 2003)

L.P. Babich, J. Exp. Theor. Phys. 98(4), 707-718 (2004)

L.P. Babich, JETP Lett. 84, 285 (2006)

L.P. Babich, Geomagn. Aeron. 47, 664 (2007)

L.P. Babich, Private communications (2010)

L.P. Babich, E.I. Bochkov, J. Exp. Theor. Phys. 112(3), 494-503 (2011)

L.P. Babich, R.A. Roussel-Dupré, J. Geophys. Res. 112, D13303 (2007)

L.P. Babich, I.M. Kutsyk, E.N. Donskoy, A.Yu. Kudryavtsev, Phys. Lett. A 245, 460-470 (1998)

L.P. Babich et al., IEEE Trans. Plasma Sci. 29(3), 430-438 (2001a) 
L.P. Babich et al., Dokl. Earth Sci. 381, 994-997 (2001b)

L.P. Babich et al., Dokl. Phys. 47, 1-4 (2002)

L.P. Babich, E.N. Donskoy, R.I. Il'kaev, I.M. Kutsyk, R.A. Roussel-Dupré, Plasma Phys. Rep. 30, 616-624 (2004a)

L.P. Babich et al., High Temp. 42, 1-10 (2004b)

L.P. Babich et al., Geomagn. Aeron. 44, 243-251 (2004c)

L.P. Babich, E.N. Donskoy, I.M. Kutsyk, R.A. Roussel-Dupré, Geophys. Res. Lett. 32, L09809 (2005)

L.P. Babich, A.Y. Kudryavtsev, A. Yu, M.L. Kudryavtseva, I.M. Kutsyk, Dokl. Earth Sci. 415, 885 (2007a)

L.P. Babich, E.I. Bochkov, I.M. Kutsyk, Geomagn. Aeron. 47, 671-675 (2007b)

L.P. Babich, E.N. Donskoy, R.A. Roussel-Dupré, Geomagn. Aeron. 47(4), 515-524 (2007c)

L.P. Babich, A.Yu. Kudryavtsev, M.L. Kudryavtseva, I.M. Kutsyk, JETP Lett. 85(10), 483-487 (2007d)

L.P. Babich, A.Y. Kudryavtsev, M.L. Kudryavtseva, I.M. Kutsyk, J. Exp. Theor. Phys. 106, 65 (2008a)

L.P. Babich, A.Yu. Kudryavtsev, M.L. Kudryavtseva, I.M. Kutsyk, J. Exp. Theor. Phys. 106, 65-76 (2008b)

L.P. Babich, A.Yu. Kudryavtsev, M.L. Kudryavtseva, I.M. Kutsyk, Geomagn. Aeron. 48, 367-377 (2008c)

L.P. Babich, A.Yu. Kudryavtsev, M.L. Kudryavtseva, I.M. Kutsyk, Geomagn. Aeron. 48, 378-391 (2008d)

L.P. Babich, E.N. Donskoy, I.M. Kutsyk, J. Exp. Theor. Phys. 107, 49 (2008e)

L.P. Babich, E.N. Donskoi, A.Yu. Kudryavtsev, M.L. Kudryavtseva, I.M. Kutsyk, AIP Conf. Proc. 1118, 52-57 (2009a)

L.P. Babich, E.I. Bochkov, I.M. Kutsyk, Geomagn. Aeron. 49(2), 232-238 (2009b)

L.P. Babich, E.I. Bochkov, E.N. Donskoi, I.M. Kutsyk, J. Geophys. Res. 115, A09317 (2010a)

L.P. Babich, E.I. Bochkov, I.M. Kutsyk, R.A. Roussel-Dupré, J. Geophys. Res. 115, A00E28 (2010b)

L.P. Babich, E.I. Bochkov, I.M. Kutsyk, J. Exp. Theor. Phys. 112, 902-909 (2011a)

L. Babich, C. Haldoupis, A. Kudryavtsev, I. Kutsyk, J. Geophys. Res. 116, A09313 (2011b)

K.I. Bakhov, L.P. Babich, I.M. Kutsyk, IEEE Trans. Plasma Sci. 28, 1254-1262 (2000)

E.M. Bazelyan, Yu.P. Raizer, Spark Discharge (CRC Press, Boca Raton, 1998)

T.F. Bell, V.P. Pasko, U.S. Inan, Geophys. Res. Lett. 22, 2127-2130 (1995)

S.A. Behnke et al., J. Geophys. Res. 110, D10207 (2005)

V.B. Berestetskii, E.M. Lifshitz, L.P. Pitaevskii, Quantum Electrodynamics (Pergamon, Oxford, 1982)

C.J. Biagi, M.A. Uman, J.D. Hill, D.M. Jordan, V.A. Rakov, J. Dwyer, J. Geophys. Res. 115, D23215 (2010)

L.S. Bratolyubova-Tsulukidze, E.A. Grachev, O.R. Grigoryan, V.E. Kunitsyn, B.M. Kuzhevskij, D.S. Lysakov, O.Yu. Nechaev, M.E. Usanova, Adv. Space Res. 34(8), 1815-1818 (2004)

M.S. Briggs, Partially ionized plasmas throughout the cosmos, in Proceedings of the 2010 Huntsville Workshop. AIP Conf. Proc., vol. 1366 (2011), p. 47

M.S. Briggs et al., J. Geophys. Res. 115(A7), A07323 (2010)

M.S. Briggs, V. Connaughton, C. Wilson-Hodge, R.D. Preece, G.J. Fishman, R.M. Kippen, P.N. Bhat, W.S. Paciesas, V.L. Chaplin, C.A. Meegan, A. von Kienlin, J. Greiner, J.R. Dwyer, D.M. Smith, Geophys. Res. Lett. 38(2), L02808 (2011)

M. Brunetti, S. Cecchini, M. Galli, G. Giovannini, A. Pagliarin, Geophys. Res. Lett. 27, 1599-1602 (2000)

S. Buitink et al., Astron. Astrophys. 467, 385-394 (2007)

S. Buitink, T. Huege, H. Falcke, D. Heck, J. Kuijpers, Astropart. Phys. 33(1), 1-12 (2010a)

S. Buitink, T. Huege, H. Falcke, J. Kuijpers, Astropart. Phys. 33(5-6), 296-306 (2010b)

B. Carlson, PhD dissertation, Stanford University (2009)

B. Carlson, N. Lehtinen, U. Inan, Geophys. Res. Lett. 34(8), L08809 (2007)

B. Carlson, N. Lehtinen, U.S. Inan, J. Geophys. Res. 113, A10307 (2008)

B. Carlson, N. Lehtinen, U. Inan, J. Geophys. Res. 114, A00E08 (2009a)

B. Carlson, N. Lehtinen, U. Inan, Coupling of thunderstorms and lightning discharges to near-earth space, in Proceedings of the Workshop. AIP Conf. Proc., vol. 1118 (2009b), pp. 84-91

B. Carlson, N. Lehtinen, U. Inan, J. Geophys. Res. 115(A10), A10324 (2010a)

B. Carlson, N. Lehtinen, U. Inan, J. Geophys. Res. 115, A00E19 (2010b)

B.E. Carlson, T. Gjesteland, N. Østgaard, J. Geophys. Res. 116, A11217 (2011)

S. Celestin, V. Pasko, J. Phys. D, Appl. Phys. 43, 315206 (2010)

S. Celestin, V. Pasko, J. Geophys. Res. 116, A03315 (2011)

R. Chang, C. Price, Geophys. Res. Lett. 22, 1117-1120 (1995)

O. Chanrion, T. Neubert, J. Comput. Phys. 227(15), 7222-7245 (2008)

O. Chanrion, T. Neubert, J. Geophys. Res. 115, A00E32 (2010)

A.B. Chen et al., J. Geophys. Res. 113, A08306 (2008)

A. Chilingarian, A. Daryan, K. Arakelyan, A. Hovhannisyan, B. Mailyan, L. Melkumyan, G. Hovsepyan, S. Chilingaryan, A. Reymers, L. Vanyan, Phys. Rev. D 82(4), 043009 (2010)

H.J. Christian et al., J. Geophys. Res. D 108, 4-1 (2003)

A.P. Chubenko, V.P. Antonova, S.Yu. Kryukov, V.V. Piskal, M.O. Ptitsyn, A.L. Shepetov, L.I. Vildanova, K.P. Zybin, A.V. Gurevich, Phys. Lett. A 275, 90-100 (2000) 
A.P. Chubenko et al., Phys. Lett. A 309, 90 (2003)

A.P. Chubenko et al., Phys. Lett. A 373(33), 2953-2958 (2009)

J. Clay, H.F. Jongen, A.J.J. Aarts, Physica 28, 801-808 (1952)

M.B. Cohen, U.S. Inan, G.J. Fishman, J. Geophys. Res. 111(D24), D24109 (2006)

M.B. Cohen, U.S. Inan, R.K. Said, M.S. Briggs, G.J. Fishman, V. Connaughton, S.A. Cummer, Geophys. Res. Lett. 37(18), L18806 (2010a)

M.B. Cohen, U.S. Inan, R.K. Said, T. Gjestland, Geophys. Res. Lett. 37(2), L02801 (2010b)

L.M. Coleman, J.R. Dwyer, Geophys. Res. Lett. 33, L11810 (2006)

A.B. Collier, T. Gjesteland, N. Østgaard, J. Geophys. Res. A 116, A10320 (2011)

J.J. Colman, R.A. Roussel-Dupré, L. Triplett, J. Geophys. Res. 115, A00E16 (2010)

V. Connaughton, M.S. Briggs, R.H. Holzworth, M.L. Hutchins, G.J. Fishman, C.A. Wilson-Hodge, V.L. Chaplin, P.N. Bhat, J. Greiner, A. von Kienlin, R.M. Kippen, C.A. Meegan, W.S. Paciesas, R.D. Preece, E. Cramer, J.R. Dwyer, D.M. Smith, J. Geophys. Res. 115(A12), A12307 (2010)

V. Cooray, L. Arevalo, M. Rahman, J. Dwyer, H. Rassoul, J. Atmos. Sol.-Terr. Phys. (2009). doi:10.1016/j.jastp.2009.07.010

V. Cooray, J. Dwyer, V. Rakov, M. Rahman, J. Atmos. Sol.-Terr. Phys. 72(11), 848-855 (2010)

S.A. Cummer, Y. Zhai, W. Hu, D.M. Smith, L.I. Lopez, M.A. Stanley, Geophys. Res. Lett. 32, L08811 (2005)

S.A. Cummer, G. Lu, M.S. Briggs, V. Connaughton, S. Xiong, G.J. Fishman, J.R. Dwyer, Geophys. Res. Lett. 38, L14810 (2011)

K. Cummins, M.J. Murphy, IEEE Trans. Electromagn. Compat. 51(3), 499-518 (2009)

N. D’Angelo, Ann. Geophys. Ser. B 5, 119-122 (1987)

A. Drozdov et al., J. Geophys. Res. 115, A00E51 (2010)

J.R. Dwyer, Geophys. Res. Lett. 30, 2055 (2003)

J.R. Dwyer, Geophys. Res. Lett. 31, L12102 (2004)

J.R. Dwyer, Sci. Am. (May issue) (2005a)

J.R. Dwyer, Geophys. Res. Lett. 32, L20808 (2005b)

J.R. Dwyer, Phys. Plasmas 14, 042901 (2007)

J.R. Dwyer, J. Geophys. Res. 113(D10), D10103 (2008)

J.R. Dwyer, in Energetic Radiation and Lightning, Lightning: Principles, Instruments and Applications, ed. by H.D. Betz, U. Schumann, P. Laroche (Springer, Berlin, 2009)

J.R. Dwyer, J. Geophys. Res. 115, A00E14 (2010)

J.R. Dwyer, J. Geophys. Res. 117, A02308 (2012)

J.R. Dwyer, L. Babich, J. Geophys. Res. 116, A09301 (2011)

J.R. Dwyer, H.K. Rassoul, J. Geophys. Res. 116, A08312 (2011)

J.R. Dwyer, D.M. Smith, Geophys. Res. Lett. 32, L22804 (2005)

J.R. Dwyer, D.M. Smith, J. Geophys. Res. 115, JD012255 (2010)

J.R. Dwyer, M.A. Uman, H.K. Rassoul, M. Al-Dayeh, E.L. Caraway, J. Jerauld, V.A. Rakov, D.M. Jordan, K.J. Rambo, V. Corbin, B. Wright, Science 299, 694-697 (2003)

J.R. Dwyer, M.A. Uman, H.K. Rassoul, V.A. Rakov, M. Al-Dayeh, E.L. Caraway, B. Wright, J. Jerauld, D.M. Jordan, K.J. Rambo, A. Chrest, C. Smyth, Geophys. Res. Lett. 31, L05119 (2004a)

J.R. Dwyer, M.A. Uman, H.K. Rassoul, V.A. Rakov, M. Al-Dayeh, E.L. Caraway, B. Wright, J. Jerauld, D.M. Jordan, K.J. Rambo, A. Chrest, C. Smyth, Geophys. Res. Lett. 31, L05118 (2004b)

J.R. Dwyer, H.K. Rassoul, Z. Saleh, M.A. Uman, J. Jerauld, J.A. Plumer, Geophys. Res. Lett. 32, L20809 (2005a)

J.R. Dwyer, M.A. Uman, H.K. Rassoul, V.A. Rakov, M. Al-Dayeh, E.L. Caraway, B. Wright, J. Jerauld, D.M. Jordan, K.J. Rambo, A. Chrest, E. Kozak, Geophys. Res. Lett. 32, L01803 (2005b)

J.R. Dwyer, L.M. Coleman, R. Lopez, Z. Saleh, D. Concha, M. Brown, H.K. Rassoul, Geophys. Res. Lett. 33, L22813 (2006)

J.R. Dwyer et al., J. Geophys. Res. 113(D23), D23207 (2008a)

J.R. Dwyer, B.W. Grefenstette, D.M. Smith, Geophys. Res. Lett. 35, L02815 (2008b)

J.R. Dwyer, M.A. Uman, H.K. Rassoul, J. Geophys. Res. 114, D09208 (2009)

J.R. Dwyer, D.M. Smith, M.A. Uman, Z. Saleh, B. Grefenstette, B. Hazelton, H.K. Rassoul, J. Geophys. Res. 115, D09206 (2010)

J.R. Dwyer, M. Schaal, H.K. Rassoul, M.A. Uman, D.M. Jordan, D. Hill, H.K. Rassoul, J. Geophys. Res. 116, D20208 (2011)

K.B. Eack, W.H. Beasley, W.D. Rust, T.C. Marshall, M. Stolzenburg, J. Geophys. Res. 101(D23), 2963729640 (1996a)

K.B. Eack, W.H. Beasley, W.D. Rust, T.C. Marshall, M. Stolzenburg, Geophys. Res. Lett. 23(21), 2915-2918 (1996b)

K.B. Eack, D.M. Suszcynsky, W.H. Beasley, R. Roussel-Dupré, E. Symbalisty, Geophys. Res. Lett. 27, 185$188(2000)$ 
M. Ender et al., in Proc. of the 31st ICRC (2009)

D. Fargion, Astrophys. J. 570(2), 909-925 (2002)

H. Feng, T.P. Li, M. Wu, M. Zha, Q.Q. Zhu, Geophys. Res. Lett. 29, 6 (2002)

G.J. Fishman et al., in Proc. GRO Science Workshop, ed. by W.N. Johnson (NASA/GSFC, Greenbelt, 1989), pp. 2-39

G.J. Fishman et al., Science 264, 1313 (1994)

G.J. Fishman et al., J. Geophys. Res. 116, A07304 (2011)

R.L. Fleischer, J. Geophys. Res. 80, 5005 (1975)

M. Füllekrug, R. Roussel-Dupré, E.M.D. Symbalisty, O. Chanrion, A. Odzimek, O. van der Velde, T. Neubert, J. Geophys. Res. 115(16), A00E09 (2010)

M. Füllekrug et al., Atmos. Chem. Phys. 11(15), 7747 (2011)

F. Fuschino et al., Geophys. Res. Lett. 38, L14806 (2011)

A.M. Galper, S.V. Koldashov, Cosm. Res. 45(5), 452-454 (2007)

E.A. Gerken, U.S. Inan, C.P. Barrington-Leigh, Geophys. Res. Lett. 27(17), 2637-2640 (2000)

T. Gjesteland, N. Østgaard, P.H. Connell, J. Stadsnes, G.J. Fishman, J. Geophys. Res. 115(3), A00E21 (2010)

T. Gjesteland, N. Østgaard, A. Collier, B.E. Carlson, M.B. Cohen, N.G. Lehtinen, J. Geophys. Res. 116, A11313 (2011)

T. Gjesteland, N. Østgaard, A.B. Collier, B.E. Carlson, C. Eyles, D.M. Smith, Geophys. Res. Lett. 39, L05102 (2012)

B.W. Grefenstette, D.M. Smith, J.R. Dwyer, G.J. Fishman, Geophys. Res. Lett. 35(6), L06802 (2008)

B.W. Grefenstette, D.M. Smith, B.J. Hazelton, L.I. Lopez, J. Geophys. Res. 114, A02314 (2009)

M.B. Greenfield et al., J. Appl. Phys. 93, 1839-1844 (2003)

E. Grove, Private communication (2011)

A.V. Gurevich, Sov. Phys. JETP 12(5), 904-912 (1961)

A.V. Gurevich, G.M. Milikh, Phys. Lett. A 262, 457-463 (1999)

A.V. Gurevich, K.P. Zybin, Phys. Usp. 44, 1119 (2001)

A.V. Gurevich, K.P. Zybin, Phys. Lett. A 329(4-5), 341-347 (2004)

A.V. Gurevich, K.P. Zybin, Phys. Today 58(5), 37-43 (2005)

A.V. Gurevich, G.M. Milikh, R.A. Roussel-Dupré, Phys. Lett. A 165, 463 (1992)

A.V. Gurevich, G.M. Milikh, R.A. Roussel-Dupré, Phys. Lett. A 187, 197-203 (1994)

A.V. Gurevich, J.A. Valdivia, G.M. Milikh, K. Papadopoulos, Radio Sci. 31, 1541-1554 (1996)

A.V. Gurevich, G.M. Milikh, J.A. Valdivia, Phys. Lett. A 231, 402-408 (1997)

A.V. Gurevich, K.P. Zybin, R.A. Roussel-Dupré, Phys. Lett. A 237, 240-246 (1998)

A.V. Gurevich, K.P. Zybin, R.A. Roussel-Dupré, Phys. Lett. A 254, $79-87$ (1999)

A.V. Gurevich, H.C. Carlson, Yu.V. Medvedev, K.P. Zybin, Phys. Lett. A 275, 101-108 (2000)

A.V. Gurevich et al., Phys. Lett. A 260, 269-278 (2001a)

A.V. Gurevich, H.C. Carlson, Yu.V. Medvedev, K.P. Zybin, Phys. Lett. A 282, 180-185 (2001b)

A.V. Gurevich, L.M. Duncan, Yu.V. Medvedev, K.P. Zybin, Phys. Lett. A 301, 320-326 (2002)

A.V. Gurevich, L.M. Duncan, A.N. Karashtin, K.P. Zybin, Phys. Lett. A 312, 228-237 (2003)

A.V. Gurevich, Y.V. Medvedev, K.P. Zybin, Phys. Lett. A 321(3), 179-184 (2004a)

A.V. Gurevich, Yu.V. Medvedev, K.P. Zybin, Phys. Lett. A 329, 348-361 (2004b)

A.V. Gurevich et al., Phys. Lett. A 325, 389-402 (2004c)

A.V. Gurevich, K.P. Zybin, Yu.V. Medvedev, Phys. Lett. A 349, 331-339 (2006)

A.V. Gurevich, K.P. Zybin, Yu.V. Medvedev, Phys. Lett. A 361, 119-125 (2007)

A.V. Gurevich et al., Phys. Lett. A 373, 3550-3553 (2009a)

A.V. Gurevich et al., Phys. Usp. 735 (2009b)

A.V. Gurevich et al., Phys. Lett. A 375, 1619-1624 (2011a)

A.V. Gurevich et al., Phys. Lett. A 375, 2845-2849 (2011b)

E.C. Halliday, Proc. Camb. Philol. Soc. 30, 206-215 (1934)

E.C. Halliday, Phys. Rev. 60, 101-106 (1941)

B.J. Hazelton, PhD dissertation, University of California, Santa Cruz (2009)

B.J. Hazelton, B.W. Grefenstette, D.M. Smith, J.R. Dwyer, X.-M. Shao, S.A. Cummer, T. Chronis, E.H. Lay, R.H. Holzworth, Geophys. Res. Lett. 36, L01108 (2009)

R.D. Hill, J. Geophys. Res. 68, 6261-6266 (1963)

J.D. Hill, M.A. Uman, D.M. Jordan, J. Geophys. Res. 116, D16117 (2011)

J.D. Hill, M.A. Uman, D.M. Jordan, J.R. Dwyer, H.K. Rassoul, J. Geophys. Res. 117, D03118 (2012)

A.M. Hillas, Cosmic Rays (Pergamon, Oxford, 1972)

J. Howard, M.A. Uman, J.R. Dwyer, D. Hill, C. Biagi, Z. Saleh, J. Jerauld, H.K. Rassoul, Geophys. Res. Lett. 35(13), L13817 (2008)

J. Howard, M. Uman, C. Biagi, D. Hill, J. Jerauld, V.A. Rakov, J. Dwyer, Z. Saleh, H. Rassoul, J. Geophys. Res. 115, D06204 (2010) 
T. Huege, H. Falcke, Astropart. Phys. 24, 116-136 (2005)

U.S. Inan, Science 307, 1054-1055 (2005)

U.S. Inan, N.G. Lehtinen, Geophys. Res. Lett. 32, L19818 (2005)

U.S. Inan, S.C. Reising, G.J. Fishman, J.M. Horack, Geophys. Res. Lett. 23, 1017-1020 (1996)

U.S. Inan, M.B. Cohen, R.K. Said, D.M. Smith, L.I. Lopez, Geophys. Res. Lett. 33(18), L18802 (2006)

U.B. Jayanthi et al., in 29th International Cosmic Ray Conference Pune (2005), pp. 101-104

J.V. Jelley, J.H. Fruin, N.A. Porter, T.C. Weekes, F.G. Smith, R.A. Porter, Nature 205, 327 (1965)

F.D. Kahn, I. Lerche, Proc. R. Soc. Lond. Ser. A 289, 206 (1966)

P.K. Kaw, G.M. Milikh, A.S. Sharma, P.N. Guzdar, K. Papadopoulos, Phys. Plasmas 8, 4954-4959 (2001)

N.A. Kelley, A. Lowell, D.M. Smith, J.R. Dwyer, S.A. Cummer, G. Lu, R. Blakeslee, Abstract \#AE11 A-0332 presented at 2010 Fall Meeting, AGU, San Francisco, CA., 13-17 December (2010)

R.W. Klebesadel, I.B. Strong, R.A. Olson, Astrophys. J. 182, L85 (1973)

H.W. Koch, J.W. Motz, Rev. Mod. Phys. 31(4), 920 (1950)

P.E. Krider, Science 31, 299(5607), 669-670 (2003)

I.M. Kutsyk, L.P. Babich, Phys. Lett. A 253(1-2), 75-82 (1999)

B.M. Kuzhevskii, Mosc. Univ. Phys. Bull., Ser. 3(5), 14-16 (2004)

V. Kuznetsov, Y. Ružín, V.M. Sinelnikov, Kosm. Nauka Teh. 17, 12 (2011)

C. Labanti et al., Nucl. Instrum. Methods Phys. Res. A 598, 470 (2009)

D.M. Le Vine, J. Geophys. Res. 85, 4091-4095 (1980)

F. Lefeuvre et al., Space Sci. Rev. 137, 301 (2008)

N.G. Lehtinen, M. Walt, U.S. Inan, T.F. Bell, V.P. Pasko, Geophys. Res. Lett. 23, 2645-2648 (1996)

N.G. Lehtinen, T.F. Bell, V.P. Pasko, U.S. Inan, Geophys. Res. Lett. 24, 2639-2642 (1997)

N.G. Lehtinen, T.F. Bell, U.S. Inan, J. Geophys. Res. 104, 24699-24712 (1999)

N.G. Lehtinen, U.S. Inan, T.F. Bell, Geophys. Res. Lett. 27, 1095-1098 (2000)

N.G. Lehtinen, U.S. Inan, T.F. Bell, J. Geophys. Res. 106, 28841-28856 (2001)

C. Li, U. Ebert, W. Hundsdorfer, J. Phys. D, Appl. Phys. 42, 202003 (2009)

L.M. Libby, H.R. Lukens, J. Geophys. Res. 78, 5902 (1973)

A.S. Lidvansky, J. Phys. G: Nucl. Part. Phys. 29925-29937 (2003)

R.P. Lin et al., Sol. Phys. 210, 3 (2002)

G. Lu, S.A. Cummer, J. Li, F. Han, R.J. Blakeslee, H.J. Christian, Geophys. Res. Lett. 36, L15805 (2009)

G. Lu, R.J. Blakeslee, J. Li, D.M. Smith, X.-M. Shao, E.W. McCaul, D.E. Buechler, H.J. Christian, J.M. Hall, S.A. Cummer, Geophys. Res. Lett. 37(11), L11806 (2010)

G. Lu, S.A. Cummer, J. Li, F. Han, D.M. Smith, B.W. Grefenstette, J. Geophys. Res. 116(A3), A03316 (2011)

J.L. Lundberg, R.M. Millan, K. Eack, J. Geophys. Res. 116, D21305 (2011)

D.R. MacGorman, W.D. Rust, The Electrical Nature of Storms (Oxford University Press, New York, 1998)

W.A. Macky, Proc. Camb. Philos. Soc. 30, 70-73 (1934)

V. March, J. Montanyà, Geophys. Res. Lett. 37(19), L19801 (2010)

V. March, J. Montanyà, Geophys. Res. Lett. 38(4), L04803 (2011)

M. Marisaldi et al., J. Geophys. Res. 115, A00E13 (2010a)

M. Marisaldi et al., Phys. Rev. Lett. 105, 128501 (2010b)

T. Marshall, M. McCarthy, W. Rust, J. Geophys. Res. 100(D4), 7097-7103 (1995)

T.C. Marshall et al., Geophys. Res. Lett. 32, L03813 (2005)

I.M. Martin, M.A. Alves, J. Geophys. Res. 115, A00E11 (2010)

M. McCarthy, G.K. Parks, Geophys. Res. Lett. 12, 393-396 (1985)

M.P. McCarthy, G.K. Parks, J. Geophys. Res. 97, 5857-5864 (1992)

C. Meegan et al., Astrophys. J. 702, 791 (2009)

R.R.S. De Menodonça, J.-P. Raulin, F.C.P. Bertoni, E. Echer, V.S. Makhmutov, G. Fernandez, J. Atmos. Sol.-Terr. Phys. 73, 1410 (2011)

G. Milikh, R. Roussel-Dupré, J. Geophys. Res. 115, A00E60 (2010)

G. Milikh, R. Roussel-Dupré, J. Geophys. Res. 116, A08313 (2011)

G. Milikh, J.A. Valdivia, Geophys. Res. Lett. 26, 525-528 (1999)

G.M. Milikh, P.N. Guzdar, A.S. Sharma, J. Geophys. Res. 110, A02308 (2005)

C.B. Moore, K.B. Eack, G.D. Aulich, W. Rison, Geophys. Res. Lett. 28, 2141-2144 (2001)

G.D. Moss, V.P. Pasko, N. Liu, G. Veronis, J. Geophys. Res. 111(A2), A02307 (2006)

Y. Muraki et al., Phys. Rev. D 69, 123010 (2004)

A. Nag, V.A. Rakov, J. Geophys. Res. 115, D20102 (2010)

R.J. Nemiroff, J.T. Bonnell, J.P. Norris, J. Geophys. Res. 102, 9659-9665 (1997)

T. Neubert, Coupling of thunderstorms and lightning discharges to near-Earth space, in AIP Conf. Proc. 1118, (2009), p. 8

C.V. Nguyen, A.P.J. van Deursen, U. Ebert, J. Phys. D, Appl. Phys. 41, 234012 (2008) 
C.V. Nguyen, A.P.J. van Deursen, E.J.M. van Heesch, G.J.J. Winands, A.J.M. Pemen, J. Phys. D, Appl. Phys. 43, 025202 (2010)

N. Østgaard, T. Gjesteland, J. Stadsnes, P.H. Connell, B. Carlson, J. Geophys. Res. 113(A2), A02307 (2008)

G.S. Paiva, J. Appl. Phys. 105(8), 083301 (2009)

G.S. Paiva, A.C. Pavão, C.C. Bastos, J. Geophys. Res. 114(D3), D03205 (2009)

G.S. Paiva, C.C. Bastos, A.C. Pavão, J. Geophys. Res. 116, D07205 (2011)

G.K. Parks, B.H. Mauk, R. Spiger, J. Chin, Geophys. Res. Lett. 8, 1176-1179 (1981)

V.P. Pasko, Plasma Sources Sci. Technol. 16, S13-S29 (2007)

V.P. Pasko, J. Geophys. Res. 115, A00E35 (2010)

V.P. Pasko, U.S. Inan, T.F. Bell, Geophys. Res. Lett. 25, 2123-2126 (1998)

K. Papadopoulos, J. Valdivia, Geophys. Res. Lett. 24, 2643-2644 (1997)

K. Papadopoulos, G. Milikh, J. Valdivia, Geophys. Res. Lett. 23, 2283-2284 (1996)

D. Petersen, M. Bailey, W.H. Beasley, J. Hallett, J. Geophys. Res. 113, D17205 (2008)

M. Rahman et al., Geophys. Res. Lett. 35, L06805 (2008)

V.A. Rakov, M.A. Uman, Lightning Physics and Effects (Cambridge University Press, Cambridge, 2003)

H. Raether, Electron Avalanches and Breakdown in Gases (Butterworth, Stoneham, 1964)

W. Rison, R.J. Thomas, P.R. Krehbiel, T. Hamlin, J. Harlin, Geophys. Res. Lett. 26, 3573-3576 (1999)

C.J. Rodger, J.B. Brundell, R.H. Holzworth, E.H. Lay, in Coupling of Thunderstorms and Lightning Discharges to Near-Earth Space, ed. by N.B. Crosby, T.-Y. Huang, M.J. Rycroft. AIP Conf. Proc. vol. 1118, (2009), pp. 15-20

R. Roussel-Dupré, A.V. Gurevich, J. Geophys. Res. 101(A2), 2297-2312 (1996)

R.A. Roussel-Dupré, A.V. Gurevich, T. Tunnell, G.M. Milikh, Phys. Rev. E 49, 2257-2271 (1994)

R. Roussel-Dupré, E. Symbalisty, Y. Taranenko, V. Yukhimuk, J. Atmos. Sol.-Terr. Phys. 60, 917-940 (1998)

R. Roussel-Dupré, J.J. Colman, E. Symbalisty, D. Sentman, V.P. Pasko, Space Sci. Rev. 137, 51-82 (2008)

D.E. Rowland, A.T. Weatherwax, J.H. Klenzing, J. Hill, American Geophysical Union, Fall Meeting 2009, abstract \#SM42A-07 (2009)

Z. Saleh, J. Dwyer, J. Howard, M. Uman, M. Bakhtiari, D. Concha, M. Stapleton, D. Hill, C. Biagi, H. Rassoul, J. Geophys. Res. 114, D17 (2009)

N.M. Salikhov et al., in 32nd International Cosmic Ray Conference, Beijing (2011)

A.E. Schonland, Proc. R. Soc. Lond. A 130, 37-63 (1930)

B.F.J. Schonland, J.P.T. Viljoen, Proc. R. Soc. A 140, 314-333 (1933)

K.F. Sergeichev, I.A. Sychev, Geomagn. Aeron. 42, 523-532 (2002)

G.N. Shah, H. Razdan, C.L. Bhat, Q.M. Ali, Nature 313, 773 (1985)

X.-M. Shao, P.R. Krehbiel, J. Geophys. Res. 101(26), 641-668 (1996)

X.-M. Shao, T. Hamlin, D.M. Smith, J. Geophys. Res. 115, A00E30 (2010)

G.E. Shaw, J. Geophys. Res. 72, 4623-4626 (1967)

A. Shyam, T.C. Kaushik, J. Geophys. Res. 104, 6867 (1999)

S.V. Sizykh, High Temp. 31(1), 1-6 (1993)

D.M. Smith, J. Appl. Phys. 109(2), 026101 (2011)

D.A. Smith, X.M. Shao, D.N. Holden, C.T. Rhodes, M. Brook, P.R. Krehbiel, M. Stanley, W. Rison, R.J. Thomas, J. Geophys. Res. 104, 4189-4212 (1999)

D.M. Smith et al., Sol. Phys. 210, 33 (2002)

D.M. Smith, L.I. Lopez, R.P. Lin, C.P. Barrington-Leigh, Science 307, 1085 (2005)

D.M. Smith, B.J. Hazelton, B.W. Grefenstette, J.R. Dwyer, R.H. Holzworth, E.H. Lay, J. Geophys. Res. A 115, A00E49 (2010)

D.M. Smith et al., J. Geophys. Res. 116, D20124 (2011a)

D.M. Smith, J.R. Dwyer, B.J. Hazelton, B.W. Grefenstette, G.F.M. Martinez-McKinney, Z.Y. Zhang, A.W. Lowell, N.A. Kelley, M.E. Splitt, S.M. Lazarus, W. Ulrich, M. Schaal, Z.H. Saleh, E. Cramer, H.K. Rassoul, S.A. Cummer, G. Lu, R.J. Blakeslee, Geophys. Res. Lett. 38(8), L08807 (2011b)

M.E. Splitt, S.M. Lazarus, D. Barnes, J.R. Dwyer, H.K. Rassoul, D.M. Smith, B.J. Hazelton, B.W. Grefenstette, J. Geophys. Res. 115, A00E38 (2010)

R. Solomon, V. Schroeder, M.B. Baker, Q. J. R. Meteorol. Soc. 127, 2683-2704 (2001)

R. Solomon, C. Adamo, M. Baker, C. R. Phys. 3, 1325-1333 (2002)

M. Stanley et al., Geophys. Res. Lett. 33(6), L06803 (2006)

M. Stolzenburg, T.C. Marshall, W.D. Rust, E. Bruning, D.R. MacGorman, T. Hamlin, Geophys. Res. Lett. 34, L04804 (2007)

D.M. Suszcynsky, R. Roussel-Dupré, G. Shaw, J. Geophys. Res. 101(23), 505 (1996)

E.M. Symbalisty, R.A. Roussel-Dupré, V.A. Yukhimuk, IEEE Trans. Plasma Sci. 26, 1575-1582 (1998)

Y. Taranenko, R. Roussel-Dupré, Geophys. Res. Lett. 23, 571-574 (1996)

M. Tavani et al., Astron. Astrophys. 502, 995 (2009)

M. Tavani et al., Phys. Rev. Lett. 106, 018501 (2011) 
H.E. Tierney, R.A. Roussel-Dupré, E.M.D. Symbalisty, W.H. Beasley, J. Geophys. Res. 110(D12), D12109 (2005)

D.R. Tompkins, Phys. Rev. D 10, 136-145 (1974)

T. Torii, M. Takeishi, T. Hosono, J. Geophys. Res. D 107, 4324 (2002)

T. Torii, T. Nishijima, Z.-I. Kawasaki, T. Sugita, Geophys. Res. Lett. 31, L05113 (2004)

T. Torii, T. Sugita, Y. Muraki, in Proc. 30th Int. Cosmic Ray Conf., ed. by R. Caballero et al. (2008), p. 677

T. Torii, T. Sugita, S. Tanabe, Y. Kimura, M. Kamogawa, K. Yajima, H. Yasuda, Geophys. Res. Lett. 36, L13804 (2009)

V.Y. Trakhtengerts et al., Phys. Plasmas 9, 2762-2766 (2002)

V.Y. Trakhtengerts et al., Phys. Plasmas 10, 3290-3296 (2003)

H. Tsuchiya et al., Phys. Rev. Lett. 99, 165002 (2007)

H. Tsuchiya et al., Phys. Rev. Lett. 102, 255003 (2009)

H. Tsuchiya et al., J. Geophys. Res. D 116, D09113 (2011)

M.A. Uman, Lightning (Dover, New York, 1984)

S.S. Watson, T.C. Marshall, Geophys. Res. Lett. 34, L04816 (2007)

D.P. Whitmire, Lett. Nuovo Cimento 26(16), 497-501 (1979)

E. Williams et al., J. Geophys. Res. D 111, D16209 (2006)

E.R. Williams, J. Geophys. Res. 115, A00E50 (2010)

C.T.R. Wilson, Proc. Camb. Philol. Soc. 22, 534-538 (1925)

Y. Yair, G. Fischer, F. Simões, N. Renno, P. Zarka, Space Sci. Rev. 137, 1-4 (2008)

S. Yoshida, T. Morimoto, T. Ushio, Z.-I. Kawasaki, T. Torii, D. Wang, N. Takagi, T. Watanabe, Geophys. Res. Lett. 35, L10804 (2008)

K. Yoshida et al., Trans. Jpn. Soc. Aeron. Space Sci. 8, Tm_7 (2010)

V. Yukhimuk, R.A. Roussel-Dupré, E.M.D. Symbalisty, Y. Taranenko, J. Geophys. Res. 103, 11473-11482 (1998a)

V. Yukhimuk, R.A. Roussel-Dupré, E.M.D. Symbalisty, Geophys. Res. Lett. 25, 3289-3292 (1998b)

V. Yukhimuk, R.A. Roussel-Dupré, E.M.D. Symbalisty, Geophys. Res. Lett. 26, 679-682 (1999) 